\title{
FINAL TECHNICAL REPORT ON
}

\section{CARBONIC ACID PRETREATMENT OF BIOMASS \\ DOE CONTRACT DE-FC36-01G011070, A000}

\author{
May 31, 2003
}

Covering the period from 8-1-2001 to 2-28-2003

$\begin{array}{ll}\text { Principal Investigator: } & \text { G. Peter van Walsum } \\ \text { Graduate Research Assistants: } & \begin{array}{l}\text { Kemantha Jayawardhana } \\ \text { Damon Yourchisin } \\ \text { Robert McWilliams } \\ \text { John Lam }\end{array} \\ & \begin{array}{l}\text { Connaly Miller } \\ \text { Lauren Finch }\end{array} \\ \text { Undergraduate Research Assistants: } & \text { Vanessa Castleberry }\end{array}$

Department of Environmental Studies

Baylor University

P.O. Box 97266

Waco, Texas 76798-7266

254-710-3405 
GPeter_van_Walsum@Baylor.edu 


\section{EXECUTIVE SUMMARY}

This project sought to address six objectives, outlined below. The objectives were met through the completion of ten tasks.

1) Solidify the theoretical understanding of the binary $\mathrm{CO}_{2} / \mathrm{H}_{2} \mathrm{O}$ system at reaction temperatures and pressures. The thermodynamics of $\mathrm{pH}$ prediction have been improved to include a more rigorous treatment of non-ideal gas phases. However it was found that experimental attempts to confirm theoretical $\mathrm{pH}$ predictions were still off by a factor of about $1.8 \mathrm{pH}$ units. Arrhenius experiments were carried out and the activation energy for carbonic acid appears to be substantially similar to sulfuric acid. Titration experiments

have not yet confirmed or quantified the buffering or acid suppression effects of carbonic acid on biomass.

2) Modify the carbonic acid pretreatment severity function to include the effect of endogenous acid formation and carbonate buffering, if necessary. It was found that the existing severity functions serve adequately to account for endogenous acid production and carbonate effects.

3) Quantify the production of soluble carbohydrates at different reaction conditions and severity. Results show that carbonic acid has little effect on increasing soluble carbohydrate concentrations for pretreated aspen wood, compared to pretreatment with water alone. This appears to be connected to the release of endogenous acids by the substrate. A less acidic substrate such as corn stover would derive benefit from the use of carbonic acid.

4) Quantify the production of microbial inhibitors at selected reaction conditions and severity. It was found that the release of inhibitors was correlated to reaction severity and that carbonic acid did not appear to increase or decrease inhibition compared to pretreatment with water alone.

5) Assess the reactivity to enzymatic hydrolysis of material pretreated at selected reaction conditions and severity. Enzymatic hydrolysis rates increased with severity, but no advantage was detected for the use of carbonic acid compared to water alone.

6) Determine optimal conditions for carbonic acid pretreatment of aspen wood. Optimal severities appeared to be in the mid range tested. ASPEN-Plus modeling and economic analysis of the process indicate that the process could be cost competitive with sulfuric acid if the concentration of solids in the pretreatment is maintained very high ( $50 \%)$. Lower solids concentrations result in larger reactors that become expensive to construct for high pressure applications. 


\section{TABLE OF CONTENTS}

Project Summary 1

Task 1 a) Determine accuracy of theoretical pH predictions 4

Task 1 b) Arrhenius equation for $\mathrm{H}_{2} \mathrm{CO}_{3} \quad 11$

Task 1 c) Determine buffering capacity of $\mathrm{H}_{2} \mathrm{CO}_{3} \quad 14$

Task 2) Perform laboratory experiments on pretreatment of raw biomass 21

Task 3) Compare the results between hydrolysis of purified xylan and raw biomass 38

Task 4) Test the performance of larger scale reactor 43

Task 5) Generate larger samples at selected reaction conditions 47

Task 6) In vitro determination of microbial inhibition 50

Task 7) Enzyme digestibility of pretreated solids 57

Tasks 8 +9) Aspen Modeling and Optimum pretreatment conditions 64

Task 10) Documentation 95

$\begin{array}{ll}\text { Appendices } & 96\end{array}$ 


\section{PROJECT SUMMARY}

Task 1a: Xylan and Xylose were hydrolysed in $1 \% \mathrm{H}_{2} \mathrm{SO}_{4}$ at $121{ }^{\circ} \mathrm{C}$ for varying reaction times. Samples were analyzed with high performance anion exchange (HPAE) and an ultraviolet spectrophotometer. Peak areas for xylan oligomers were integrated by completing a mass balance on samples of varying degrees of hydrolysis. This yielded an appropriate calibration for peaks representing oligomer concentrations, and confirmed theoretical expectations that the area of oligomer peaks was proportional to the molar concentration of the oligomer species. The effect of pressure on hydrolysis was tested by superpressurizing reactors with nitrogen - no pressure effect on hydrolysis was detected.

Experiments were conducted to confirm $\mathrm{pH}$ predictions of $\mathrm{CO}_{2}$ by comparing results to dilute $\mathrm{H}_{2} \mathrm{SO}_{4}$. Hydrolysis was done by subjecting a $1 \mathrm{~g} / \mathrm{L}$ xylan solution to $\mathrm{H}_{2} \mathrm{SO}_{4}$ and/or $\mathrm{CO}_{2}$ or $\mathrm{N}_{2}$ at $190^{\circ} \mathrm{C}$ or $200^{\circ} \mathrm{C}$ for 16 minutes.

Task 1b Experiments were conducted to develop an Arrhenius rate constant for sulfuric acid and $\mathrm{CO}_{2}$. These experiments consisted of hydrolyzing xylan at varying temperatures and initial pressures of $\mathrm{CO}_{2}$, while maintaining a constant $\mathrm{pH}$ of 3.4. Comparative results were generated using $\mathrm{H}_{2} \mathrm{SO}_{4}$. To characterize the species in the hydrolysate, samples were analyzed by $\mathrm{pH}, \mathrm{HPAE}$ and UV absorbency in a spectrophotometer.

Task 1c: Repeat experiments of aspen samples were hydrolyzed and titrated against a standardized $\mathrm{NaOH}$ solution to determine molar concentration of acid species in the hydrolysate. In some experiments it appeared that the acid concentration of hydrolysate of reactions with $\mathrm{CO}_{2}$ was significantly lower than the hydrolysate of reactions of wood and water alone. This confirms earlier work that consistently measured a higher $\mathrm{pH}$ in carbonic acid pretreated hydrolysates, compared to water-only pretreatment. However, in other titration experiments, the opposite result was observed, with the carbonic acid system having more accumulated acids present.

Task 2: A data set of pretreatment results on aspen using carbonic acid had been completed prior to the start of this project. Experiments continued to increase the reliability of the results and to achieve uniform performance between laboratory personnel. These objectives were achieved and reduced the scatter and uncertainty in the results.

Experiments were also carried out to determine whether the rate of mass transfer of $\mathrm{CO}_{2}$ into or out of solution exerts an effect on the rate of hydrolysis. It was found that extra $\mathrm{CO}_{2}$ could be dissolved into solution by allowing more time for the dissolution to occur, but that when the reactor was heated up to reaction temperatures there was no apparent effect on hydrolysis rates or extent.

Task 3: It was found that the severity function developed by Overend and Chornet adequately described the action of time and temperature on the pretreatment of aspen wood but not pure xylan. For aspen wood, no significant difference was detected between carbonic acid and water systems, thus the effect of the carbonic acid was negligible and did not need to be incorporated into the severity function. On xylan, however, it was necessary to take into account the action of the carbonic acid, and it was found that the severity function proposed by van Walsum did so adequately. Published literature results for the dilute acid pretreatment of softwood were used for comparing the fit of the severity function and the combined severity function for lower $\mathrm{pH}$ systems. It was found that the literature data had considerable 
scatter, but that the combined severity function did appear to offer a more predictive capability than the regular severity function, which does not take into account the $\mathrm{pH}$ of the system.

Task 4: Preliminary experiments using a $150 \mathrm{~mL}$ reactor were conducted using water and a range of $\mathrm{CO}_{2}$ pressures and reaction temperatures to evaluate the reactor performance and to determine when the pressure (and hence temperature) inside the reactor reaches steady-state. This revealed optimal reaction conditions and will minimize variation between experimental results generated from small $(15 \mathrm{~mL})$ and larger $(150 \mathrm{~mL})$ reactors. The data also provided an accurate determination of the time required for the reactor to reach reaction temperature.

Task 5: The newly constructed $150 \mathrm{~mL}$ reactor was used in reactions that replicated the conditions of the $15 \mathrm{~mL}$ reactor. 1.0 grams of aspen wood, $80 \mathrm{ml}$ of de-ionized water were reacted with and without $\mathrm{CO}_{2}$ at 800 psig. Reaction temperatures were $180^{\circ} \mathrm{C}, 200^{\circ} \mathrm{C}$, and $220^{\circ} \mathrm{C}$ with reactions times of 8,16 , and 32 minutes. The reactor was preheated in a sand bath set to a temperature $40^{\circ} \mathrm{C}$ above reaction temperature for two minutes. This allowed the reactor to quickly reach reaction temperature, as determined and reported in the previous progress report. The $150 \mathrm{ml}$ reactor successfully delivered the expected 10 -fold increase in hydrolysate compared to the $15 \mathrm{ml}$ reactor. Results of the $\mathrm{pH}$ and $\mathrm{UV}$ analysis of the hydrolysate were consistent with those yielded by the $15 \mathrm{ml}$ reactor.

Task 6: Inhibition tests measured the rate of sugar consumption by Saccharomyces cerevisiae growing in batch culture of hydrolysate. It was found that inhibition of the yeast culture increased with severity of pretreatment above a mid level severity. Below this severity, little to no inhibition was observed. No difference was observed between the inhibition of hydrolysates prepared either with or without the presence of $\mathrm{CO}_{2}$. To conduct the experiments, serum vials were charged with $21 \mathrm{~g} / \mathrm{L}$ of sterile growth medium containing $20 \mathrm{ml}$ of pretreatment hydrolysate. The vials were inoculated with $0.2 \mathrm{ml}$ of freshly grown cell broth and incubated. Glucose concentrations over time were determined via glucose assay (Infinity Glucose Reagent) and the HPAE when available.

Task 7: Enzyme digestibility tests measured enzymatic hydrolysis rates of pretreated solids by cellulase enzymes (Novozyme 188 and Iogen cellulase). It was found that more severe pretreatments enhanced enzymatic digestibility. The addition of pressurized $\mathrm{CO}_{2}$ to the pretreatment system did not significantly increase enzymatic hydrolysis rates compared to water-alone pretreatment. To conduct the experiments, $\mathrm{s}$ erum vials were charged with a $\mathrm{pH}$ 5.0 buffer, preservative, enzyme and pretreated solid sample estimated to have $2 \mathrm{~g} / \mathrm{L}$ cellulose (calculated from dry weight of the solid residue) and incubated in a $40^{\circ} \mathrm{C}$ shaker bath. Glucose concentrations over time were determined via glucose assay and the HPAE when available.

Tasks 8+ 9: Pretreatment costs for carbonic acid pretreatment are driven by the high cost of pretreatment reactors capable of containing the pressures used. This makes the cost of the carbonic acid system highly sensitive to reactor volume and thus the concentration of solids in the reactor. The cost of the reactor can be reduced by raising the concentration of the solids in the pretreatment reactor, which in turn reduces the size of the pretreatment reactor and thus diminishes the cost differential between dilute acid and carbonic acid equipment costs. If the solids concentration is put very high, and equal to that used in the NREL model, equipment costs become comparable to those for dilute sulfuric acid pretreatment. Cost of compressing $\mathrm{CO}_{2}$ is relatively low compared to the equipment cost for the high pressure reactor vessel. 
About $50 \%$ of the total operating cost is due to the heat demand of the process. This is unavoidable due to difficulties in process heat recovery.

Unless the concentration of solids in the pretreatment reactor can be increased, use of carbonic acid for pretreatment proved to be more expensive than using sulfuric acid. This is mainly due to the use of high pressures such as 2000 psi in the pretreatment reactor.

Task 10: Work from this project has been presented at four international meetings: the ACS annual meeting in Orlando Florida, April $7-11$, 2002, the $24^{\text {th }}$ Symposium on Biotechnology for Production of Fuels and Chemicals in Gatlinburg, TN, April 28 - May 1, 2002, the 12th European Conference and Technology Exhibition on Biomass for Energy, Industry and Climate Protection in Amsterdam, NL, 17-21 June 2002, and the $25^{\text {th }}$ Symposium on Biotechnology for Production of Fuels and Chemicals in Breckenridge, CO, May 3- 7, 2003. A brief paper was published in the proceedings of the Amsterdam conference and papers were submitted for peer-reviewed publication in the conference proceedings of the 25th symposium. 


\title{
TASK 1a) Determine accuracy of theoretical pH predictions
}

\author{
Vanessa Castleberry, G. Peter van Walsum
}

\begin{abstract}
Summary:
Xylan and Xylose were hydrolysed in $1 \% \mathrm{H}_{2} \mathrm{SO}_{4}$ at $121{ }^{\circ} \mathrm{C}$ for varying reaction times. Samples were analyzed with high performance anion exchange (HPAE) and an ultra-violet spectrophotometer. Peak areas for xylan oligomers were integrated by completing a mass balance on samples of varying degrees of hydrolysis. This yielded an appropriate calibration for peaks representing oligomer concentrations, and confirmed theoretical expectations that the area of oligomer peaks was proportional to the molar concentration of the oligomer species. The effect of pressure on hydrolysis was tested by superpressurizing reactors with nitrogen - no pressure effect on hydrolysis was detected.

Experiments were conducted to confirm $\mathrm{pH}$ predictions of $\mathrm{CO}_{2}$ by comparing results to dilute $\mathrm{H}_{2} \mathrm{SO}_{4}$. Hydrolysis was done by subjecting a $1 \mathrm{~g} / \mathrm{L}$ xylan solution to $\mathrm{H}_{2} \mathrm{SO}_{4}$ and/or $\mathrm{CO}_{2}$ or $\mathrm{N}_{2}$ at $190^{\circ} \mathrm{C}$ or $200^{\circ} \mathrm{C}$ for 16 minutes.
\end{abstract}

\section{Materials and Methods}

Apparatus and Materials: DX500 Chromatography System (consists of Dionex GP50 gradient pump, ED40 electrochemical detector, AS3500 autosampler), Xylan (sigma), xylose (Sigma), 15 mL 316 stainless steel reactor vessels, glass serum vials, Techne SBL-2D fluidized aluminum oxide sand baths, Pharmco sulfuric acid at 95.0 to $98.0 \%, 18 \mathrm{ohm}$ high resistance water, deionized water, spigot-attached vacuum apparatus with tubing, ultra pure helium gas, Eppendorf micro pipettes (various sizes), DU Series 500 Beckman Spectrophotometer, Eppendorf centrifuge 5417C, and centrifuge tubes. Fisher Scientific AR15 pH meter by Accumet Research; Ohaus Explorer digital scale Item \#12140 (d=0.1mg); Pyrex glass beakers, graduated cylinders, burettes and flasks;

\section{Procedures:}

Solution preparation:_A 1-g/L xylan solution was prepared with 0.25 -g of dried xylan into $250 \mathrm{~mL}$ of DI water. For hydrolysis in $1 \% \mathrm{H}_{2} \mathrm{SO}_{4}, 18 \mathrm{M}$ acid was added to the xylan solution to give a $1 \%$ solution. The acidified xylan was placed on an active stir plate with a magnetic stir bar in solution. For hydrolysis with varying $\mathrm{pH}$ conditions, $18 \mathrm{M} \mathrm{H}_{2} \mathrm{SO}_{4}$ was added to a 1 $\mathrm{g} / \mathrm{L}$ xylan solution in varying amounts. The xylan solution was placed on an active stir plate with a magnetic stir bar in solution. It was then pipetted into the steel reactor vessels in 10$\mathrm{mL}$ aliquots. The acid solution, in a range of $\mathrm{pHs}$, would next be added to the reactor vessel in $1.1 \mathrm{~mL}$ aliquots.

Hydrolysis: For experiments at 121C, the acidified xylan solution was pipetted into the glass reactor vessels in $10-\mathrm{mL}$ aliquots. The vial was sealed with a rubber stopper and an aluminum crimping ring. Each vial was placed in the sand bath for the desired time. For reactions at higher temperature, 316 stainless steel reactors were used, pressurized with $\mathrm{CO}_{2}$ if necessary, and placed in a sand bath $\left(190^{\circ} \mathrm{C}\right.$ or $\left.200^{\circ} \mathrm{C}\right)$ for 16 minutes. The samples were frozen for a later time to be analyzed by HPAE. Samples were run in duplicate. 
Analysis: The samples were analyzed for polymer distribution using the HPAE. .The HPAE consists of a pump, a separation column, and a detector. Each prepared sample was pumped through the column to distinguish the amount of hydrolysis occurring at each time interval. To prepare for the run, a $500 \mathrm{mmol} \mathrm{NaOH}$ solution was made and degassed for use as an eluent. Each of the samples was diluted by 1 in 20 with deionized water. A set of standards was prepared using a $1 \mathrm{~g} / \mathrm{L}$ xylose solution. The stock xylose solution was diluted by 1:20, 1:25, 1:33, 1:50 and 1:100 to obtain standards at $50 \mathrm{mg} / \mathrm{L}, 40 \mathrm{mg} / \mathrm{L}, 30 \mathrm{mg} / \mathrm{L}, 20 \mathrm{mg} / \mathrm{L}$ and 10 $\mathrm{mg} / \mathrm{L}$, respectively. The acidified xylose samples were further analyzed using a DU Series 500 Spectrophotometer to observe absorbency in the ultra-violet light range.

pH prediction: Most of the reactions carried out for testing the $\mathrm{pH}$ of carbonic acid at elevated temperature were done at $190 \mathrm{C}$ for 16 minutes. This gives a $\log$ (severity) value of 3.85, where the severity function in its log form is calculated as per equation (1), first defined by Overend and Chornet (1987):

$$
\log \left(R_{O}\right)=\log _{10}(t \times \exp ((T-100) / 14.75))
$$

Where $R_{O}$ is the severity, $t$ is the reaction time expressed in minutes and $T$ is the temperature expressed in degrees Celcius. The combined severity values for the experimental conditions, with the $\mathrm{CO} 2$ at an initial, room temperature pressure of 800 psi is 0.27 . The combined severity function was defined by Chum et al. (1990) as:

$$
C S=\log \left(R_{O}\right)-\mathrm{pH}
$$

Where CS signifies the combined severity. In applying the combined severity factor to the carbonic acid system, van Walsum (2001) suggested the following equation, which estimates the value of the solution $\mathrm{pH}$ from the temperature and the partial pressure of the $\mathrm{CO}_{2}$ :

$$
C S_{P_{\mathrm{CO} 2}}=\log \left(R_{O}\right)-8.00 \times T^{2}+0.00209 \times T-0.216 \times \ln \left(\mathrm{PCO}_{2}\right)+3.92
$$

Where $C S_{\mathrm{Pco}_{2}}$ is the combined severity determined from the partial pressure of $\mathrm{CO}_{2}$ and $\mathrm{PCO}_{2}$ is the partial pressure of $\mathrm{CO}_{2}$ in atmospheres.

The use of the term "partial pressure" under these conditions is somewhat misleading, since the temperature and pressure range of the pretreatment put $\mathrm{CO}_{2}$ into its supercritical phase, while the water vapor is still present in the gas phase. Thus, equation (3) pertains more precisely to the fugacity of the $\mathrm{CO}_{2}$ in the non-liquid phase. For a component in solution, fugacity and partial pressure are related by

$$
\varnothing_{\mathrm{i}}=f \mathrm{i} / \mathrm{y}_{\mathrm{i}} \mathrm{P}
$$

Where $\varnothing_{\mathrm{i}}$ is the fugacity coefficient of component $\mathrm{i}, f_{\mathrm{i}}$ is the fugacity, $\mathrm{y}_{\mathrm{i}}$ is the molar fraction and $\mathrm{P}$ the overall pressure of the system (Smith and Van Ness, 1975). For a binary i,j gas mixture, the multicomponent Virial equation simplifies to:

$$
\ln \varnothing_{\mathrm{i}}=\frac{P}{\mathrm{RT}}\left(B_{\mathrm{ii}}+\mathrm{y}_{\mathrm{j}}^{2} \delta_{\mathrm{ij}}\right) \quad \text { with } \delta_{\mathrm{ij}}=2 B_{\mathrm{ij}}-B_{\mathrm{ii}}-B_{\mathrm{jj}}
$$

Where $\mathrm{R}$ is the universal gas constant, $T$ is the temperature in degrees Kelvin, $\mathrm{y}_{\mathrm{j}}$ is the molar fraction of the solvent in the gas phase and $B_{\mathrm{ij}}$ is the second virial coefficient, which can be expressed by equation (6) (Smith and Van Ness, 1975) 


$$
B_{\mathrm{ij}}=\frac{\mathrm{R} T_{\mathrm{Cij}}}{P_{\mathrm{Cij}}}\left(B^{0}+\omega_{\mathrm{ij}} B^{1}\right)
$$

In which $\omega$ is the acentric factor. The parameters $\omega_{\mathrm{ij}}, \mathrm{T}_{\mathrm{cij}}$, and, $\mathrm{P}_{\mathrm{cij}}$ were calculated with mixing rules presented in Smith and Van Ness (1975), as follows:

$$
\begin{aligned}
& \omega_{\mathrm{ij}}=\left(\omega_{\mathrm{i}+} \omega_{\mathrm{j}}\right) / 2 \\
& T_{\mathrm{cij}}=\left(T_{\mathrm{ci}} T_{\mathrm{cj}}\right)^{1 / 2} \\
& P_{\mathrm{cij}}=\left(Z_{\mathrm{cij}} \mathrm{R} T_{\mathrm{cij}}\right) / V_{\mathrm{cij}}
\end{aligned}
$$

In which $Z$ is the compressibility. $Z_{\mathrm{cij}}$ and $V_{\mathrm{cij}}$ are determined by:

$$
\begin{aligned}
& Z_{\mathrm{cij}}=\left(Z_{\mathrm{ci}}+Z_{\mathrm{cj}}\right) / 2 \\
& \mathrm{~V}_{\mathrm{cij}}=\left(\left(\mathrm{V}_{\mathrm{ci}}^{1 / 3}+\mathrm{V}_{\mathrm{cj}}^{1 / 3}\right) / 2\right)^{3}
\end{aligned}
$$

$B^{0}$ and $B^{1}$ were calculated as proposed in Smith and Van Ness (1975), as follows:

$$
\begin{aligned}
& B^{0}=0.083-0.422 / \mathrm{T}_{\mathrm{r}}^{1.6} \\
& B^{1}=0.139-0.172 / \mathrm{T}_{\mathrm{r}}^{4.2}
\end{aligned}
$$

These equations can be solved iteratively starting with the known temperature, total pressure and assumed values for molar fractions. Initial estimates for molar fractions were obtained by assuming ideal gas behavior, which yielded convergent results. Results from these calculations for several reaction conditions are summarized in table 1a.1. 
Table 1a.1. Results of thermodynamic determination of carbonic acid $\mathrm{pH}$ at elevated temperatures and pressures.

\begin{tabular}{|c|c|c|c|c|c|c|}
\hline $\begin{array}{c}\text { Temperature } \\
\text { C }\end{array}$ & $\begin{array}{c}\text { Total } \\
\text { pressure } \\
\text { (psia) }\end{array}$ & $\mathrm{yco}_{2}$ & Z & $\varnothing$ & $\begin{array}{c}\mathrm{fCO}_{2} \\
\text { (psia) }\end{array}$ & $\mathrm{pH}$ \\
\hline 180 & 1941 & .925 & .850 & .861 & 1606 & 3.54 \\
\hline 190 & & & & & 1686 & 3.58 \\
\hline 200 & 2165 & .896 & .854 & .864 & 1766 & 3.62 \\
\hline 210 & & & & & 1845 & 3.67 \\
\hline 220 & 2416 & .86 & .872 & .880 & 1924 & 3.71 \\
\hline
\end{tabular}

Previously reported [van Walsum, 2001] calculations for the $\mathrm{pH}$ of high temperature carbonic acid, which did not incorporate the fugacity coefficient calculations shown above, had predicted a $\mathrm{pH}$ of 3.56 at $190 \mathrm{C}$. This result is in very near agreement with the more thermodynamically correct value calculated in table 1a.1, and indicates that simplified assumptions about the gas phase behavior of $\mathrm{CO} 2$ may still be viable under these conditions.

\section{Results}

Oligomer quantification

Figure 1a.1 shows results from xylan hydrolysis at $121 \mathrm{C}$ in $1 \%$ sulfuric acid. It can be seen that at about time 10 minutes, all of the $1 \mathrm{~g} / \mathrm{L}$ xylan originally present has been hydrolyzed to oligomers small enough to be quantified by the HPAE. The relative stability of total oligomer concentration at times above 10 minutes demonstrates that the quantification of oligomers is relatively robust, since for all these cases the mass balance comes close to closure. At times less than 10 minutes, insufficient hydrolysis had occurred and large DP oligomers were not being detected. 
Figure 1a.1 Total xylose and xylan oligomers released in $1 \% \mathrm{H}_{2} \mathrm{SO}_{4}$ hydrolysis of $1 \mathrm{~g} / \mathrm{L}$ xylan at $121 \mathrm{C}$.

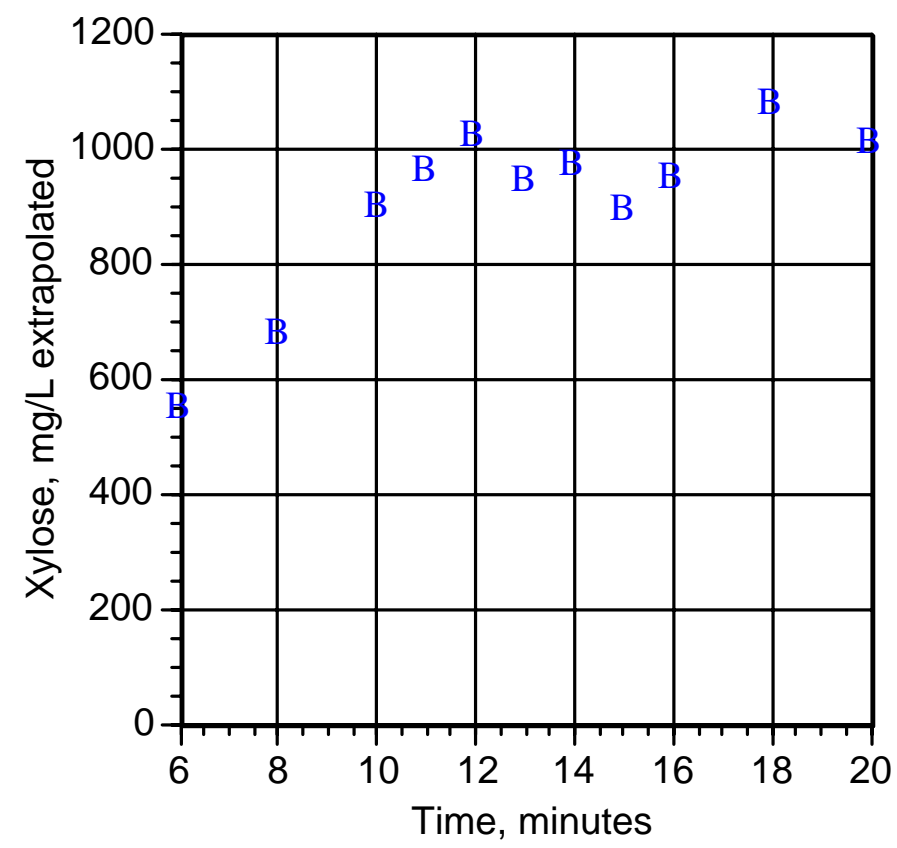

B wtd areas as xylose

pH confirmation

Figure 1a. 2 shows results of dilute sulfuric acid and carbonic acid hydrolysis of $1 \mathrm{~g} / \mathrm{L}$ xylan at $190 \mathrm{C}$. The figure shows the effect of combining carbonic and sulfuric acids for hydrolysis of xylan. In this experiment it can be seen that down to a $\mathrm{pH}$ of about 3.2, carbonic acid is able to supplement the hydrolysis of the sulfuric acid. Below pH 3.2 the carbonic acid can no longer contribute to enhancing hydrolysis. It appears that carbonic acid on its own has an effective $\mathrm{pH}$ of about 3.4 Compared to the theoretical value of 3.58, this appears to show minor deviation from theoretically expected results. Van Walsum[2001] found similar deviation results, where the observed hydrolysis was equivalent to sulfuric acid at 3.37 and the predicted $\mathrm{pH}$, using idealized gas calculations, had been 3.56 . 
Figure 1a.2: Xylose accumulation in varying $\mathrm{pH}$ of $\mathrm{H}_{2} \mathrm{SO}_{4}$ and $\mathrm{CO}_{2}$ with varying $\mathrm{pH}$ of $\mathrm{H}_{2} \mathrm{SO}_{4}$

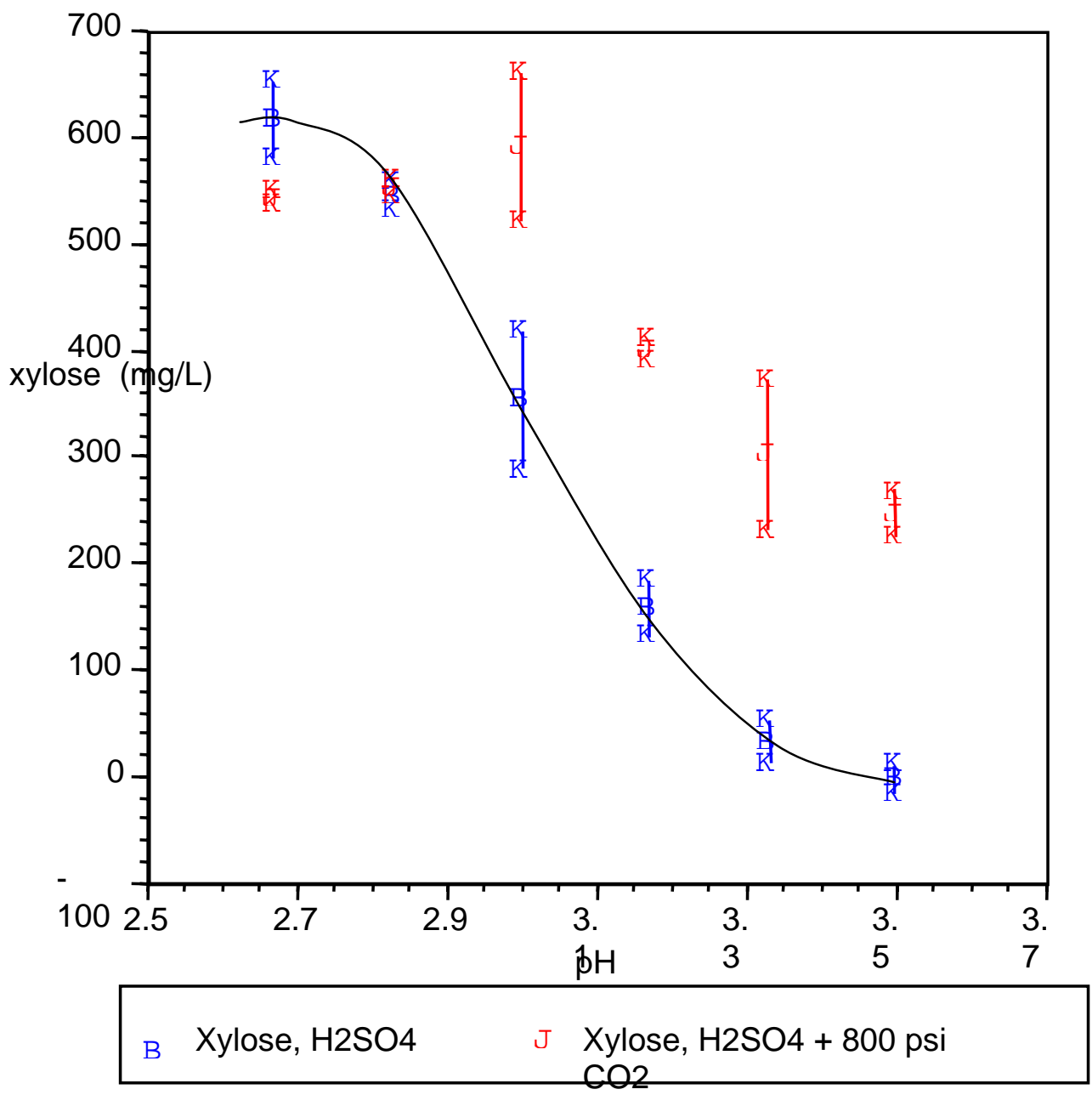

\section{Discussion \& Conclusions}

It was found that oligomer quantification based on a correlation of peak area to molar concentration of oligomers served to close a mass balance on partially hydrolyzed xylan solutions. This enabled quantification of xylan solubilization in cases of incomplete hydrolysis.

In the varying $\mathrm{pH}$ experiments, $\mathrm{CO}_{2}$ at 800 psig most closely approximated $\mathrm{H}_{2} \mathrm{SO}_{4}$ at a $\mathrm{pH}$ of 3.4 and appeared to be able to assert some hydrolytic activity in the presence of other acids down to a $\mathrm{pH}$ of 3.2. Predictions of the $\mathrm{pH}$ of carbonic acid appear to have over estimated the effective $\mathrm{pH}$ by approximately $0.18 \mathrm{pH}$ units. These results are in agreement with previously published findings.

\section{References}


Van Walsum, G. Peter, 2001. Severity function Describing the Hydrolysis of Xylan Using Carbonic Acid.

Smith, J.M, and van Ness, H.C. 1975. Introduction to Chemical Engineering Thermodynamics, 3rd ed'n. McGraw Hill Book Company. 


\title{
TASK 1b) Arrhenius equation for $\mathrm{H}_{2} \mathrm{CO}_{3}$
}

\author{
Vanessa Castleberry, G. Peter van Walsum
}

\section{Summary:}

Experiments were conducted to develop an Arrhenius rate constant for sulfuric acid and $\mathrm{CO}_{2}$. These experiments consisted of hydrolyzing xylan at varying temperatures and initial pressures of $\mathrm{CO}_{2}$, while maintaining a constant $\mathrm{pH}$ of 3.4. Comparative results were generated using $\mathrm{H}_{2} \mathrm{SO}_{4}$. To characterize the species in the hydrolysate, samples were analyzed by $\mathrm{pH}$, HPAE and UV absorbency in a spectrophotometer.

\section{Materials and Methods:}

Apparatus and Materials: DX500 Chromatography System (consists of Dionex GP50 gradient pump, ED40 electrochemical detector, AS3500 autosampler), Xylan (sigma), xylose (Sigma), $15 \mathrm{~mL} 316$ stainless steel reactor vessels, glass serum vials, Techne SBL-2D fluidized aluminum oxide sand baths, Pharmco sulfuric acid at 95.0 to $98.0 \%, 18 \mathrm{ohm}$ high resistance water, deionized water, spigot-attached vacuum apparatus with tubing, ultra pure helium gas, Eppendorf micro pipettes (various sizes), DU Series 500 Beckman Spectrophotometer, Eppendorf centrifuge 5417C, and centrifuge tubes. Fisher Scientific AR15 pH meter by Accumet Research; Ohaus Explorer digital scale Item \#12140 (d=0.1mg); Pyrex glass beakers, graduated cylinders, burettes and flasks;

\section{Procedures:}

Solution preparation: A new 1-g/L xylan solution was prepared with $2 \mathrm{~g}$ of dried xylan into 2 $\mathrm{L}$ of DI water. For hydrolysis in $1 \% \mathrm{H}_{2} \mathrm{SO}_{4}, 18 \mathrm{M}$ acid was added to the xylan solution in varying amounts to observe responses to a range of $\mathrm{pHs}$ side by side with $\mathrm{CO}_{2}$. The xylan was placed on an active stir plate with a magnetic stir bar in solution.

Hydrolysis: The solution was pipetted into $150 \mathrm{~mL}, 316$ stainless steel reactor vessels in 10$\mathrm{mL}$ aliquots. To maintain a constant $\mathrm{pH}$ for the acids over the temperature range, the temperature dependence of the acid dissociations was taken into account when preparing solutions for use at different temperatures. This resulted in the lower temperature solutions being reacted at lower pressures of $\mathrm{CO} 2$ and lower concentrations of sulfuric acid. The reactor was closed, and if dictated, pressurized, and placed in a sand bath $\left(170^{\circ} \mathrm{C}\left(210^{\circ} \mathrm{C}\right.\right.$ preheat $)$, $180^{\circ} \mathrm{C}\left(220^{\circ} \mathrm{C}\right.$ preheat $)$, or $190^{\circ} \mathrm{C}\left(230^{\circ} \mathrm{C}\right.$ preheat $)$ for 14.5 minutes. Samples were preheated for 3 minutes in the $150 \mathrm{~mL}$ reactor. The samples were refrigerated until HPAE analysis was run. Samples were run in duplicate.

Analysis: The samples were analyzed using the HPAE. To prepare for the run, a 500mmol $\mathrm{NaOH}$ solution was made and degassed for use as an eluent. Each of the samples was diluted by 1 in 20 with deionized water. A set of standards was prepared using a 100mg/L-xylose solution. The xylose solution was diluted by $1: 2,1: 2.5,1: 3.3$, 1:5 and $1: 10$ to obtain standards at $50 \mathrm{mg} / \mathrm{L}, 40 \mathrm{mg} / \mathrm{L}, 30 \mathrm{mg} / \mathrm{L}, 20 \mathrm{mg} / \mathrm{L}$ and $10 \mathrm{mg} / \mathrm{L}$. Each prepared sample was pumped through the column to distinguish the amount of hydrolysis occurring at each time interval.

The acidified xylose samples were further analyzed using a DU Series 500 Spectrophotometer to observe absorbency at $275 \mathrm{~nm}$. 


\section{Results:}

Results from the hydrolysis experiments are presented in figures $1 \mathrm{~b} .1$ and 1b.2, which show results for the carbonic and sulfuric acid systems, respectively. The slopes of the plots indicate activation energies of $11.9 \mathrm{~kJ} / \mathrm{mol}$ for the sulfuric acid system and $12.6 \mathrm{~kJ} / \mathrm{mol}$ for the carbonic acid system. It is not clear whether this difference is significant in terms of identifying a difference in the effective hydrolysis activity of the two acids. The $\mathrm{R}^{2}$ values for the slopes of these plots are .988 and .976 respectively, which demonstrates a relatively good fit to the data in each case, but does not instill confidence in the significance of the effect.

\section{Figure 1b.1 Plot of 1/T versus $\ln (\mathrm{K})$ for carbonic acid hydrolysis of xylan}

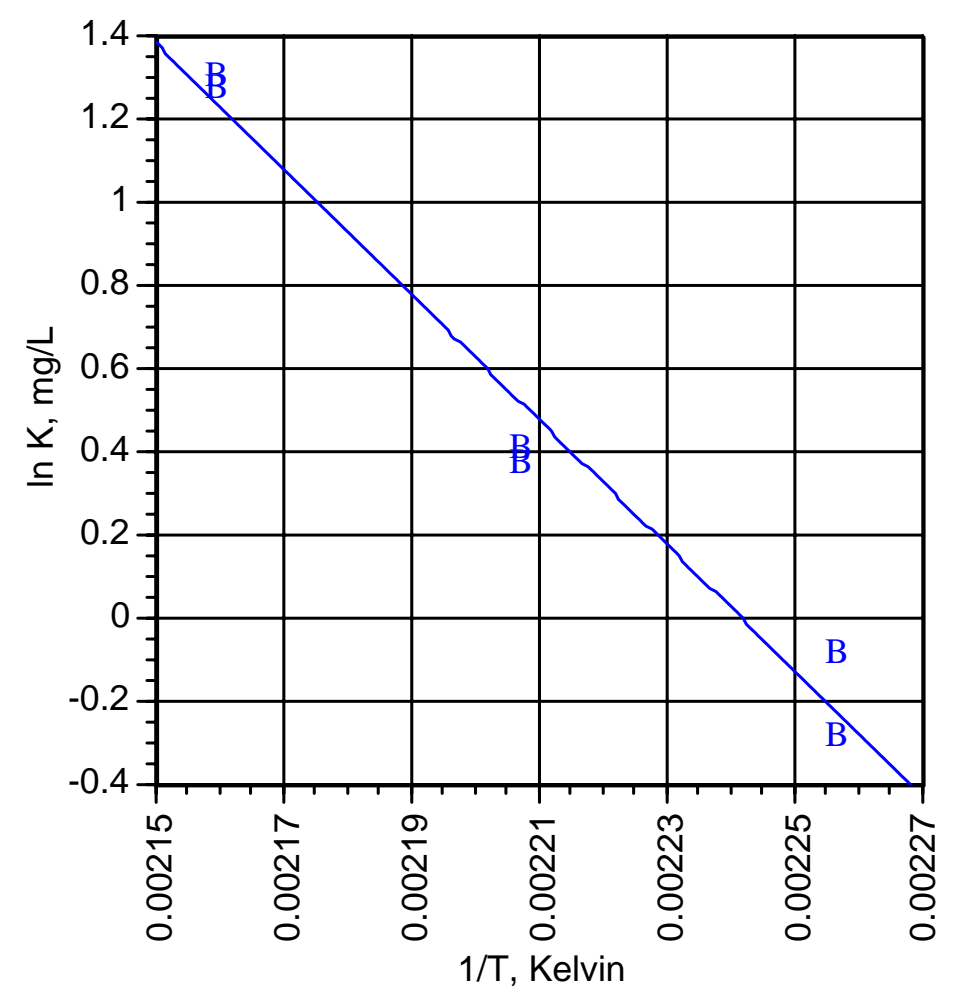

B In K psi, corrected 
Figure 1b.2 Plot of 1/T versus $\ln (\mathrm{K})$ for sulfuric acid hydrolysis of xylan

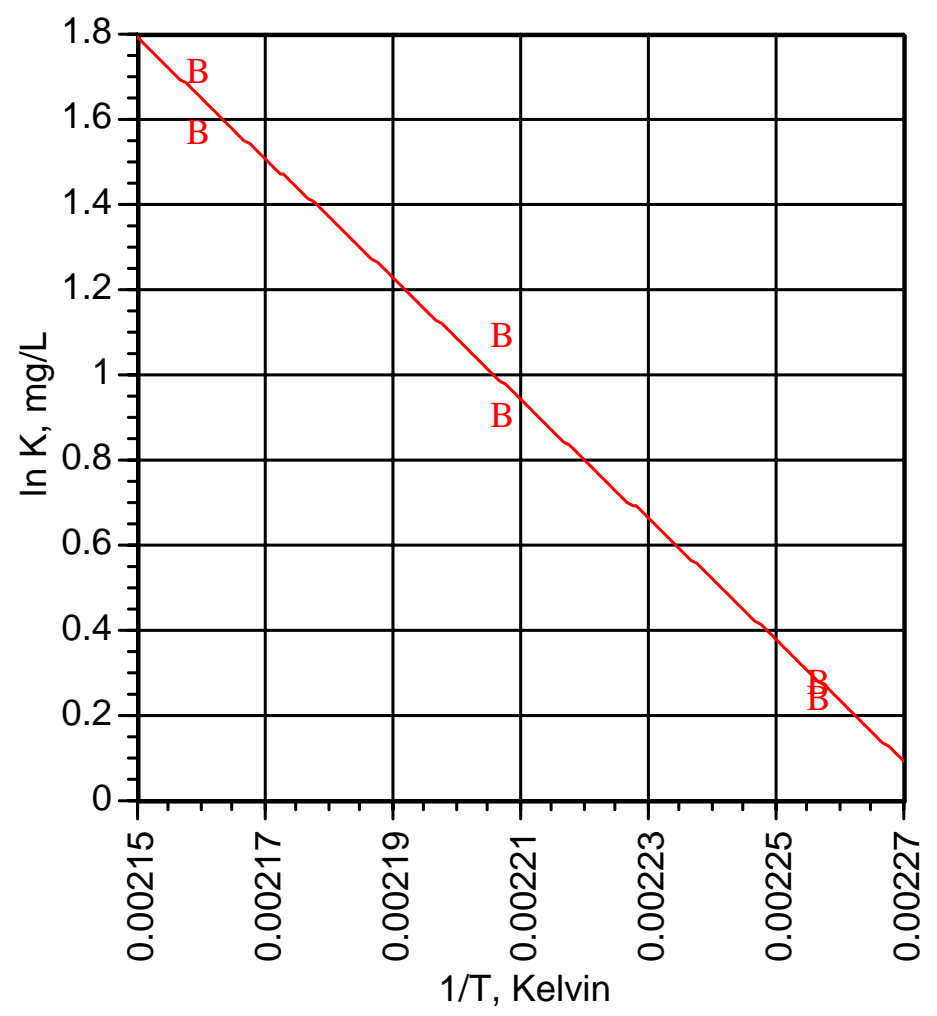

B $\ln \mathrm{K} \mathrm{pH}$, corrected

\section{Discussion and Conclusions}

It appeared relatively straight forward to calculate the activation energy for both sulfuric and carbonic acids. There does not appear to be any significant effect on hydrolysis that could be attributed to the type of acid used. Temperature effects on the acid dissociations were taken into account when preparing the samples to achieve a constant $\mathrm{pH}$ at each temperature. 


\title{
TASK 1c) Buffering Capacity of $\mathrm{H}_{2} \mathrm{CO}_{3}$
}

\author{
Vanessa Castleberry, G. Peter van Walsum
}

\begin{abstract}
Summary:
Repeat experiments of aspen samples were hydrolyzed and titrated against a standardized $\mathrm{NaOH}$ solution to determine molar concentration of acid species in the hydrolysate. In some experiments it appeared that the acid concentration of hydrolysate of reactions with $\mathrm{CO}_{2}$ was significantly lower than the hydrolysate of reactions of wood and water alone. This confirms earlier work that consistently measured a higher $\mathrm{pH}$ in carbonic acid pretreated hydrolysates, compared to water-only pretreatment. However, in other titration experiments, the opposite result was observed, with the carbonic acid system having more accumulated acids present.
\end{abstract}

\section{Introduction}

During previous experimentation using aspen wood, the hydrolysate $\mathrm{pH}$ of reactions with $\mathrm{CO}_{2}$ was higher than reactions without. Since acetic acid may likely be the major contributor of $\mathrm{H}+$ ions into solution, it is hypothesized that the presence of $\mathrm{CO}_{2} /$ carbonic acid may somehow reduce the release of acetyl groups, or in some other way reduce the activity of acid species in solution. This experiment attempted to close the "acid balance" by titrating hydrolysates and comparing titration results to analytical determination of acetic and formic acids in solution.

\section{Materials + Methods}

Apparatus and Materials: DX500 Chromatography System (consists of Dionex GP50 gradient pump, ED40 electrochemical detector, AS3500 autosampler), xylose (Sigma), 15 mL 316 stainless steel reactor vessels, Techne SBL-2D fluidized aluminum oxide sand baths, J. T. Baker Glacial Acetic acid, Pharmco sulfuric acid at 95.0 to $98.0 \%$, Mallinckrodt $\mathrm{AR}^{\circledR}$ sodium hydroxide solution at 50\% w/w, Tetrabutylammonium hydroxide solution, $40 \% \mathrm{w} / \mathrm{w}$; Heptafluorobutyric acid solution, 99\%; 18 ohm high resistance water, deionized water, Fisher heated stirring plate, magnetic stir bars, glass beakers, Erlenmeyer flasks, Kjeldahl flasks, spigot-attached vacuum apparatus with tubing, ultra pure helium gas, Eppendorf micro pipettes (various sizes), DU Series 500 Beckman Spectrophotometer, Eppendorf centrifuge 5417C, and centrifuge tubes. Fisher Scientific AR15 pH meter by Accumet Research; Swagelock 150ml stainless steel reactor; Ohaus Explorer digital scale Item \#12140 (d=0.1mg); Pyrex glass beakers, graduated cylinders, burettes and flasks; Aspen wood; Aspen hydrolysates; Aspen solids.

\section{Procedures:}

Hydrolysis: A sand bath was heated to $190{ }^{\circ} \mathrm{C}$, another $230{ }^{\circ} \mathrm{C}$. Aspen was ground and sifted, using the particles of $1 \mathrm{~mm} .1 .0 \mathrm{~g}$ of aspen was weighed and placed in 150-mL stainless steel reactor, along with $80 \mathrm{~mL}$ of water. Using 3 reactors, simultaneously, all samples at each parameter were generated at once. Depending on the sample, the reactor was charged either with water and wood or with water, wood and $800 \mathrm{psig}$ of $\mathrm{CO}_{2}$. The filled reactors were placed in the $230^{\circ} \mathrm{C}$ sand bath for a 3-minute preheat. Upon completion of preheat time, the reactors were transferred to $190^{\circ} \mathrm{C}$ sand bath for 15 minutes of reaction time. Reactions were quenched in ice bath after 15 minutes at target temperature.

Titrations: 2 L of ultra pure water were brought to boil for 20-30 minutes. Remove from heat. The water was stoppered to cool and transferred to polyethylene bottles that were tightly 
closed. A titrant of $\sim 0.01 \mathrm{M} \mathrm{NaOH}$ was mixed up, using newly bottled water. The titrant was stored in a tightly closed polyethylene container. KHP (Potassium Hydrogen Phthalate) was weighed and dried in a $100^{\circ} \mathrm{C}$ oven overnight. The container was transferred to a dessicator and allowed it to cool. The \# of moles in $20 \mathrm{~mL}$ of the newly made $\mathrm{NaOH}$ solution was calculated and, in the same number of moles of KHP the weight was calculated. That amount was weighed into a $125 \mathrm{~mL}$ flask. Enough degassed water was added to dissolve it ( $20 \mathrm{~mL})$. Fresh $\mathrm{NaOH}$ was poured into a $25 \mathrm{~mL}$ burette. The $\mathrm{pH}$ probe was placed into the KHP solution and titrated against the $\mathrm{NaOH}$ solution. The $\mathrm{mV}$ (potential) and $\mathrm{pH}$ were recorded after each $\mathrm{mL}$ of $\mathrm{NaOH}$ was added. Using the derivative of the end point, the exact concentration of $\mathrm{NaOH}$ solution was calculated. The exact molarity of the $\mathrm{NaOH}$ solution was $9.91954 * 10^{-3}$. Each hydrolysate titration was repeated 4 times and the average was used to derive an end point. For each tirtration, $25 \mathrm{ml}$ of hydrolysate was placed in a $150 \mathrm{ml}$ beaker which was then placed on a stir plate. The $\mathrm{pH}$ probe was placed in the beaker as well. The $0.1 \mathrm{M} \mathrm{NaOH}$ was added incrementally (between $0.1 \mathrm{ml}$ and 1.0 ) $\mathrm{ml}$ and the $\mathrm{pH}$ was measured and recorded at each increment. The data will be plotted and evaluated to determine the number and concentrations of acids present in the hydrolysate.

\section{Results:}

Figures 1c.1 to 1c.7 show results from different experiments doing titrations on aspen wood hydrolysates. Figures 1c.1, 1c.4, 1c.6 show results of water pretreated aspen wood, figures 1c.2, 1c.5, 1c.7 carbonic acid pretreated aspen wood. Figure 1c.3 shows the calibration titration using KHP. It can be seen that in the experiment that generated figures 1c.1 and 1c.2, the wood hydrolysate has less overall acid than the carbonic acid hydrolysate, indicated by the lower volume of $\mathrm{NaOH}$ solution needed to reach the inflection point in the titration curve. Figures 1c.4 and 1c.5 show little difference between the two titrations, while 1c.6 and 1c.7 show both qualitative and quantitative differences: the $\mathrm{CO}_{2}$ system has a much more gradually buffered-looking titration curve, indicating a variety of acid species. 
Figure 1c.1 Titration of aspen hydrolysate without $\mathrm{CO}_{2}$

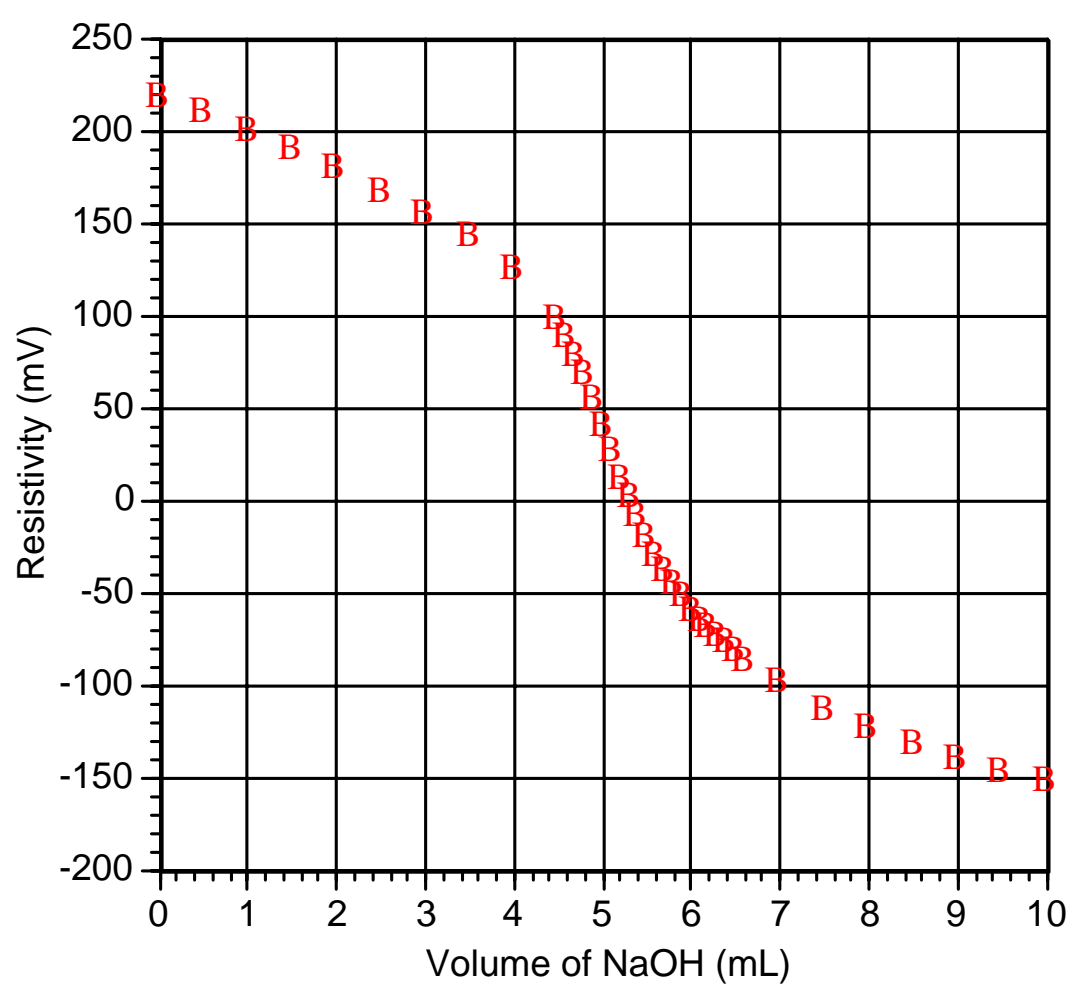

B Avg no $\mathrm{CO} 2$

Figure 1c.2 Titration of aspen hydrolysate with $\mathrm{CO}_{2}$

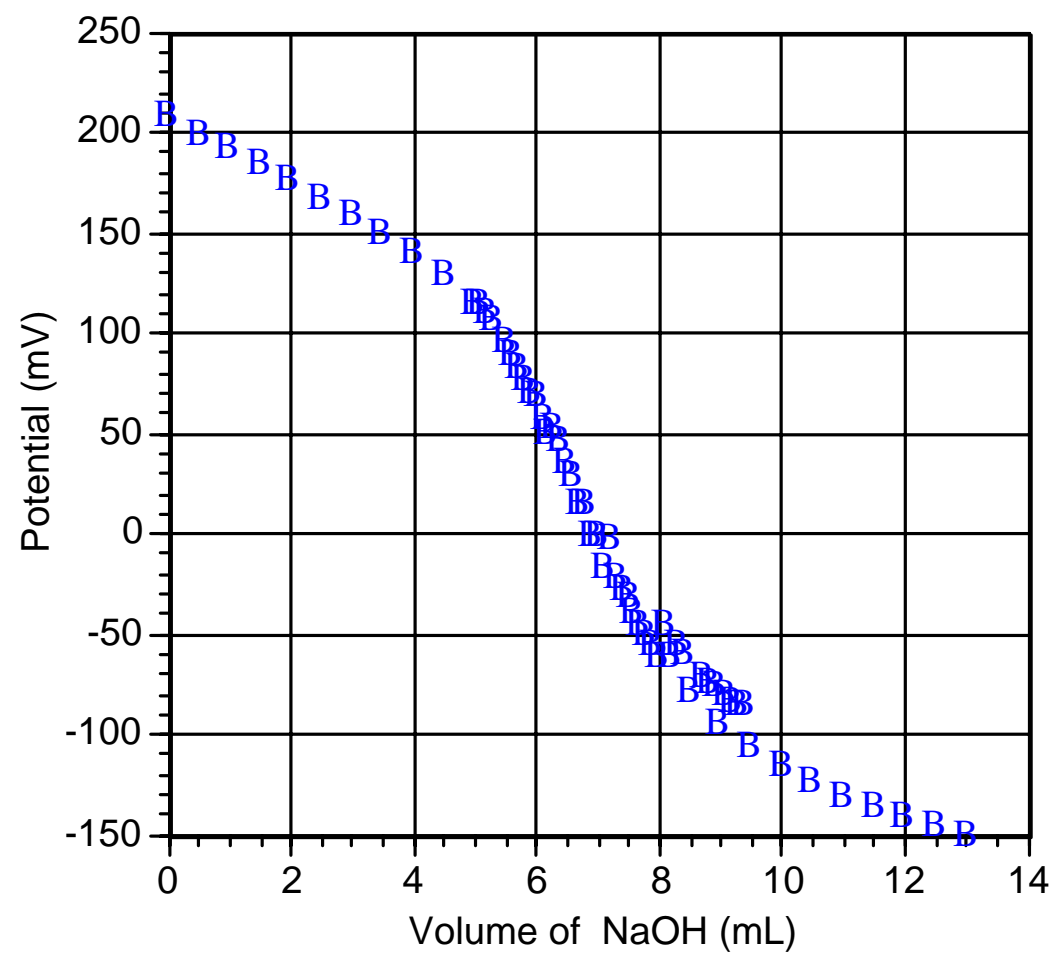

B Average $\mathrm{mV}$ 
Figure 1c.3: KHP Standard Titration curve

KHP Titration

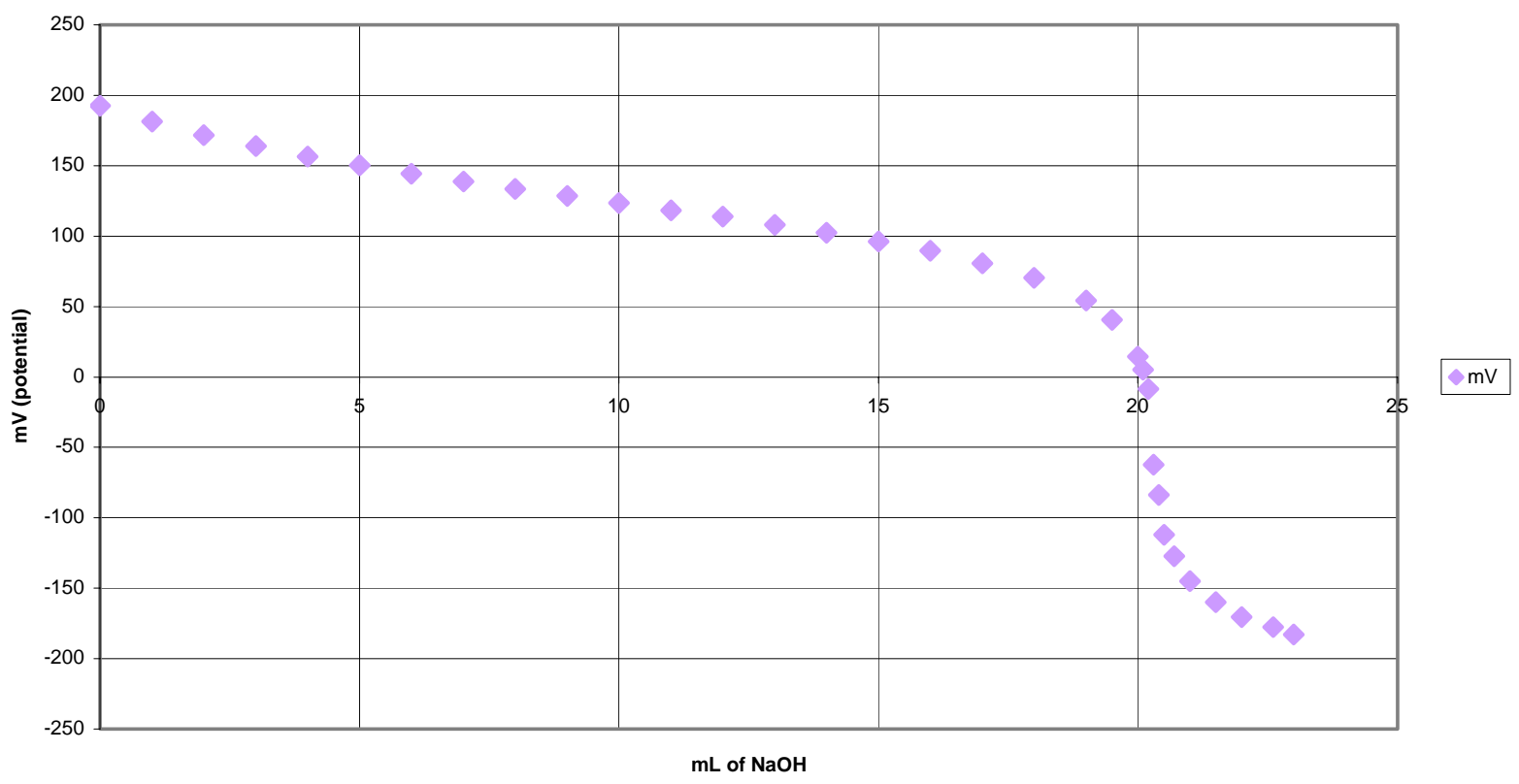


Figure 1c.4: Titration Curve of Water Pretreated Aspen

Average Titration of Aspen without $\mathrm{CO} 2$

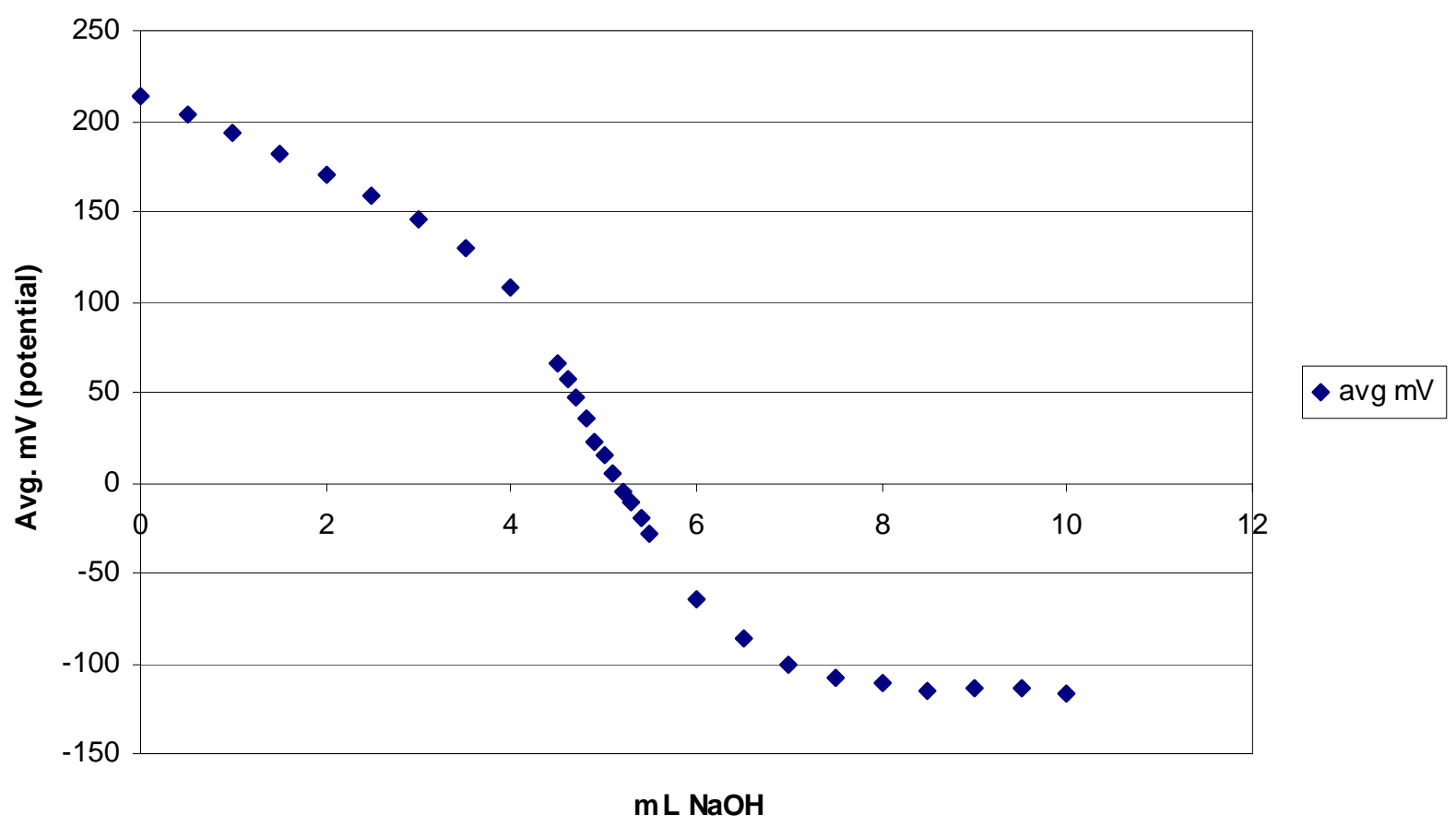

Figure 1c.5 Titration of Carbonic Acid Pretreated Aspenwood

Titration Average of Hydrolysate with $\mathrm{CO} 2$

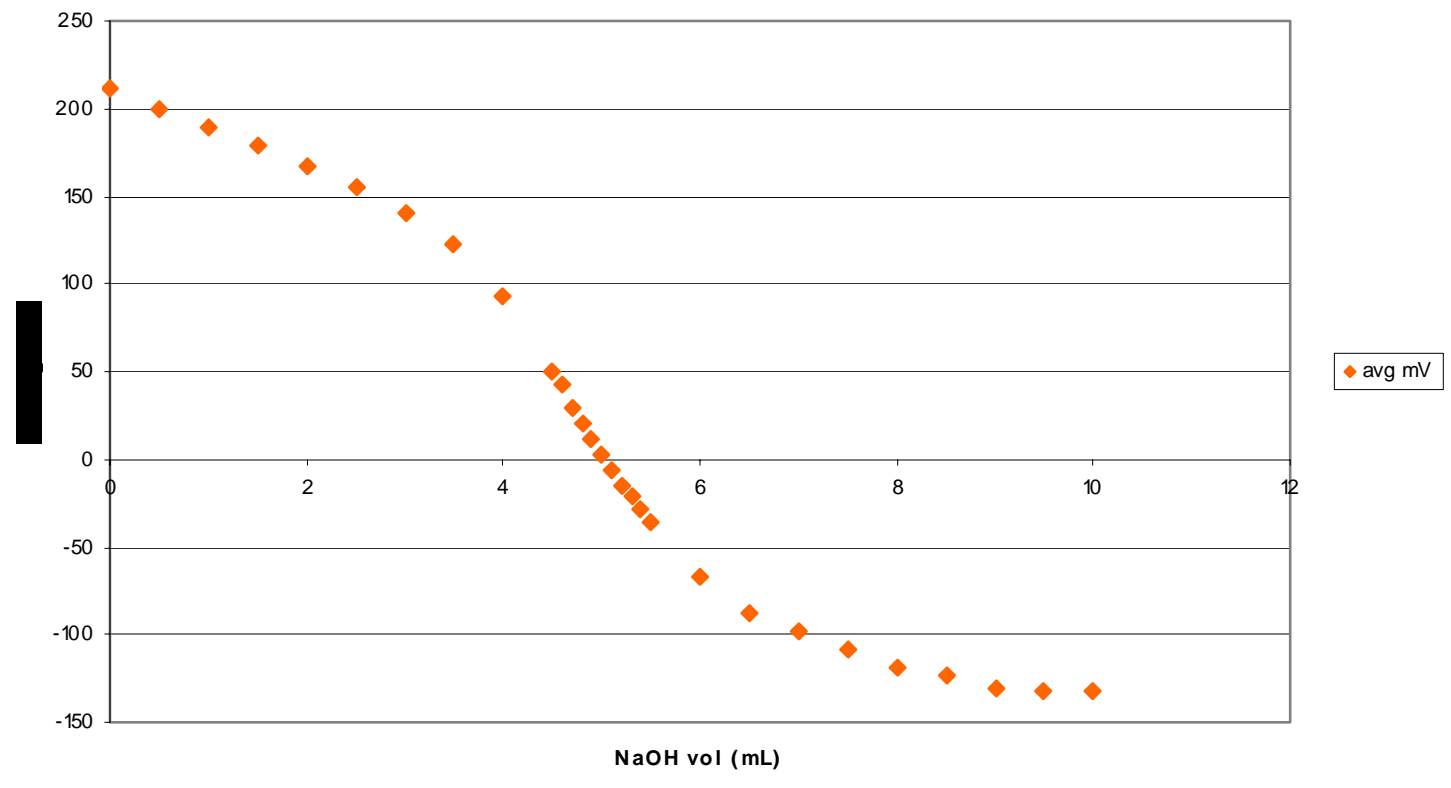


Figure 2c.6

pH Titration Results for Neutralization of Hydrolysate from Liquid Hot Water Pretreatment

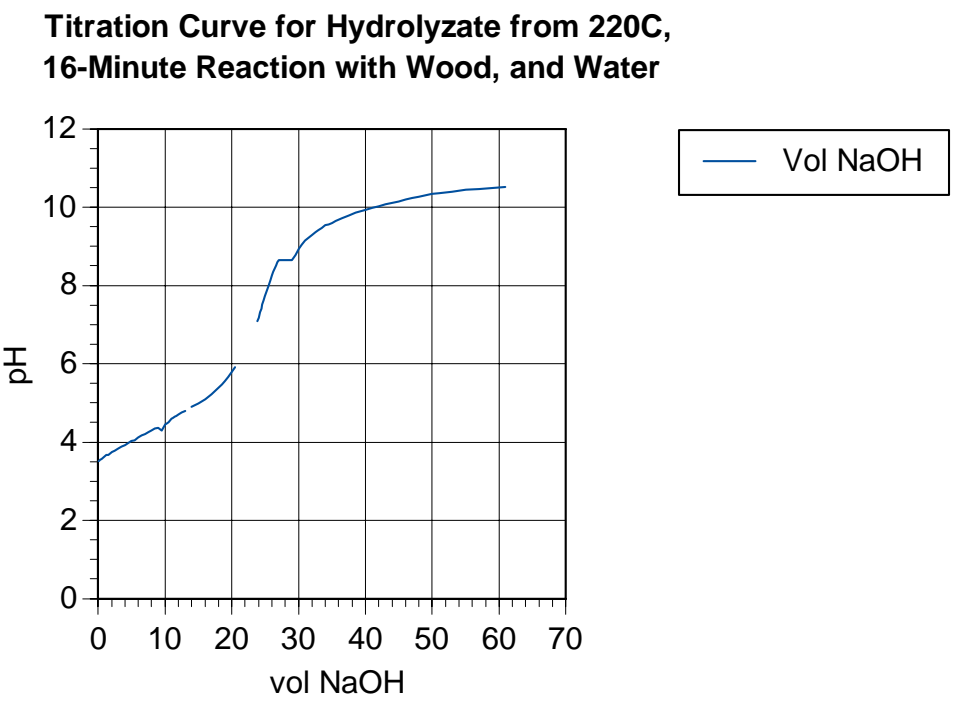

Figure 2c.7

pH Titration Results for Neutralization of Hydrolysate from Carbonic Acid Pretreatment

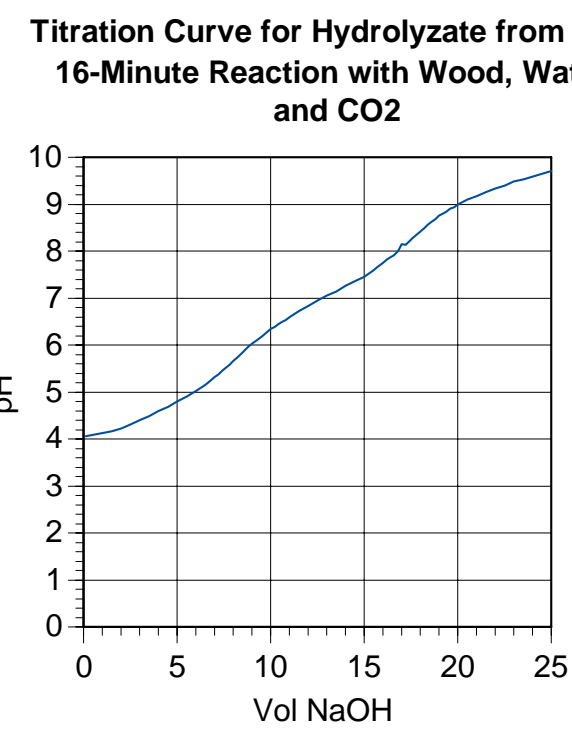




\section{Discussion and Conclusions}

The curious, and previously reported [1] result of carbonic acid having a net effect of increasing the final aspen wood hydrolysate $\mathrm{pH}$ has been confirmed for most experiments, but this result is not consistent with allof the titration studies undertaken. At this point there are inconsistent results in the titration results and so no conclusions can be drawn. Further work is being done to increase the reproducibility of these results. 
TASK 2 Perform laboratory experiments on the pretreatment of raw biomass

\author{
John Lam, Robert McWilliams, Connaly Miller, G. Peter van Walsum
}

\begin{abstract}
Summary
A data set of pretreatment results on aspen using carbonic acid had been completed prior to the start of this project [1]. Experiments continued to increase the reliability of the results and to achieve uniform performance between laboratory personnel. These objectives were achieved and reduced the scatter and uncertainty in the results.
\end{abstract}

Experiments were also carried out to determine whether the rate of mass transfer of $\mathrm{CO}_{2}$ into or out of solution exerts an effect on the rate of hydrolysis. It was found that extra $\mathrm{CO}_{2}$ could be dissolved into solution by allowing more time for the dissolution to occur, but that when the reactor was heated up to reaction temperatures there was no apparent effect on hydrolysis rates or extent.

\title{
Introduction
}

Previously published results showed a large degree of scatter in the data for Carbonic acid pretreatment of aspen wood. Further experiments were carried out to improve the data set and increase confidence in the results. With the increased number of data, a Q test at $99 \%$ certainty was used to eliminate outlier points and thus tighten up the results.

Through our studies of $\mathrm{H}_{2} \mathrm{CO}_{3}$ pretreatment of biomass, it has become apparent that there may be kinetic limitations affecting the consistency of hydrolysis results. A set of experiments was carried out to determine if the kinetics of solubilization of the $\mathrm{CO}_{2}$ was affecting hydrolysis. A reactor was charged with a xylan solution and $\mathrm{CO}_{2}$ at 800 psi. The pressurized reactor was then allowed to sit for a set period of time to allow mass transfer of the $\mathrm{CO}_{2}$ into solution prior to heating and initiation of the reaction.

\section{Materials and Methods}

Apparatus and materials: A $15 \mathrm{~mL}$ reactor, $150 \mathrm{~mL}$ reactor, sand bath, a coffee grinder, raw aspen wood, a sieve, a balance, deionized water, $\mathrm{pH}$ meter, a tank of carbon dioxide (lab grade), beakers, graduated cylinders, glass vials, and a spectrophotometer with quartz cuvettes.

\section{Procedures:}

Feedstock preparation: Raw pieces of aspen wood are placed into a coffee grinder. After the coffee grinder turned the wood into fine chips, the chips are placed into a sieve and shaken. The desired size of the wood was $1 \mathrm{~mm}$, and was collected for storage.

Aspen wood Pretreatment: The wood was taken to a balance and 0.1 grams is weighed. The 0.1 grams of wood was placed into a $15 \mathrm{~mL}$ reactor along with $8 \mathrm{~mL}$ of deionized water. For reactions involving carbon dioxide, 800 psi of carbon dioxide was added through a 1/8 inch stainless steel tube. The reactors are closed and placed in a sandbath. Some reactors were reacted at 180 Celsius and some at 200 Celsius. The amount of time that each reactor was placed in the sandbath was 8 minutes, 16 minutes, and 32 minutes. For repetition of older 
results, no pre-heat time was used to shorten temperature transients. This was because some of the earlier reported results were generated without pre-heat times.

Post reaction $\mathrm{pH}$ : After the reaction, the reactor was taken out and the hydrolysate emptied into a container such as a beaker. The $\mathrm{pH}$ was quickly measured. A magnetic stirrer bar was placed into the beaker and the hydrolysate was stirred in order to accelerate de-gassing. The $\mathrm{pH}$ was measured every 5 minutes until constant $\mathrm{pH}$ was attained.

UV-absorption: 30 microliters of the hydrolysate was placed into a micro-centrifuge tube and centrifuged for 3 minutes at 12,000 rpm. The hydrolysate was then pipetted into a quartz cuvette with 2970 microliters of water. The cuvette was placed into a spectrophotometer and the ultraviolet absorbance at $270 \mathrm{~nm}$ was measured.

Carbohydrates: Were measured by HPAE-PAD using a Dionex GP50 gradient pump, an ED40 amperometric detector and a Carbopac column running an eluent of dilute $\mathrm{NaOH}$.

Mass transfer experiments: Reactors were charged with aspen wood or xylan and water. The reactors were then pressurized with $\mathrm{CO}_{2}$ and then treated in one of three methods: 1 ) the reactors were sealed and immediately immersed in the sand bath to initiate reaction; 2)the reactors were sealed and allowed to sit idle at room temperature for a specific period of time: 15 minutes to 24 hours prior to reaction--this allowed the solution to become saturated with $\mathrm{CO}_{2}$ before reaction; and 3) the reactors were kept pressurized with $\mathrm{CO}_{2}$ for an hour before sealing the reactor, which allowed even more $\mathrm{CO}_{2}$ to go into solution, after which the reaction was then reacted. Conditions 1 and 2 were intended to compare the effects of saturation prior to reaction, while condition 3 allowed an even higher degree of saturation with $\mathrm{CO}_{2}$. Hydrolysates were analyzed for $\mathrm{pH}$, absorbance and xylose concentration.

\section{Results}

Tables 2.1 and 2.2 lists previous (McWilliams and van Walsum[1]) and newly generated (this study) results for aspen wood pretreatment experiments. As can be seen from the table, in most cases mean values from previous experiments have been confirmed and the error about the mean reduced. These same data are also plotted in figures $2.1-2.14$. On most of these figures it can be seen that the more recent results show less variation. Figure 2.15 shows the combined, averaged results from McWilliams and van Walsum[1] and the additional points generated in this study.

Results from experiments investigating the effect of dissolving time on xylan hydrolysis are found in tables 2.3 and 2.4. Figures 2.16 and 2.17 show the same data plotted versus dissolving time. Figure 2.18 shows xylose concentrations measured in response to three different conditions for dissolving time: no extra time for dissolution, three hours for dissolving, and 800 psi of $\mathrm{CO}_{2}$ pressure maintained during one hour prior to sealing the reactor. These three conditions represent progressively stronger conditions for dissolving $\mathrm{CO}_{2}$ prior to reaction. Although there appears to be a downward trend in the xylose concentration as solubilization intensity increases, the scatter in the repeats of each condition suggest that this may be arbitrary and not related to the degree of pre-reaction solubilization of $\mathrm{CO}_{2}$. If the trend is valid, then it appears to function in the opposite, and less easily explained, direction than that indicated by the apparent trend in UV absorption. 
Figure 2.19 shows results from increasing $\mathrm{CO}_{2}$ pressure applied to the reactor when hydrolyzing xylan. It can be seen that up to an initial pressure of 200 psi the additional $\mathrm{CO}_{2}$ offers advantages for hydrolysis. Above 200 psi the benefits of extra $\mathrm{CO}_{2}$ diminish.

Table 2.1. Comparison of Previous (McWilliams) and Recent (Lam) pH and UV Absorbance of Aspen Wood Hydrolysate Pretreated with Hot Carbonic Acid.

\begin{tabular}{|c|c|c|c|c|c|}
\hline & McWilliams & Lam & & McWilliams & Lam \\
\hline $\mathrm{H} 2 \mathrm{O}, \mathrm{CO} 2,180 \mathrm{C}, 16 \mathrm{~min}$ & $\mathrm{pH}$ & $\mathrm{pH}$ & & UV abs & UV abs \\
\hline 1 & 4.89 & 4.03 & 1 & 0.134 & 0.118 \\
\hline 2 & 3.54 & 4.39 & 2 & 0.243 & 0.147 \\
\hline 3 & 3.89 & 3.76 & 3 & 0.133 & 0.127 \\
\hline 4 & 4.04 & 4.04 & 4 & 0.132 & 0.175 \\
\hline 5 & & 3.79 & 5 & & 0.186 \\
\hline mean & 4.090 & 4.002 & & 0.1605 & 0.1506 \\
\hline std deviation & 0.496 & 0.226 & & 0.048 & 0.026 \\
\hline$\%$ std dev/mean & 12.1 & 5.7 & & 29.7 & 17.5 \\
\hline \multicolumn{6}{|l|}{$\mathrm{H} 2 \mathrm{O}, \mathrm{CO} 2,180 \mathrm{C}, 32 \mathrm{~min}$} \\
\hline 1 & 4.34 & 3.7 & 1 & 0.197 & 0.197 \\
\hline 2 & 3.75 & 3.97 & 2 & 0.325 & 0.213 \\
\hline 3 & 3.9 & 4.17 & 3 & 0.144 & 0.152 \\
\hline 4 & 4.42 & 3.68 & 4 & 0.122 & 0.216 \\
\hline 5 & & 3.93 & & & \\
\hline mean & 4.103 & 3.890 & & 0.1970 & 0.1945 \\
\hline std deviation & 0.284 & 0.183 & & 0.079 & 0.026 \\
\hline$\%$ std dev/mean & 6.9 & 4.7 & & 40.0 & 13.2 \\
\hline \multicolumn{6}{|l|}{$\mathrm{H} 2 \mathrm{O}, \mathrm{CO} 2,200 \mathrm{C}, 16 \mathrm{~min}$} \\
\hline $\begin{array}{ll} & 1\end{array}$ & 5.13 & 3.74 & 1 & 0.24 & 0.316 \\
\hline 2 & 3.78 & 3.69 & 2 & 0.451 & 0.221 \\
\hline 3 & 4.01 & 3.79 & 3 & 0.304 & 0.237 \\
\hline 4 & & 3.75 & 4 & & 0.228 \\
\hline mean & 4.307 & 3.743 & & 0.3317 & 0.2505 \\
\hline std deviation & 0.590 & 0.036 & & 0.088 & 0.038 \\
\hline$\%$ std dev/mean & 13.7 & 1.0 & & 26.6 & 15.3 \\
\hline
\end{tabular}


Table 2.2. Comparison of Previous (McWilliams) and Recent (Lam) pH and UV Absorbance of Aspen Wood Hydrolysate

Pretreated with Liquid Hot Water.

\begin{tabular}{|c|c|c|c|c|c|}
\hline & McWilliams & Lam & & McWilliams & Lam \\
\hline $\mathrm{H} 2 \mathrm{O}, 180 \mathrm{C}, 8 \mathrm{~min}$ & $\mathrm{pH}$ & $\mathrm{pH}$ & & UV abs & UV abs \\
\hline 1 & 4.29 & 3.76 & 1 & 0.087 & 0.122 \\
\hline 2 & 3.29 & 4.04 & 2 & 0.135 & 0.13 \\
\hline 3 & 4.25 & 3.79 & 3 & 0.076 & 0.085 \\
\hline 4 & 4.02 & 3.98 & 4 & 0.169 & 0.12 \\
\hline mean & 3.963 & 3.893 & & 0.1168 & 0.1143 \\
\hline std deviation & 0.402 & 0.120 & & 0.037 & 0.017 \\
\hline$\%$ std dev/mean & 10.1 & 3.1 & & 32.1 & 15.1 \\
\hline \multirow{2}{*}{\multicolumn{6}{|c|}{$\mathrm{H} 2 \mathrm{O}, 180 \mathrm{C}, 16 \mathrm{~min}$}} \\
\hline & & & & & \\
\hline 1 & 3.95 & 3.75 & 1 & 0.153 & 0.171 \\
\hline 2 & 3.22 & 3.61 & 2 & 0.185 & 0.189 \\
\hline 3 & 3.56 & 3.52 & 3 & 0.168 & 0.22 \\
\hline 4 & 3.78 & 3.63 & 4 & 0.139 & 0.166 \\
\hline mean & 3.628 & 3.628 & & 0.1613 & 0.1865 \\
\hline std deviation & 0.273 & 0.082 & & 0.017 & 0.021 \\
\hline$\%$ std dev/mean & 7.5 & 2.3 & & 10.6 & 11.3 \\
\hline \multicolumn{6}{|l|}{$\mathrm{H} 2 \mathrm{O}, 180 \mathrm{C}, 32 \mathrm{~min}$} \\
\hline 10 & 3.85 & 3.34 & 1 & 0.193 & 0.197 \\
\hline 2 & 3.34 & 3.35 & 2 & 0.443 & 0.213 \\
\hline 3 & 3.56 & 3.67 & 3 & 0.19 & 0.152 \\
\hline 4 & 3.65 & 3.68 & 4 & 0.179 & 0.216 \\
\hline mean & 3.600 & 3.510 & & 0.2513 & 0.1945 \\
\hline std deviation & 0.183 & 0.165 & & 0.111 & 0.026 \\
\hline$\%$ std dev/mean & 5.1 & 4.7 & & 44.1 & 13.2 \\
\hline \multicolumn{6}{|l|}{$\mathrm{H} 2 \mathrm{O}, \mathrm{CO} 2,180 \mathrm{C}, 8 \mathrm{~min}$} \\
\hline 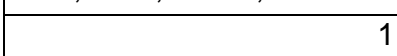 & 4.7 & 4.17 & 1 & 0.091 & 0.111 \\
\hline 2 & 3.62 & 4.36 & 2 & 0.456 & 0.114 \\
\hline 3 & 4.23 & 4.36 & 3 & 0.099 & 0.106 \\
\hline 4 & 4.29 & 4.74 & 4 & 0.092 & 0.025 \\
\hline 5 & & 5.16 & 5 & & 0.107 \\
\hline mean & 4.047 & 4.655 & & 0.2157 & 0.0880 \\
\hline std deviation & 0.303 & 0.330 & & 0.170 & 0.037 \\
\hline$\%$ std dev/mean & 7.5 & 7.1 & & 78.8 & 41.5 \\
\hline
\end{tabular}


Figure 2.1

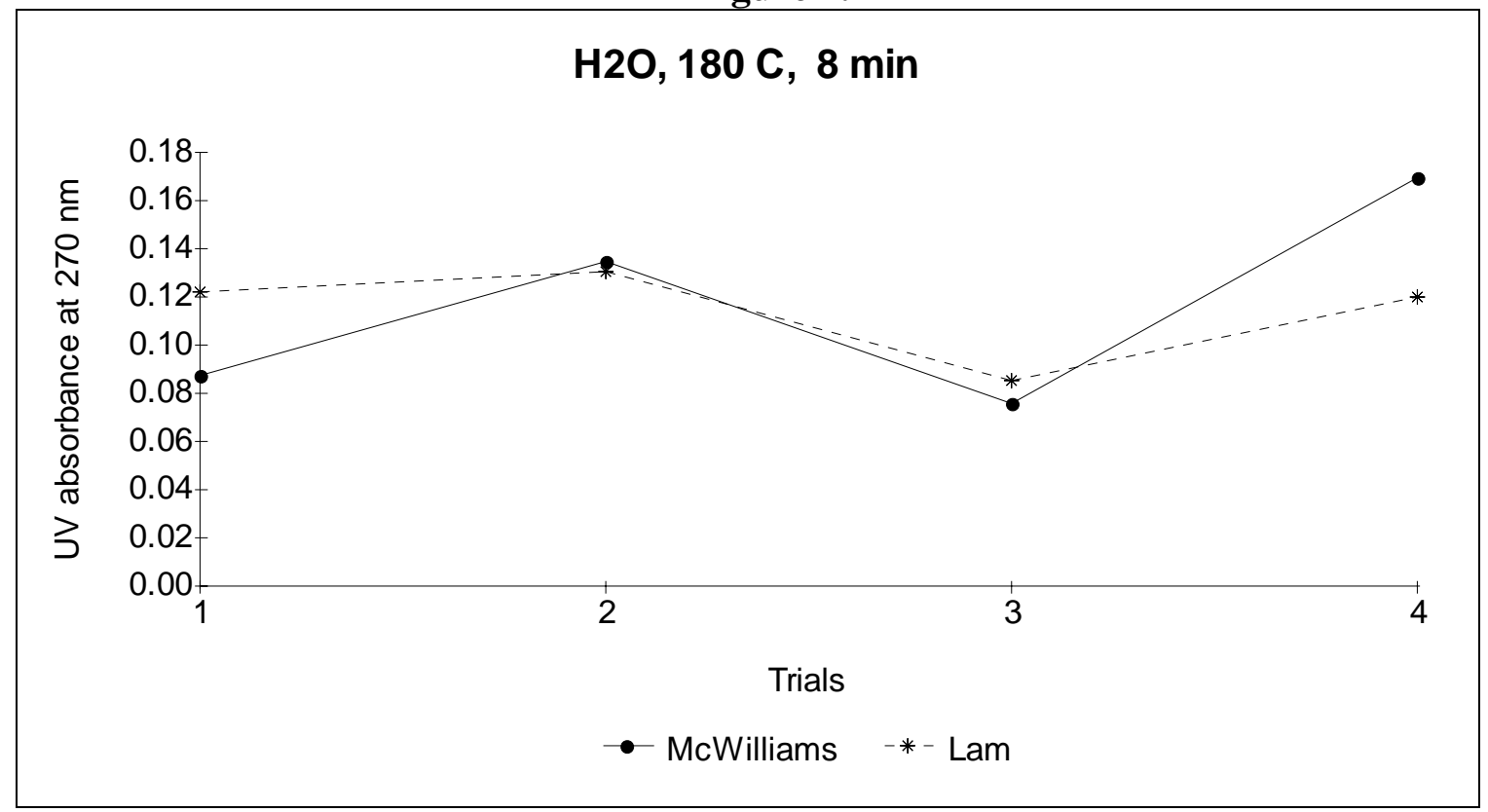

Figure 2.2

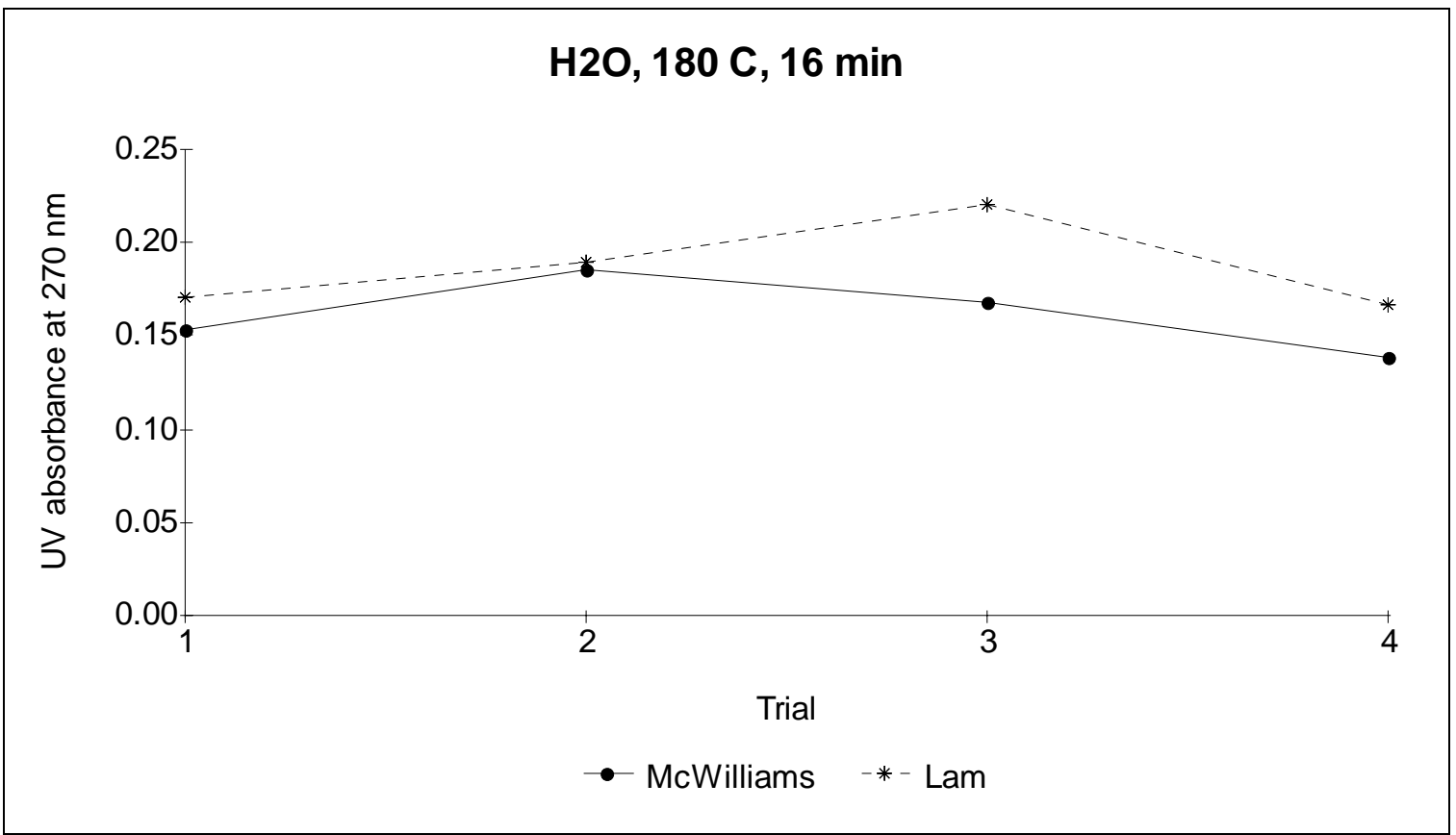


Figure 2.3

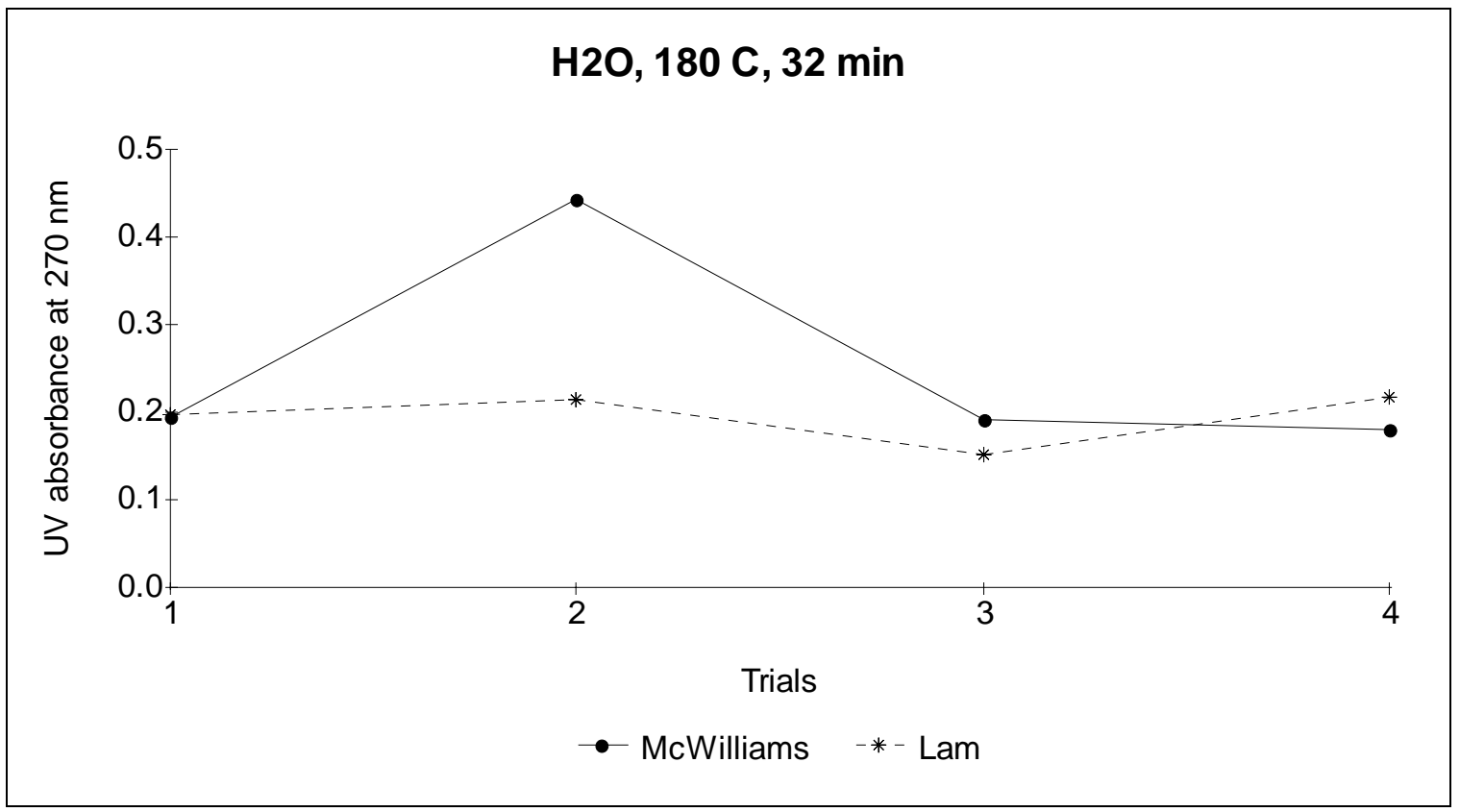

Figure 2.4

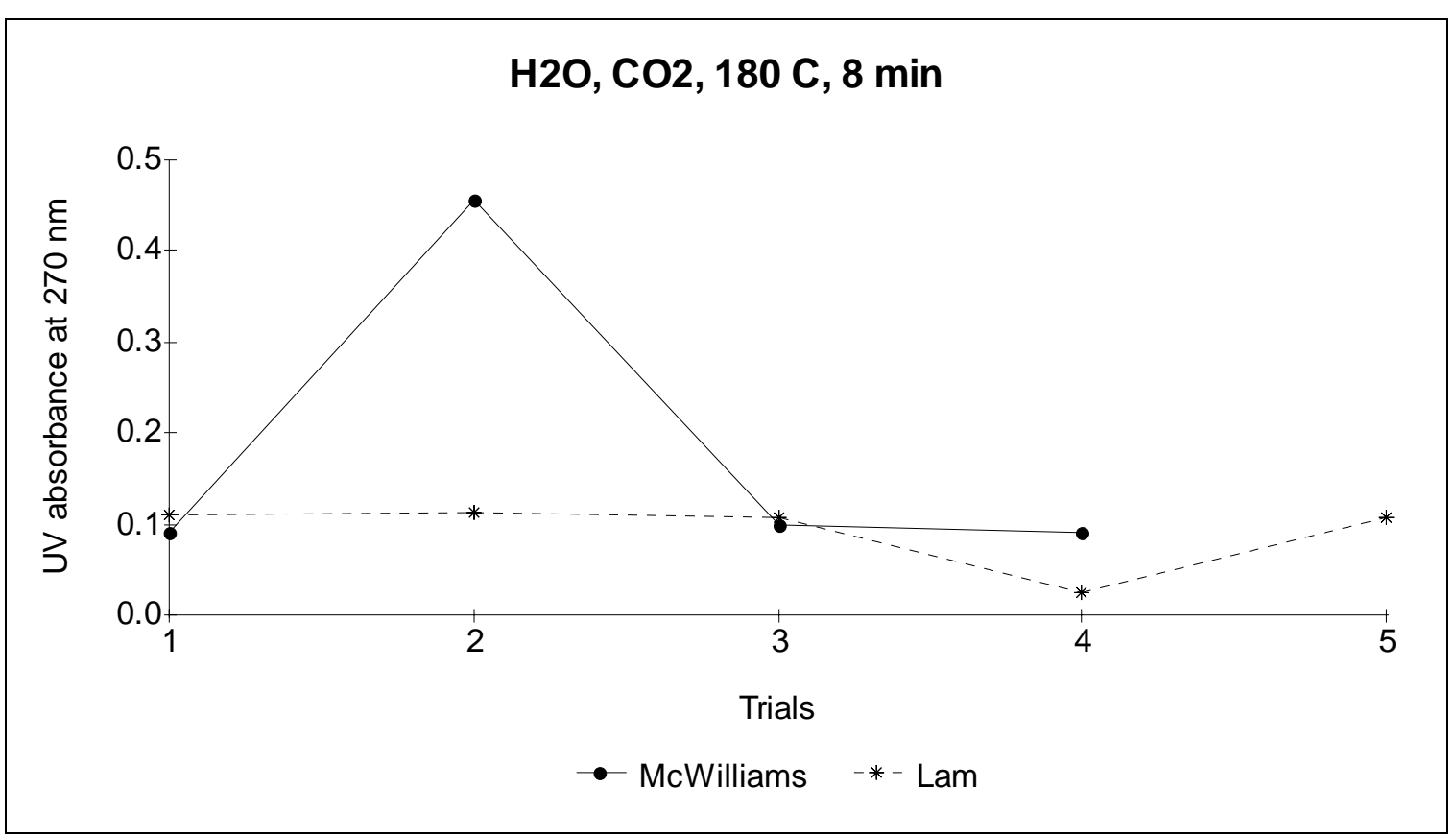


Figure 2.5

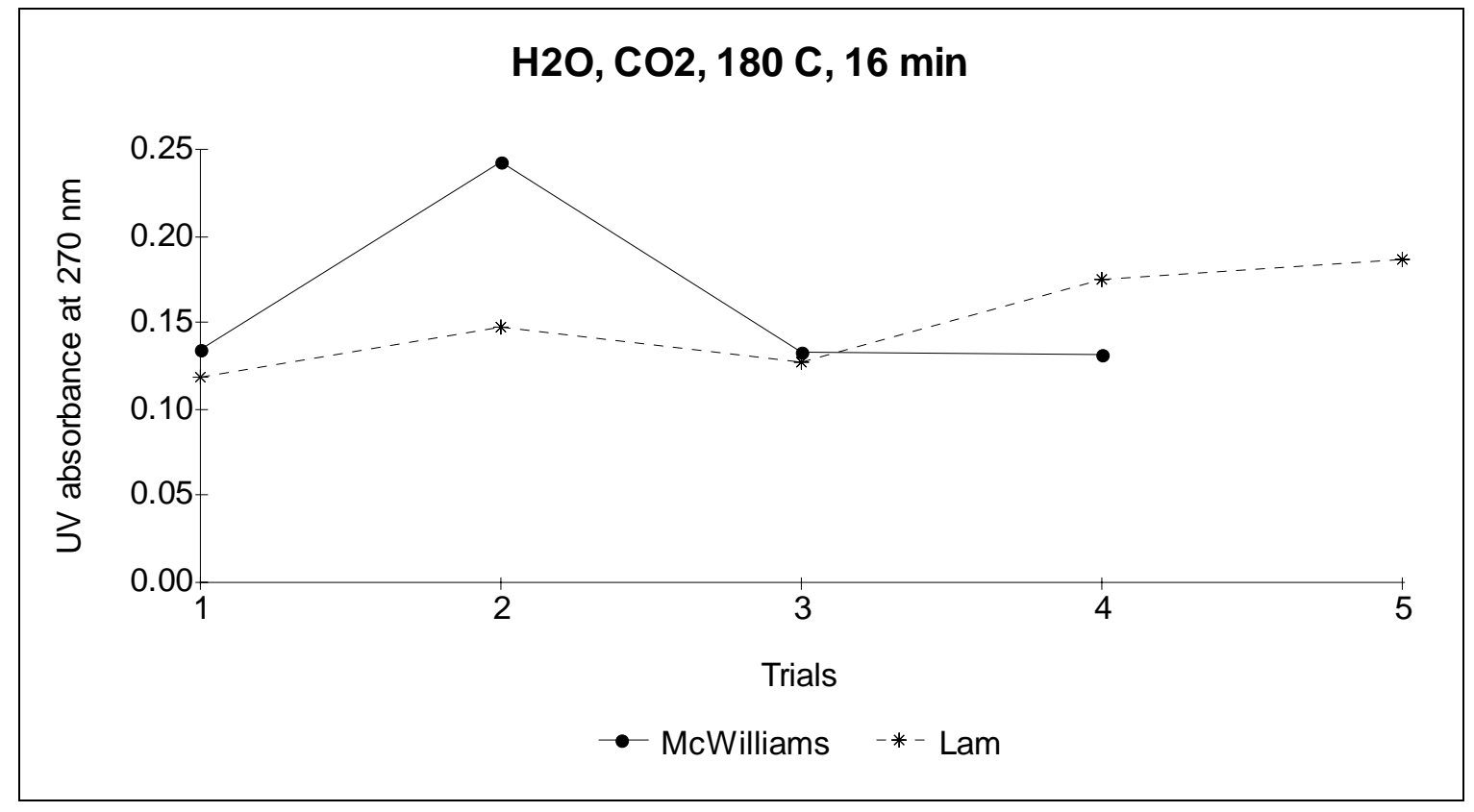

Figure 2.6

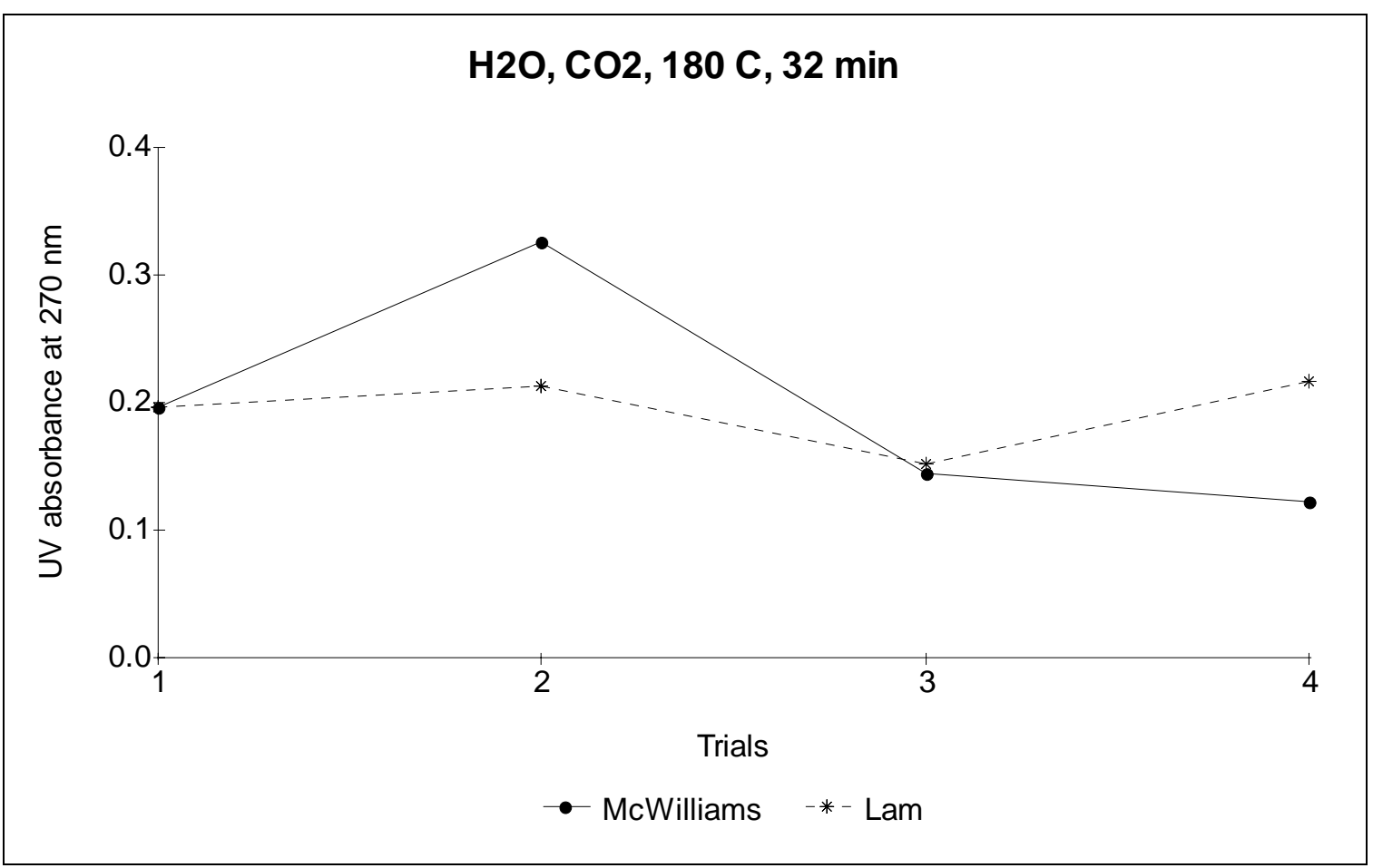

Figure 2.7 


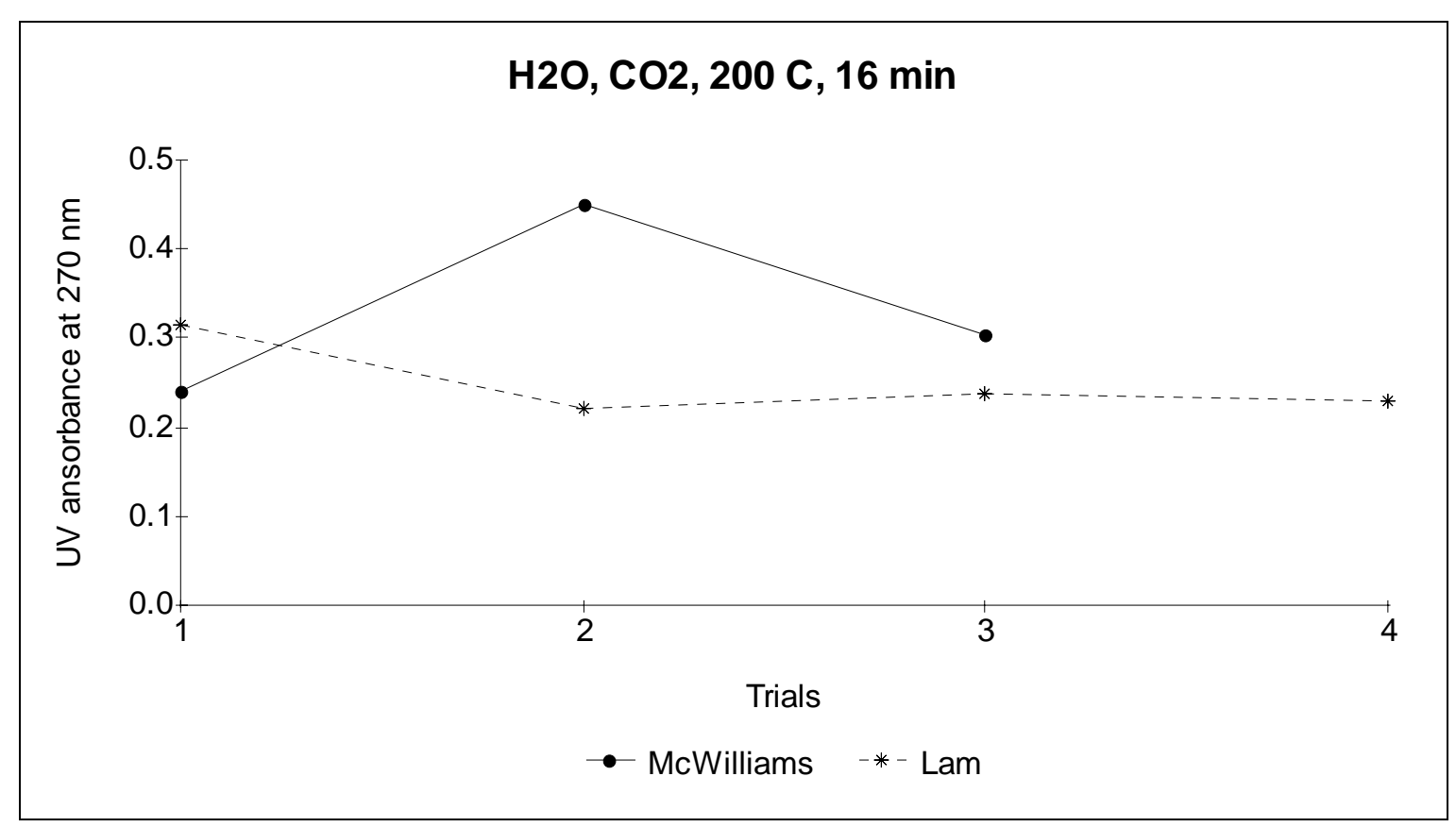

Figure 2.8

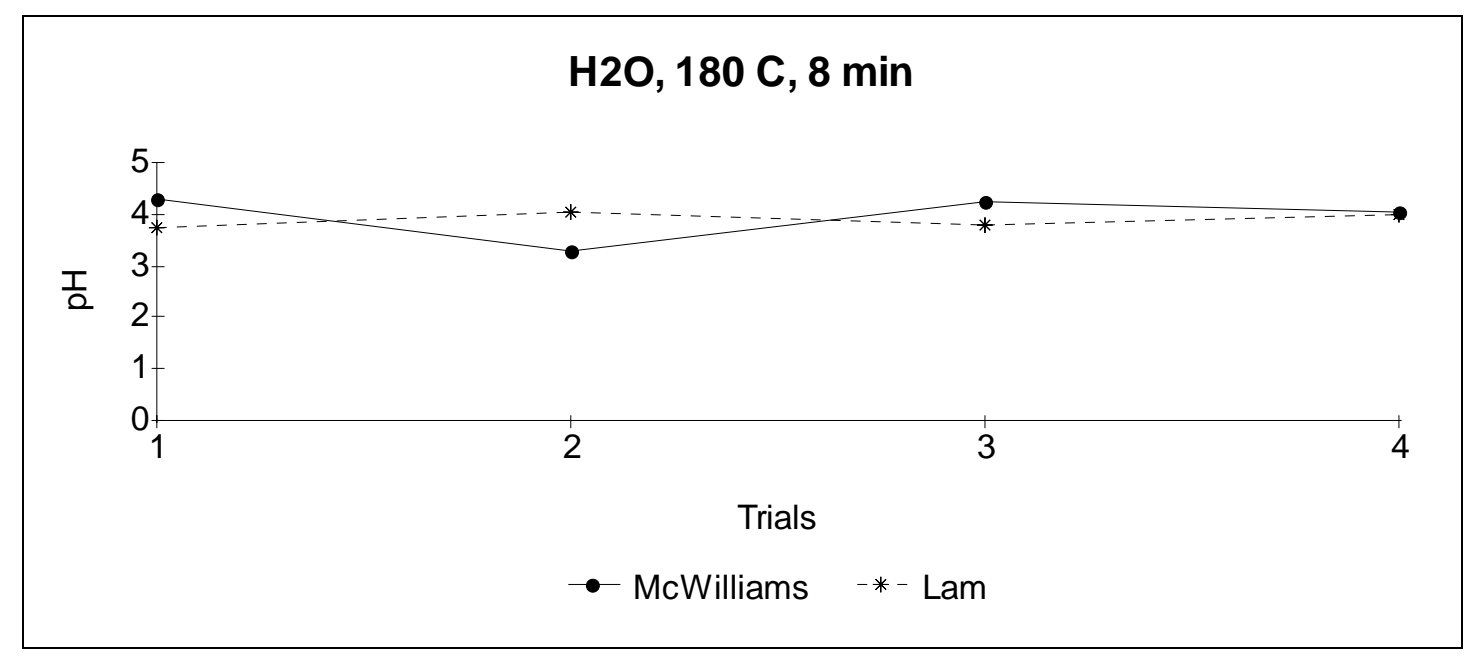


Figure 2.9

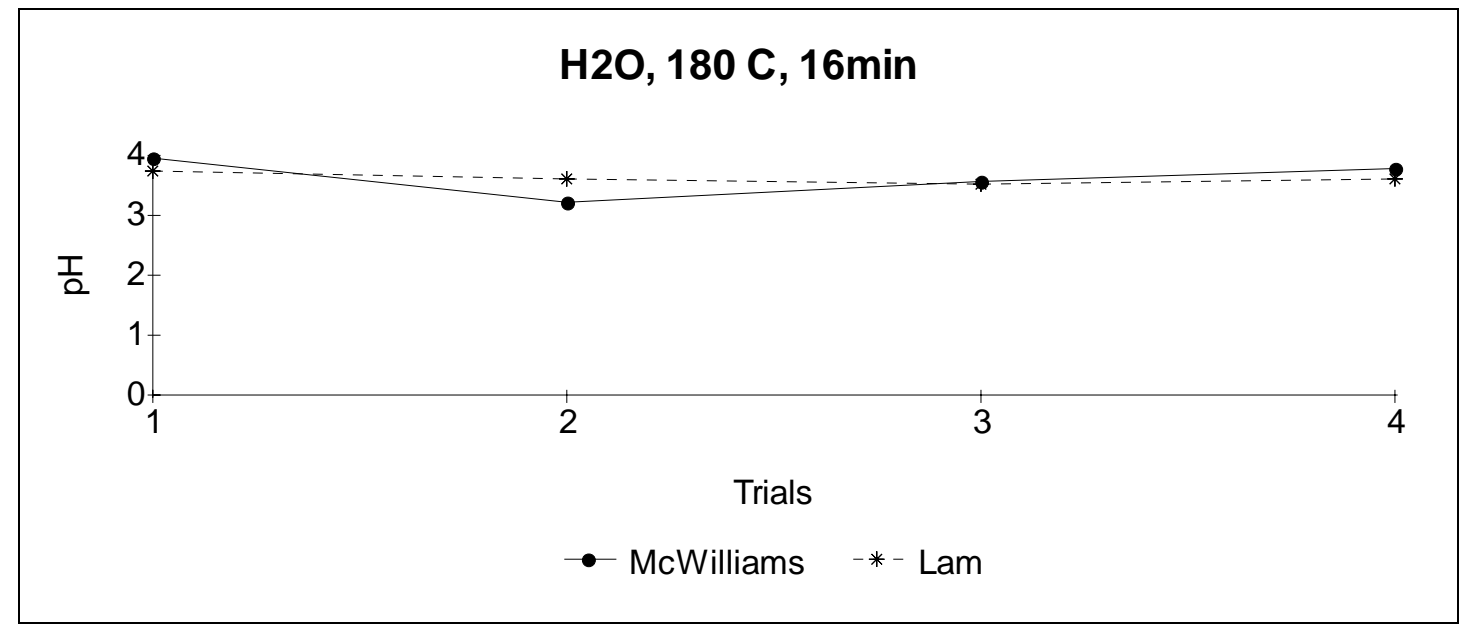

Figure 2.10

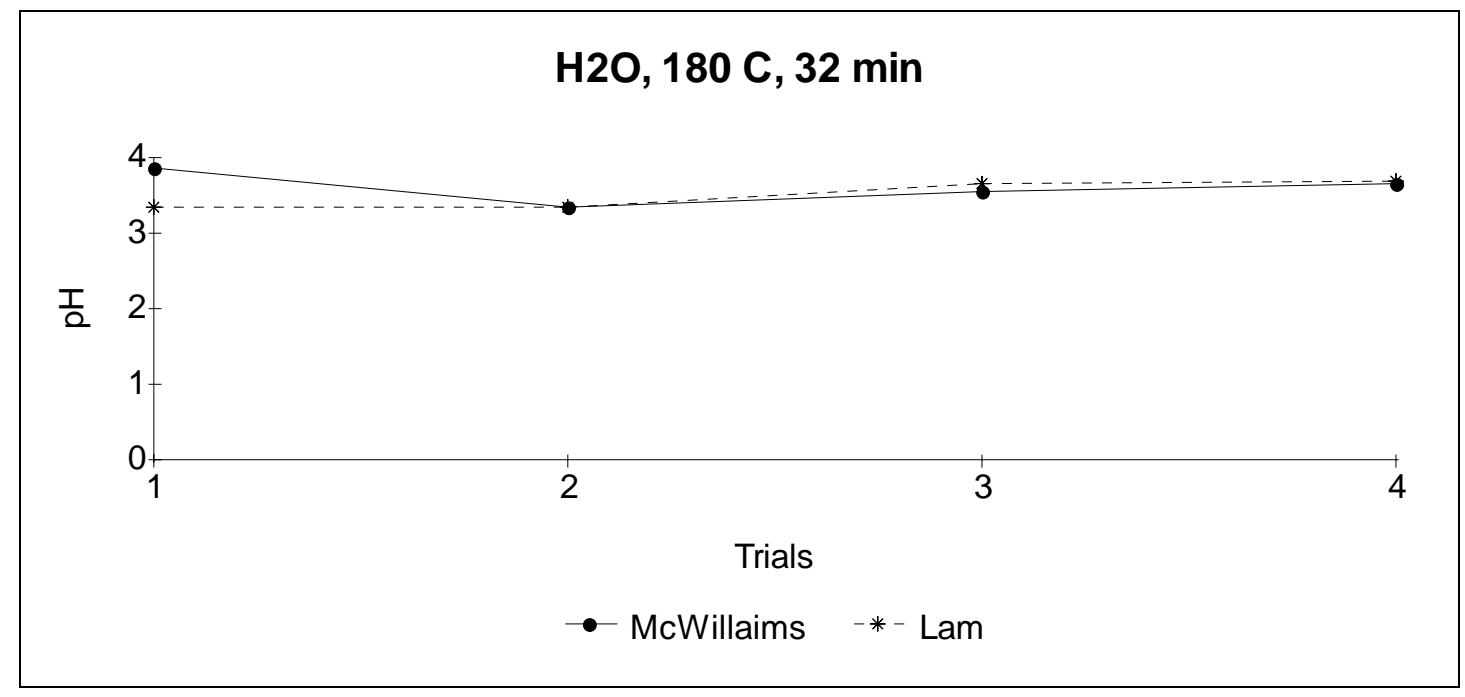


Figure 2.11

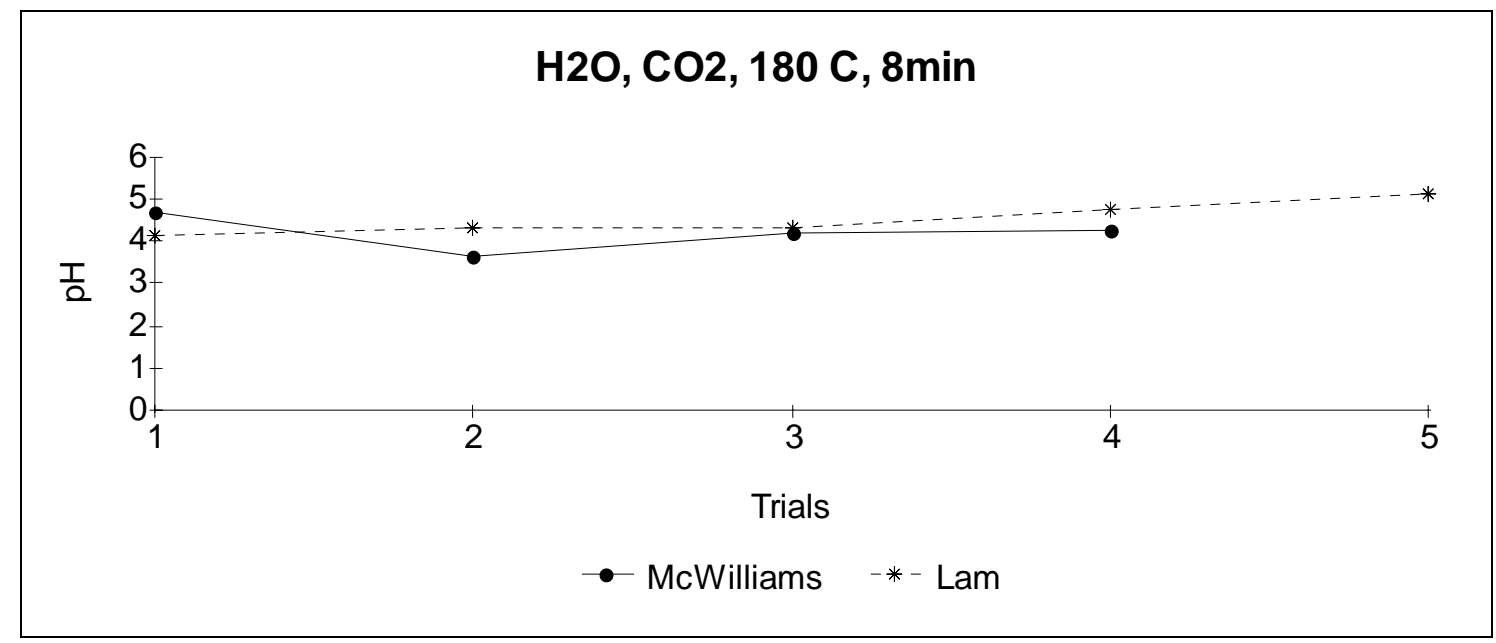

Figure 2.12

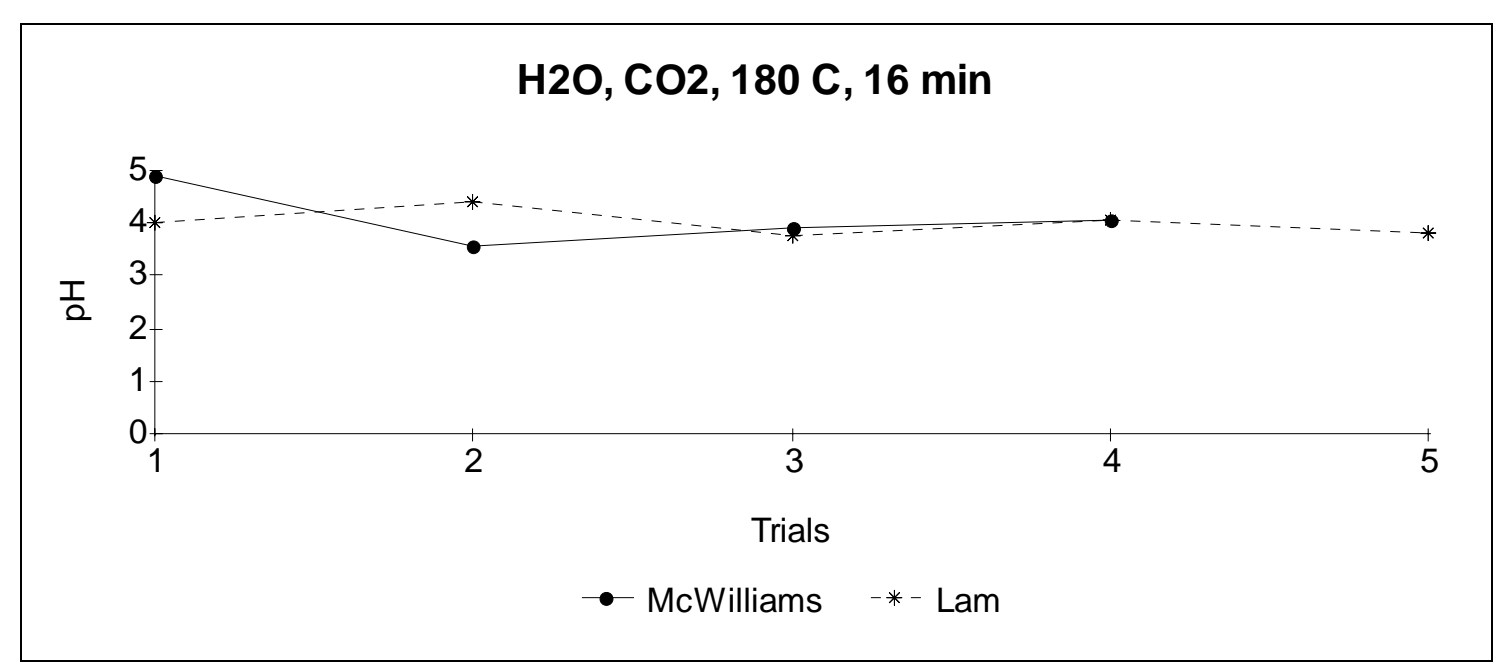


Figure 2.13

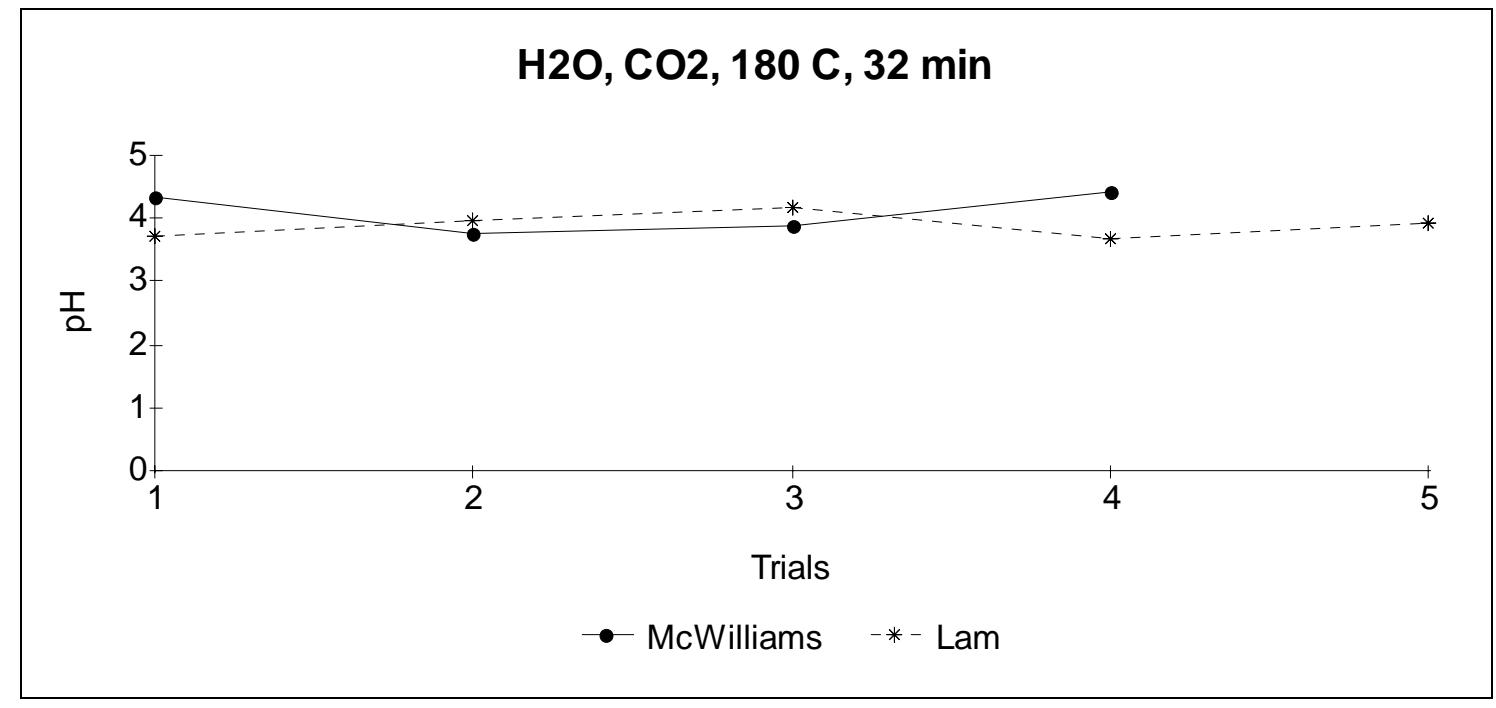

Figure 2.14

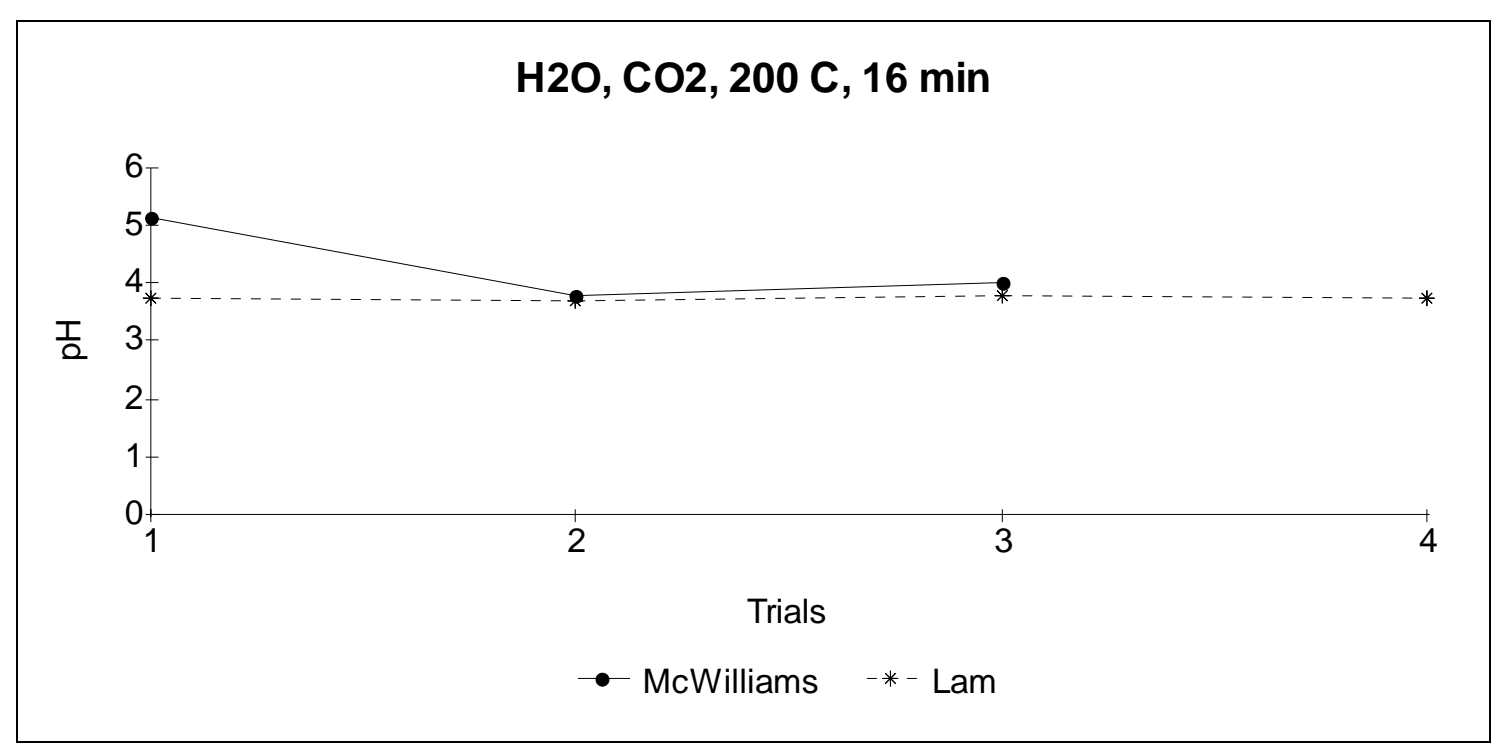


Figure 2.15 Response of UV absorbance, final pH and xylose concentrations versus reaction severity for Carbonic Acid Pretreatment of Aspen Wood: combined results from McWilliams and van Walsum [1] and this study

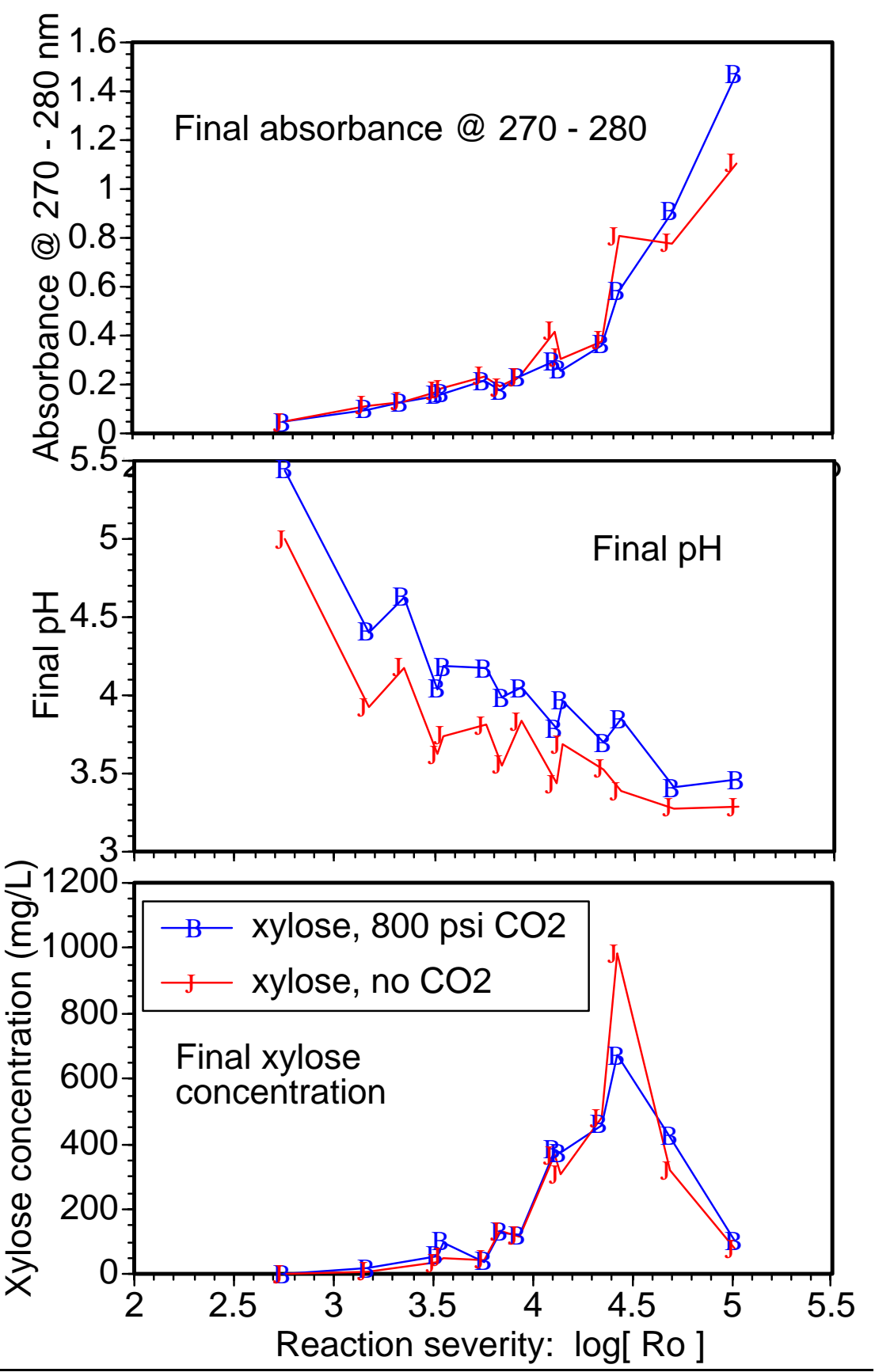


Table 2.3 pH and Absorbance Results for Varying Pre-reaction Solubilization Time

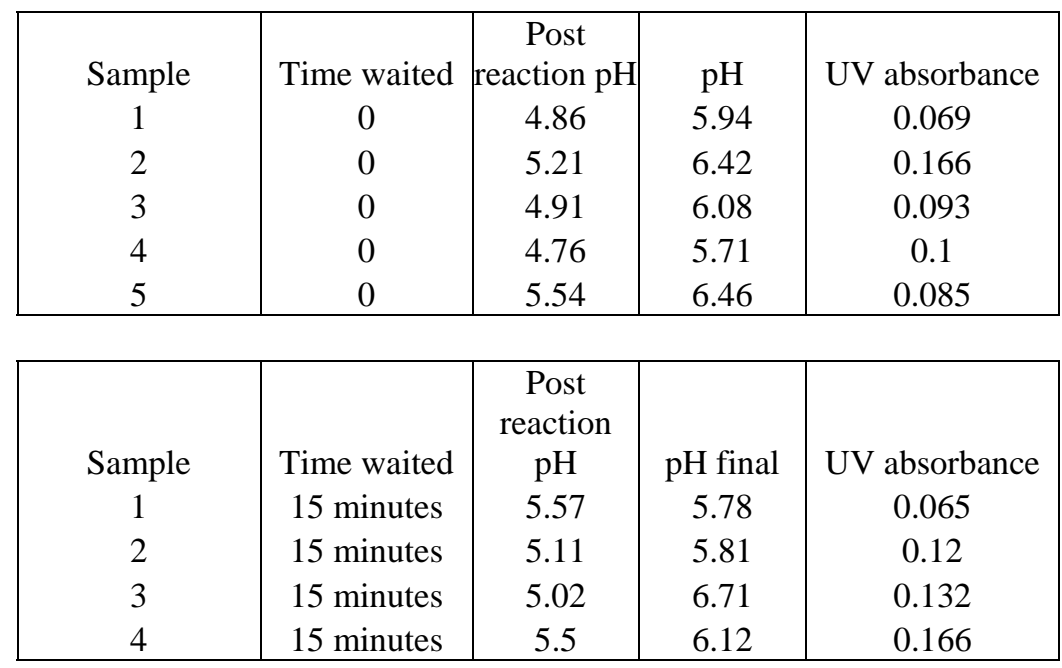

\begin{tabular}{|c|c|c|c|c|}
\hline & & Post & & \\
Sample & Time waited & reaction $\mathrm{pH}$ & $\mathrm{pH}$ final & UV absorbance \\
1 & 30 minutes & 4.86 & 6.26 & 0.13 \\
2 & 30 minutes & 4.91 & 6.77 & 0.102 \\
\hline
\end{tabular}

\begin{tabular}{|c|c|c|c|c|}
\hline & & Post & & \\
Sample & Time waited & reaction $\mathrm{pH}$ & $\mathrm{pH}$ final & UV absorbance \\
1 & 5 hours & 5.51 & 6.44 & 0.166 \\
\hline
\end{tabular}

\begin{tabular}{|c|c|c|c|c|}
\hline Sample & Time waited & $\begin{array}{c}\text { Post } \\
\text { reaction } \mathrm{pH}\end{array}$ & $\mathrm{pH}$ final & UV absorbance \\
1 & 24 hours & 5.24 & 6.35 & 0.141 \\
2 & 24 hours & 5.32 & 6.4 & 0.126 \\
3 & 24 hours & 5.14 & 6.11 & 0.109 \\
4 & 24 hours & 5.35 & 6.65 & 0.161 \\
\hline
\end{tabular}


Figure $2.16 \mathrm{pH}$ values versus Solubilization time

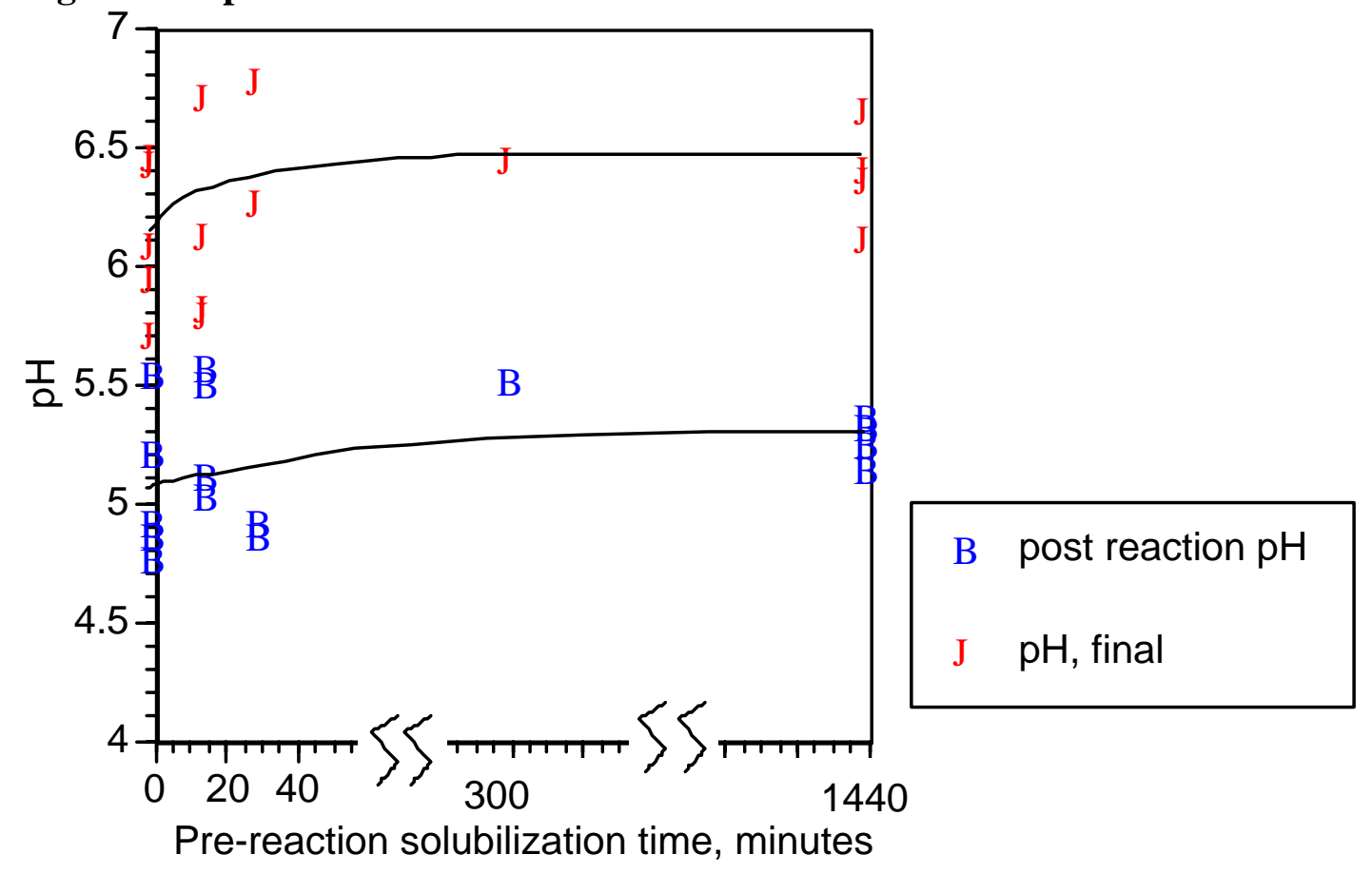

Figure 2.17 UV absorption vs Solubilization time

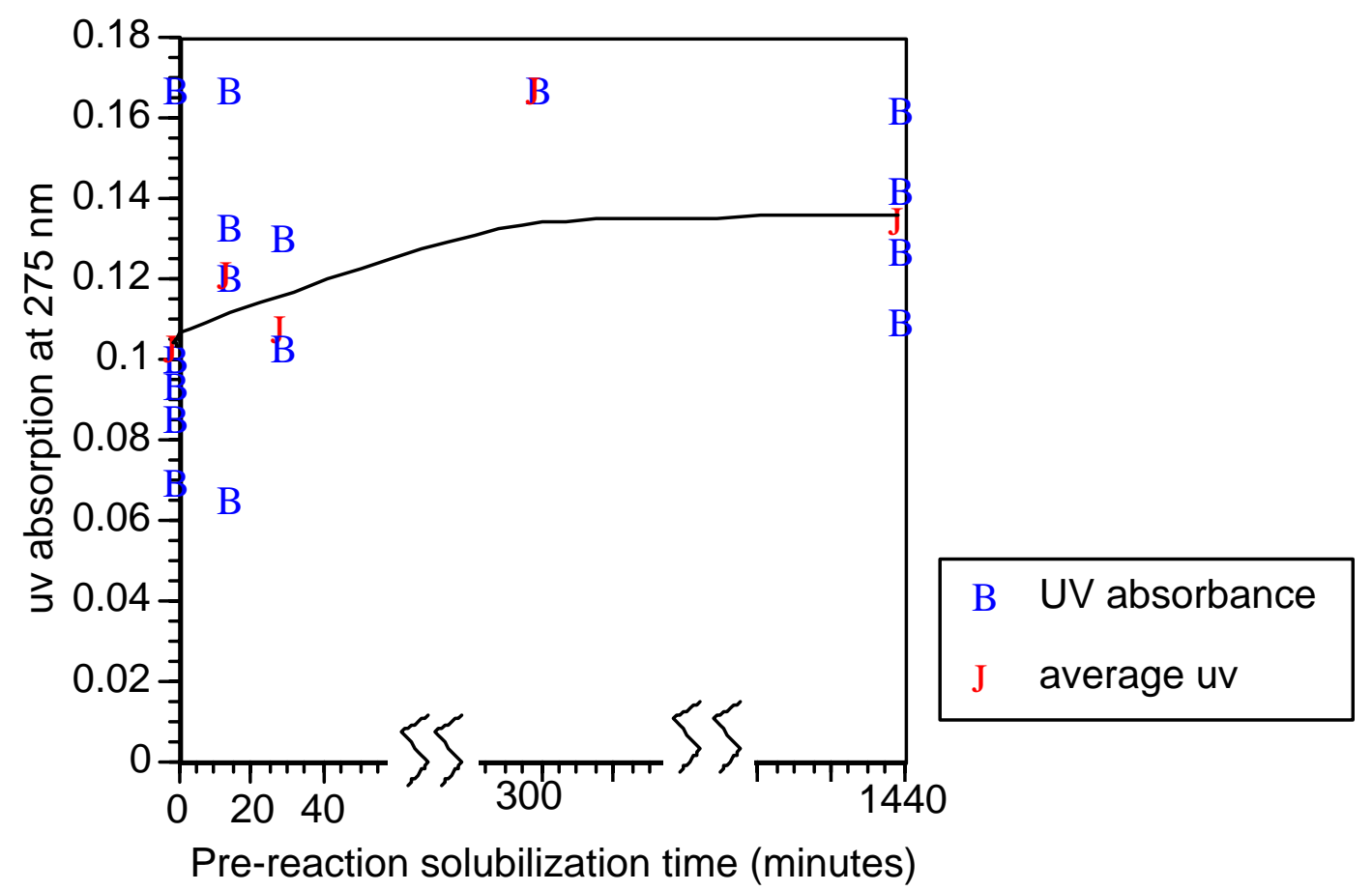


Figure 2.18 Effect on xylose concentration of increasing $\mathrm{CO}_{2}$ solubilization prior to reaction.

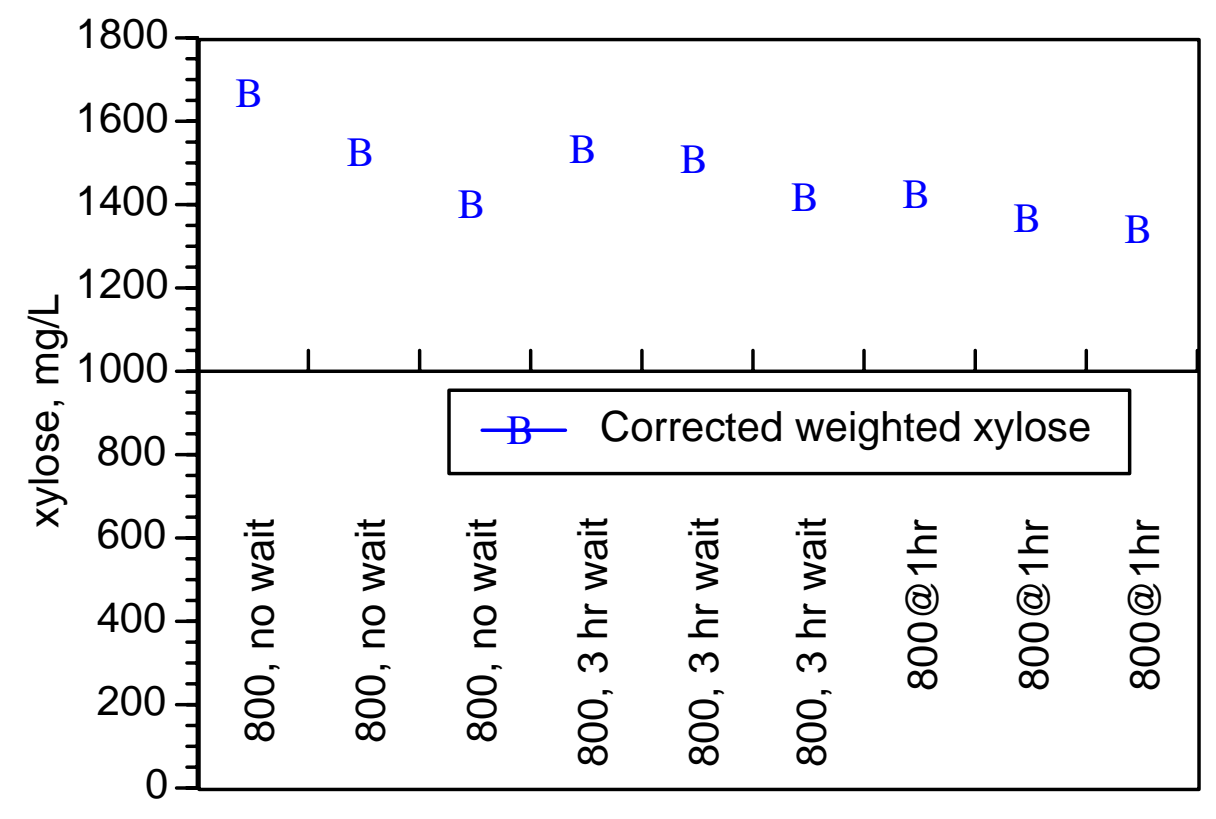

Figure 2.19: Response of xylose accumulation to increasing initial CO2 pressure.

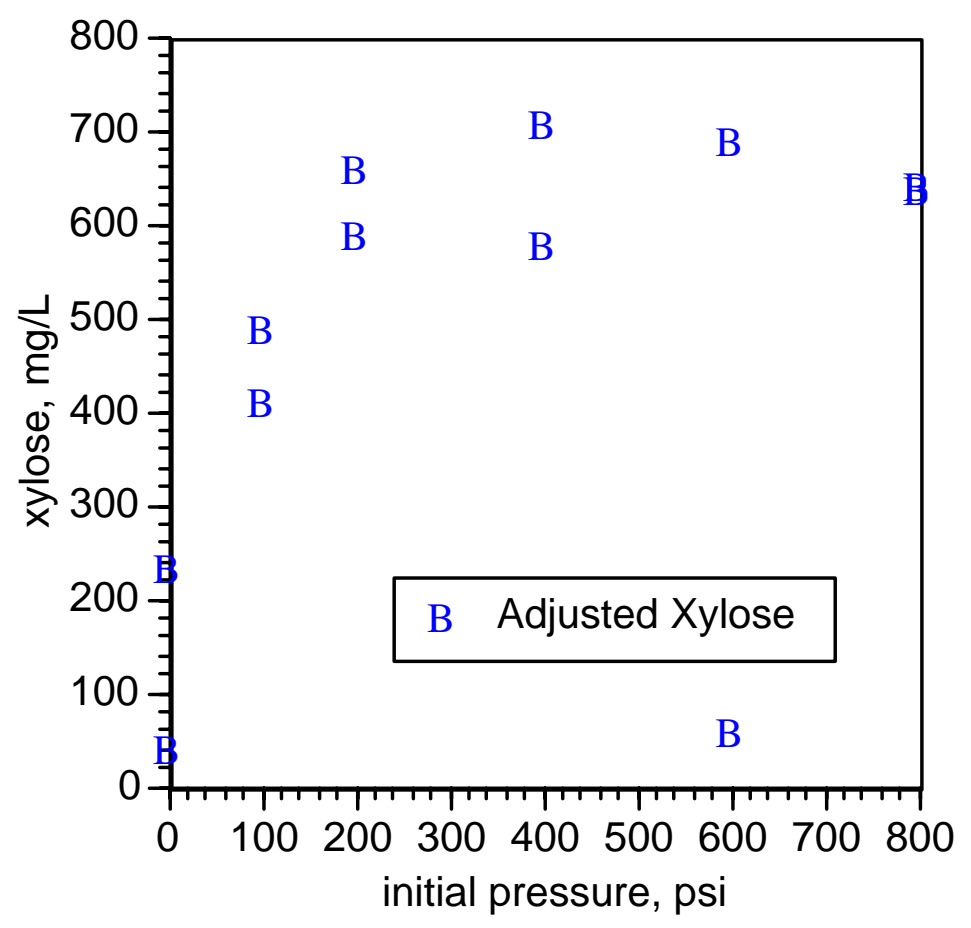




\section{Discussion}

Tables 2.1 and 2.2 show how previous results have been confirmed and the accuracy of the experiment improved. This improvement is encouraging, since the final results include experiments carried out by three different researchers, over a period of more than one yearillustrating good reliability for the methods. Of the original data collection, the UV absorbance data were the most erratic. Repeat experiments have improved upon the $\mathrm{pH}$ data and have in some cases dramatically improved the UV absorption data. In most cases, the large error reported in the initial findings were due to extreme outlying points. Statistically. it is now possible to justify dropping these severe outlying points with a high degree of certainty.

For the mass transfer experiments, scatter in the data do prevent drawing any firm conclusions at this point. There does appear to be a mild trends associated with longer periods of time allowed for solubilization of the $\mathrm{CO}_{2}$, but these trends appear to move in opposite directions for UV absorbance and xylose production, and thus are likely figments of the scatter in the data. Issues that still ought to be addressed with respect to this investigation are the amount of time needed to achieve equilibrium concentrations and whether or not super saturation of the solution is occurring in the reaction, since this could allow more acidic conditions than predicted by thermodynamic predictions of the equilibrium state of the system.

Results in figure 2.19, plotting xylose production versus $\mathrm{CO}_{2}$ pressure are similar to those published by van Walsum in 2001[2], although van Walsum found a more continuous response to increased pressure throughout the range. It is possible that the quantity of xylose released in this more recent study was reaching a maximum due to completion of hydrolysis, as opposed to saturation with acid. However, if the observed result is more robust than this, it implies that carbonic acid pretreatment may be effective at lower pressures than currently used for most investigations. Because high pressures are expensive to implement in process conditions, this point deserves further exploration.

\section{Conclusion}

It appears that increased availability of $\mathrm{CO}_{2}$ increases hydrolysis, but that saturation of the system prior to reaction has relatively little effect on hydrolysis. This is likely caused by the $\mathrm{CO}_{2}$ coming out of solution as the reactor is heated up to reaction temperature. There may be a leveling off of hydrolysis enhancement at about $200 \mathrm{psi}$ initial pressure of $\mathrm{CO}_{2}$. This result should be confirmed because of its strong implications for reducing processing costs associated with high pressures.

\section{References}

[1] Robert C. McWilliams, G. Peter van Walsum, 2002. Comparison of Aspen Wood Hydrolysates Produced by Pretreatment with Liquid Hot Water and Carbonic Acid. Accepted for publication in Applied Biochemistry and Biotechnology

[2] Van Walsum, G. Peter, 2001. Severity Function Describing the Hydrolysis of Xylan Using Carbonic Acid. Applied Biuochemistry and Biotechnology, 91-93: 317 - 329 
TASK 3 Compare the results between hydrolysis of purified xylan and raw biomass.

\author{
John Lam, Robert McWilliams, G. Peter van Walsum
}

\title{
Summary
}

It was found that the severity function developed by Overend and Chornet [2] adequately described the action of time and temperature on the pretreatmentof aspen wood but not pure xylan. For aspen wood, no significant difference was detected between carbonic acid and water systems, thus the effect of the carbonic acid was negligible and did not need to be incorporated into the severity function. On xylan, however, it was necessary to take into account the action of the carbonic acid, and it was found that the severity function proposed by van Walsum[3] did so adequately. Published literature results for the dilute acid pretreatment of softwood $[4,5]$ were used for comparing the fit of the severity function and the combined severity function for lower $\mathrm{pH}$ systems. It was found that the literature data had considerable scatter, but that the combined severity function did appear to offer a more predictive capability than the regular severity function, which does not take into account the $\mathrm{pH}$ of the system.

\section{Results:}

Figure 3.1( same as 2.15, above) shows how absorbance, final $\mathrm{pH}$ and xylose yield respond to varying reaction severity. In this case, the severity is calculated without taking into account the $\mathrm{pH}$ of the system. Because the results from the liquid hot water system mirror those of the carbonic acid system, it appears that the $\mathrm{pH}$ of the carbonic acid has no significant effect on the degree of hydrolysis. Thus, in the case of aspen wood, it appears that the simple severity function is adequate to characterize the extent of reaction.

The same result was not found with xylan. Figure 3.2 (same as 2.19 above) shows how the extent of hydrolysis for xylan is strongly influenced by the pressure of $\mathrm{CO}_{2}$ in the reactor. In this case, the combined severity function proposed by van Walsum [3] was used to replot the data presented in figure3.2. In figure 3.3 it can be seen that the combined severity function does indeed offer a better characterization of the system than does the simple severity function, which for these data would have a single value.

Published results were used to asses the usefulness of the combined severity function at lower $\mathrm{pH}$ values. Reported values of dry matter remaining after hydrolysis were plotted versus the $\log$ of the severity function: $\log (\mathrm{Ro})$, and the combined severity function: $\mathrm{CS}=\log (\mathrm{Ro})-\mathrm{pH}$. Results are shown in figures 3.4 and 3.5. It can be seen that the general linearity of fit is better with the combined severity function, especially at higher severities. 
Figure 3.1 Response of UV absorbance, final $\mathrm{pH}$ and xylose concentrations versus reaction severity for Carbonic Acid Pretreatment of Aspen Wood: combined results from McWilliams and van Walsum [1] and task 2 of this study.

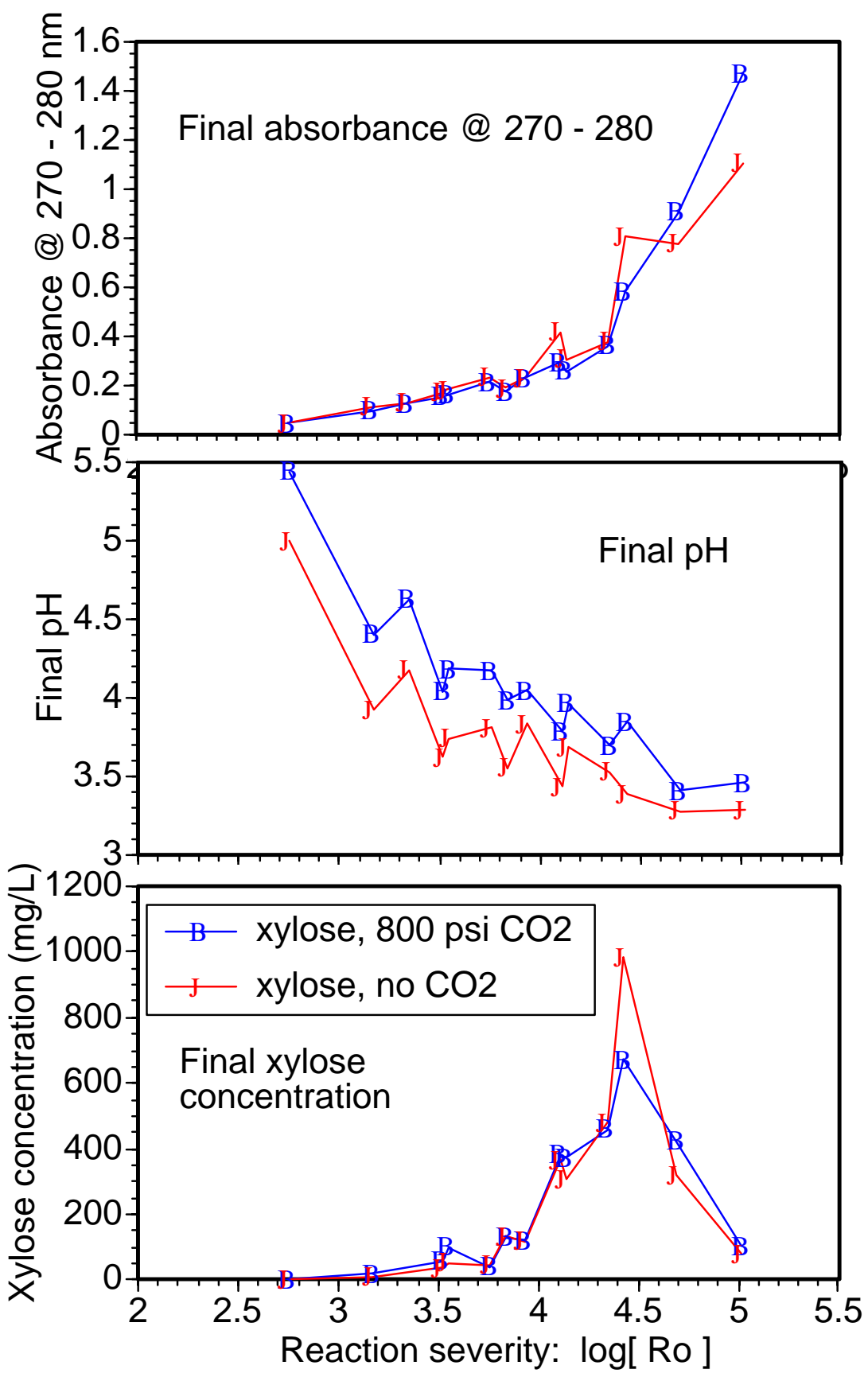


Figure3.2: Response of xylose accumulation to increasing initial $\mathrm{CO}_{2}$ pressure.

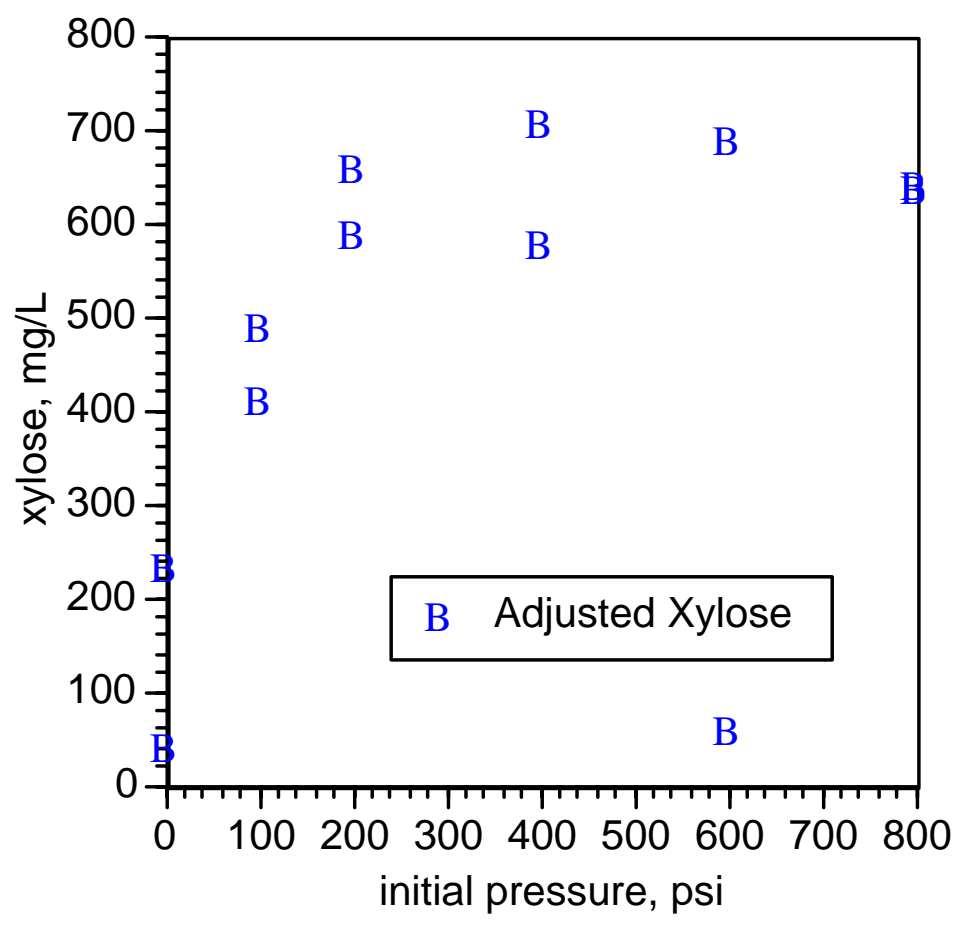

Figure 3.3 Xylose yield from hydrolysis of xylan at $190 \mathrm{C}$ for 16 minutes, with combined severity reflecting varying pressures of $\mathrm{CO}_{2}$.

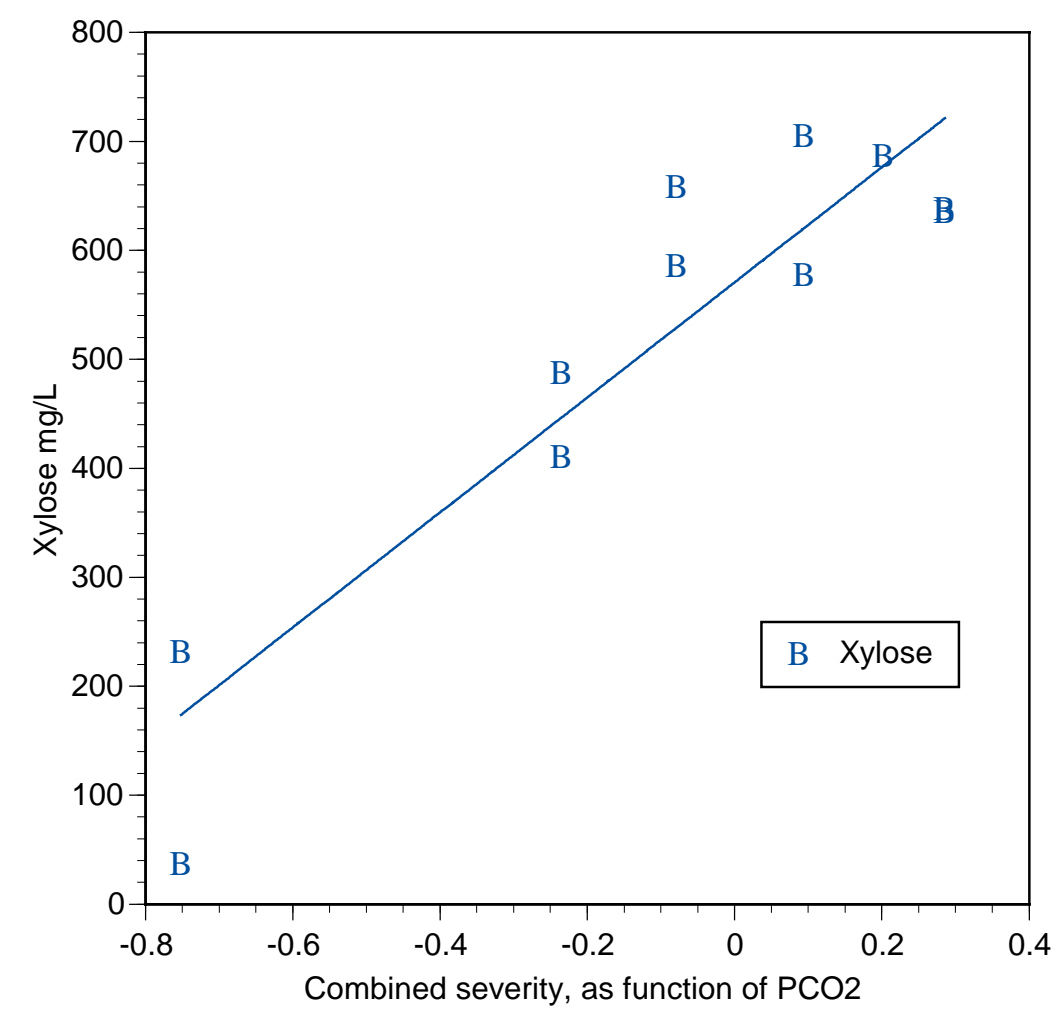

Figure 3.4 Residual dry matter versus combined severity for dilute sulfuric acid hydrolysis of pine wood [4] 


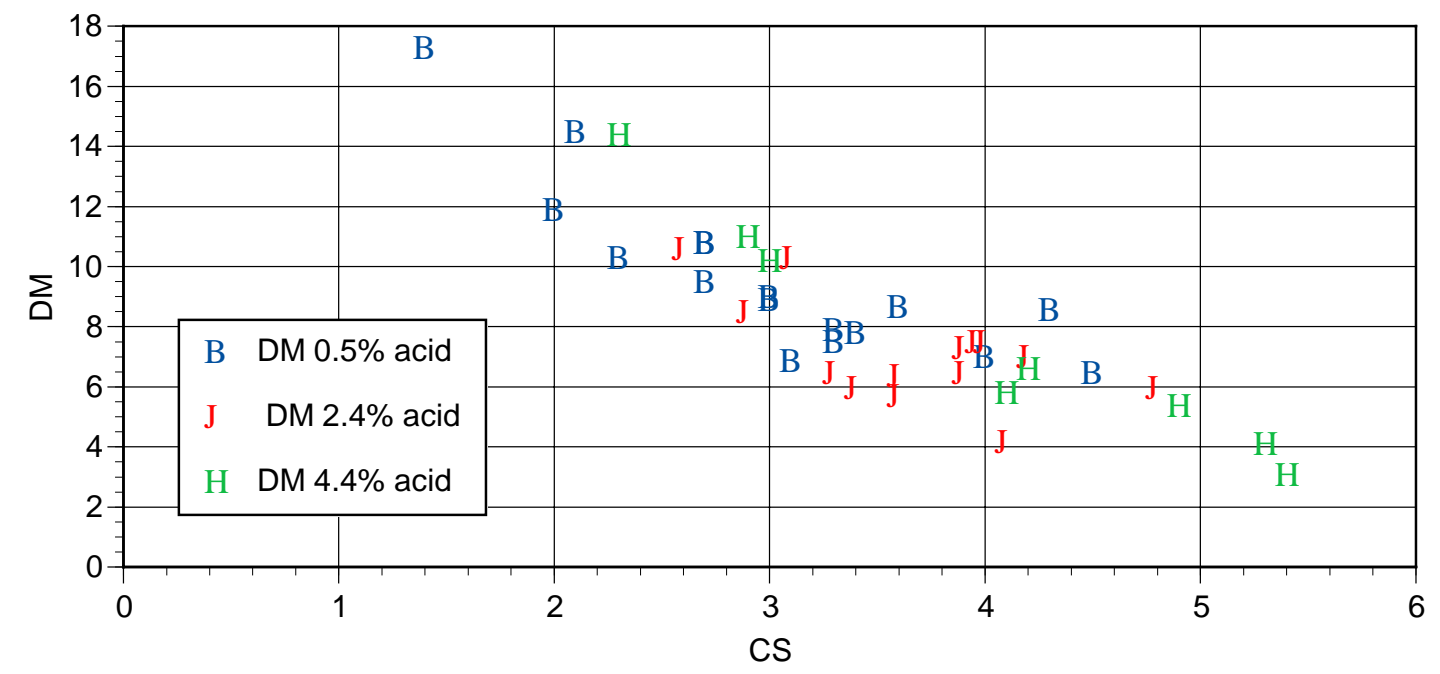

Figure 3.5 Residual dry matter versus $\log ($ Ro) for dilute sulfuric acid hydrolysis of pine wood [4]

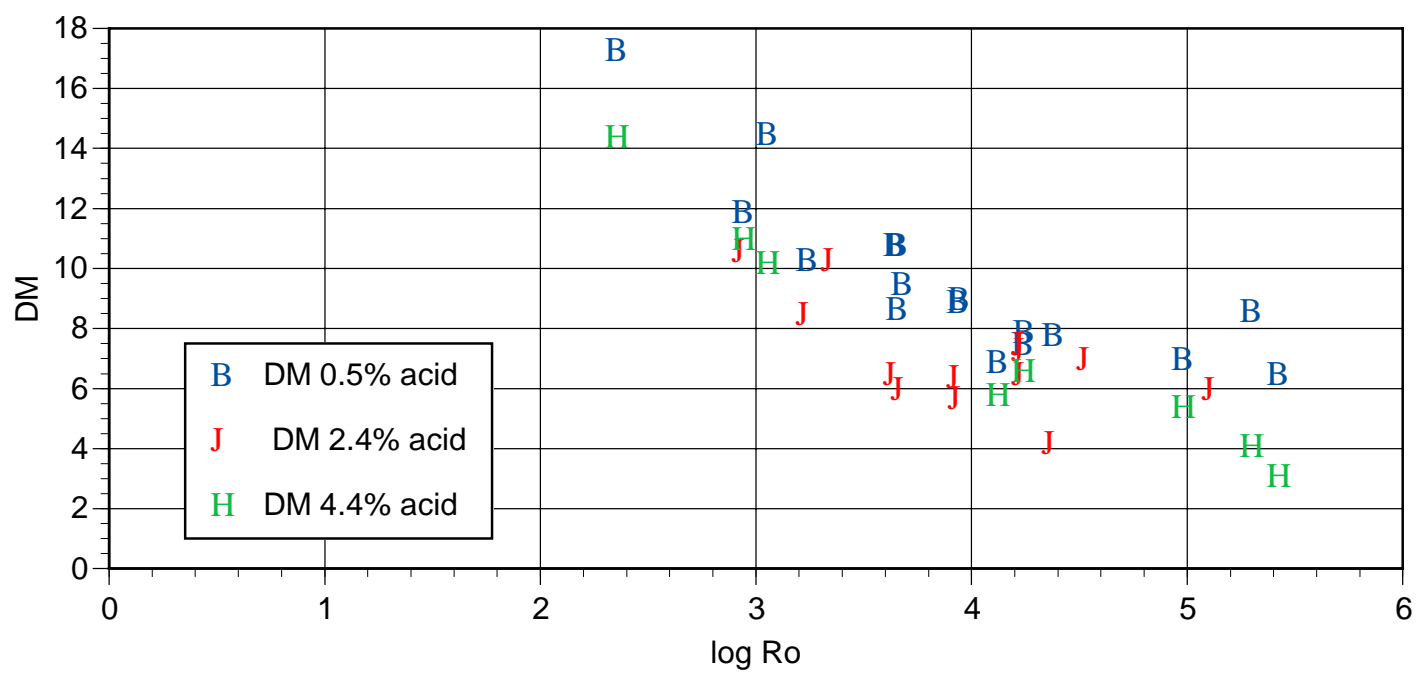




\section{Discussion + Conclusion}

From this brief investigation of recent and previously published results, it appears that the combined severity function, which is often used to characterize acid-catalyzed pretreatments, does indeed offer a better fit to solubilization data than does the regular severity factor when pretreatment is carried out at a $\mathrm{pH}$ lower than that resulting from the endogenous production of acids through thermal degradation. When added acids, such as carbonic acid, result in a $\mathrm{pH}$ similar to that of the endogenous acid production, use of the combined severity function appears to offer no improvement over the standard severity function.

\section{References:}

[1] McWilliams, Robert C. , van Walsum, G. Peter. 2002. Comparison of Aspen Wood Hydrolysates Produced by Pretreatment with Liquid Hot Water and Carbonic Acid. Accepted for publication in Applied Biochemistry and Biotechnology

[2] Overend, R. P., Chornet, E. 1987. Fractionation of lignocellulosics by steam-aqueous pretreatments. Phil. Trans. R. Soc. Lond A321, 523-536

[3] van Walsum, G. Peter. 2001. Severity Function Describing the Hydrolysis of Xylan using Carbonic Acid. Applied Biochemistry and Biotechnology. 91-93:317-329.

[4] Olsson, L. and Hahn-Hagerdal, B., Fermentation of lignocellulosic hydrolysates for ethanol production. Enyme Microb. Technol., 1996. 18: p. 312-331.

[5] Larsson, S., Palmqvist, E., Hahn-Hagerdal, B., Tengborg, C., et al., The generation of fermentation inhibitors during dilute acid hydrolysis of softwood. Enz. Microb. Technol., 1998. 24: p. 151-159. 


\title{
TASK 4 - Test the performance of a larger capacity reactor
}

\author{
Robert C. McWilliams, G. Peter van Walsum
}

\section{Summary}

Preliminary experiments using a $150 \mathrm{~mL}$ reactor were conducted using water and a range of $\mathrm{CO}_{2}$ pressures and reaction temperatures to evaluate the reactor performance and to determine when the pressure (and hence temperature) inside the reactor reaches steady-state. This revealed optimal reaction conditions and will minimize variation between experimental results generated from small $(15 \mathrm{~mL})$ and larger $(150 \mathrm{~mL})$ reactors. The data also provided an accurate determination of the time required for the reactor to reach reaction temperature.

\section{Introduction}

Up to this point, most experimental work with carbonic acid has been done on a small scale. This provided adequate volumes for studies of liquid hydrolysate composition, but does not provide sufficient volume of liquid or solid material to test hydrolysate inhibition or enzymatic hydrolysis rates of solids. Thus, this task served the purpose of testing the performance of a reactor to generate volume of samples for inhibition and enzymatic digestibility tests (task 5).

\section{Materials + Methods}

Apparatus and Materials:

$150 \mathrm{~mL}$ stainless steel reactor (Swagelok Corporation), sand bath (Techne, Oxford UK, model SBL 2D) and temperature controller (Techne model TC-8D); laboratory quality de-ionized water; and carbon dioxide from a pressurized cylinder.

Experimental design: Using a 10-fold scale-up from the $15 \mathrm{~mL}$ reactor, $80 \mathrm{~mL}$ of de-ionized water was placed in the $150 \mathrm{~mL}$ reactor. The reactor was then placed in a sand bath at temperatures of $180^{\circ} \mathrm{C}, 200^{\circ} \mathrm{C}, 220^{\circ} \mathrm{C}$, and $240^{\circ} \mathrm{C}$. At each temperature, initial $\mathrm{CO}_{2}$ pressures were 0 psi, $200 \mathrm{psi}, 400 \mathrm{psi}$, and $800 \mathrm{psi}$. While in the sand bath, internal reactor pressures were measured and recorded at the following time increments: $15 \mathrm{sec}, 30 \mathrm{sec}, 45$ sec, $1 \mathrm{~min}, 2 \mathrm{~min}, 3 \mathrm{~min}, 5 \mathrm{~min}, 10 \mathrm{~min}, 20 \mathrm{~min}, 30 \mathrm{~min}, 45 \mathrm{~min}$, and $1 \mathrm{hr}$. Pressures were evaluated to determine steady-state pressures/times at various temperatures and to correlate pressure/time intersections at different temperatures to yield a favorable pre-heat time to allow the larger reactor to be brought rapidly up to reaction temperature before placing the reactor in the sand bath at reaction temperatures.

\section{Results:}

Pressures measured at various times are recorded in table 4.1. Arrows in the table indicate the time required to reach the equilibrium pressure at $\mathrm{T}-40^{\circ} \mathrm{C}$. Figure 4.1 shows pressure/time curves for the reactor at 180 and $220^{\circ} \mathrm{C}$, charged with 800 psi of $\mathrm{CO}_{2}$. 
Pressure experiment results

\begin{tabular}{|c|c|c|c|c|c|c|c|c|c|c|c|c|}
\hline $\begin{array}{l}\text { Temp } \\
\text { (C) } \\
\end{array}$ & $\begin{array}{l}\mathrm{CO}_{2} \\
\text { pres } \\
\text { room } \\
\text { temp } \\
\text { (psig) } \\
\end{array}$ & $\begin{array}{l}\text { P at } \\
15 \mathrm{sec}\end{array}$ & $\begin{array}{l}\text { P at } \\
30 \text { sec }\end{array}$ & \begin{tabular}{|l|} 
P at \\
$45 s e c$
\end{tabular} & $\begin{array}{l}\text { P at } \\
\text { 60sec }\end{array}$ & \begin{tabular}{|l}
$P$ at \\
120 \\
sec
\end{tabular} & $\begin{array}{l}\mathbf{P} \text { at } \\
3 \mathrm{~min}\end{array}$ & $\begin{array}{l}P \text { at } \\
5 \mathrm{~min}\end{array}$ & $\begin{array}{l}P \text { at } \\
10 \mathrm{~min}\end{array}$ & $\begin{array}{l}P \text { at } \\
20 \mathrm{~min}\end{array}$ & $\begin{array}{l}P \text { at } \\
30 \mathrm{~min}\end{array}$ & $\begin{array}{l}P \text { at } \\
45 \mathrm{~min}\end{array}$ \\
\hline 180 & 0 & 0 & 0 & & 0 & 0 & 0 & 60 & 80 & 100 & 120 & 120 \\
\hline 180 & 200 & 200 & 220 & 235 & 250 & 260 & 280 & 305 & 360 & 400 & 410 & 420 \\
\hline 180 & 400 & & 480 & 500 & 520 & 580 & 620 & 670 & 690 & 690 & 690 & 690 \\
\hline 180 & 800 & 980 & 1060 & 1110 & 1170 & 1315 & 1420 & 1550 & 1680 & 1710 & 1720 & 1730 \\
\hline 200 & $0(1)$ & 80 & 120 & 135 & 150 & 200 & 240 & 300 & 350 & 380 & 380 & 380 \\
\hline 00 & $0(2)$ & 0 & 0 & 0 & 0 & 60 & 90 & 140 & 200 & 210 & 210 & 210 \\
\hline 200 & $0(3)$ & 0 & 0 & 0 & 0 & 60 & 90 & 150 & 200 & 210 & 210 & 210 \\
\hline 200 & 200 & 20 & 240 & 265 & 280 & 310 & 340 & 400 & 445 & 460 & 460 & 460 \\
\hline 200 & 400 & 50 & 500 & 520 & 550 & 620 & 665 & 715 & 760 & 780 & 780 & 780 \\
\hline 200 & $0(1)$ & 20 & & 30 & 1300 & 1520 & 1685 & 1880 & $2000+$ & 20004 & $2000+$ & $2000+$ \\
\hline 200 & $800(2)$ & 30 & 50 & 1130 & 1200 & 1380 & 1500 & 1640 & 1820 & 1900 & \begin{tabular}{|l|}
1920 \\
\end{tabular} & 1930 \\
\hline 200 & $800(3)$ & 980 & 1120 & 1260 & & 1540 & 1660 & 1800 & 1940 & 2000 & 2000 & 2000 \\
\hline 220 & $0(1)$ & 0 & 0 & 0 & 0 & 80 & 120 & 200 & 280 & 300 & 300 & 300 \\
\hline 220 & $0(2)$ & 0 & 0 & 0 & 0 & 70 & 115 & 190 & 280 & 310 & 320 & 320 \\
\hline 220 & & & 260 & 280 & 300 & 350 & 400 & 500 & & 800 & 600 & 600 \\
\hline 220 & 400 & 00 & 550 & 585 & 610 & 700 & 770 & 880 & 980 & 1000 & 1000 & 1000 \\
\hline 220 & $00(1)$ & & 1060 & 1150 & 1220 & 1400 & 1500 & 1640 & 1800 & 1820 & 1820 & 1820 \\
\hline 220 & $800(2)$ & 40 & 1200 & 1300 & 1420 & 1680 & 1820 & $2000+$ & $2000+$ & $2000+$ & $2000+$ & $2000+$ \\
\hline 220 & $800(3)$ & 1040 & 1200 & 1280 & 1380 & 1660 & 1840 & 20004 & $2000+$ & $2000+$ & $2000+$ & $2000+$ \\
\hline 240 & \begin{tabular}{|r|}
0 \\
\end{tabular} & 0 & 0 & 0 & 60 & 140 & 230 & 350 & 410 & 460 & \begin{tabular}{|l|}
460 \\
\end{tabular} & 460 \\
\hline 240 & $00(1)$ & & 160 & 260 & 1400 & 1600 & 1780 & 2000 & $2000+$ & $2000+$ & $2000+$ & $2000+$ \\
\hline 240 & $800(2)$ & 1040 & 1200 & 1320 & 1420 & 1760 & 1930 & $2000+$ & $2000+$ & $2000+$ & $2000+$ & $2000+$ \\
\hline
\end{tabular}


Figure 4.1. Determination of reactor heat up time at reaction temperature $+40^{\circ} \mathrm{C}$

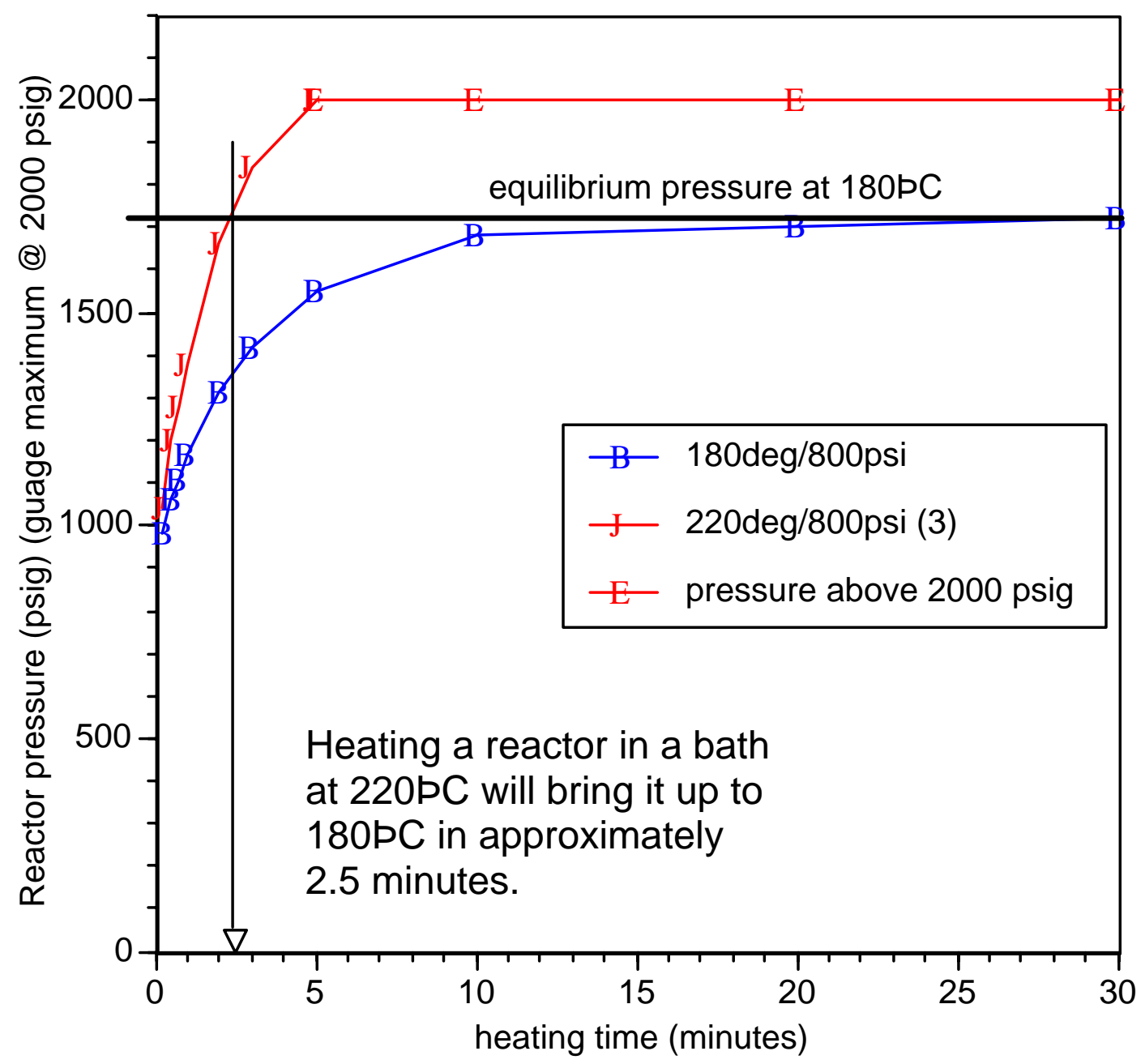

Discussion: At 0 psi for all temperatures, the larger reactor had a slower initial heat-up time compared with experiments conducted under the same conditions with a $15 \mathrm{ml}$ stainless steel reactor of similar design. Given the larger size of the reactor, this was expected. Typically, at this initial pressure it took 2 minutes for the pressure to begin to increase gradually followed by a more pronounced pressure increase and finally a "leveling-off" as the reactor's internal pressure reached steady-state. At 200 psi, 400 psi, and 800 psi, initial pressures, there was a gradual initial increase, followed by a more rapid increase between 3 and 5 minutes, then returning to a gradual increase and leveling-off between 5 and 45 minutes. This experiment provided an excellent data set to determine pressure/time intersections of reactions at different temperatures. From this data set it was determined that a 40 degree temperature increase (keeping initial pressure the same) results in reaching reaction pressure of the target reaction temperature in considerably less time. For example, a reaction at $180^{\circ} \mathrm{C}$ and 800 psi initial $\mathrm{CO}_{2}$ pressure took 30 minutes to reach a steady-state pressure of $1720 \mathrm{psi}$. In contrast, a reaction at $220^{\circ} \mathrm{C}$ and 800 psi took between 2 and 3 minutes to reach the same pressure. Therefore, when conducting reactions at $180^{\circ} \mathrm{C}$, preheating the reactor (with the water or water $/ \mathrm{CO}_{2}$, and wood already inside) at $220^{\circ} \mathrm{C}$ would allow the reactor to reach steady state much faster and yield more consistent results. 
Conclusion: The $150 \mathrm{~mL}$ reactor design is adequate in design to allow the scale up of previous experiments and to produce a larger volume of product for further evaluation. Results from experiments with water alone were comparable to published steam table data at each temperature [1] and consistent with results for the $15 \mathrm{~mL}$ reactor under the same circumstances. The determination of an accurate preheat time and temperature allows the reactor to begin the reaction at steady-state temperature which will provide more consistency and a truer picture of the reaction a the established reaction time.

\section{References:}

[1] Chemical Rubber Company, Handbook of Chemistry and Physics, $62^{\text {nd }}$ Edition, CRC press, Boca Raton, FL, 1982-2, E-17, 18 


\title{
TASK 5 - Generate larger samples at selected reaction conditions
}

\author{
Robert C. McWilliams, G. Peter van Walsum
}

\section{Summary}

The newly constructed $150 \mathrm{~mL}$ reactor was used in reactions that replicated the conditions of the $15 \mathrm{~mL}$ reactor. 1.0 grams of aspen wood, $80 \mathrm{ml}$ of de-ionized water were reacted with and without $\mathrm{CO}_{2}$ at 800 psig. Reaction temperatures were $180^{\circ} \mathrm{C}, 200^{\circ} \mathrm{C}$, and $220^{\circ} \mathrm{C}$ with reactions times of 8,16 , and 32 minutes. The reactor was preheated in a sand bath set to a temperature $40^{\circ} \mathrm{C}$ above reaction temperature for two minutes. This allowed the reactor to quickly reach reaction temperature, as determined and reported in the previous progress report. The $150 \mathrm{ml}$ reactor successfully delivered the expected 10 -fold increase in hydrolysate compared to the $15 \mathrm{ml}$ reactor. Results of the $\mathrm{pH}$ and $\mathrm{UV}$ analysis of the hydrolysate were consistent with those yielded by the $15 \mathrm{ml}$ reactor.

\section{Introduction}

Up to this point, most experimental work with carbonic acid has been done on a small scale. This provided adequate volumes for studies of liquid hydrolysate composition, but does not provide sufficient volume of liquid or solid material to test hydrolysate inhibition or enzymatic hydrolysis rates of solids. This task served the purpose of testing generating volume of samples for inhibition and enzymatic digestibility tests (tasks 6 and 7).

\section{Materials + Methods}

Apparatus and Materials: $150 \mathrm{~mL}$ stainless steel reactor (parts supplied by Swagelok Corporation); two sand baths (Techne, Oxford UK, model SBL 2D) and temperature controllers (Techne model TC-8D); laboratory quality de-ionized water; and carbon dioxide from a pressurized cylinder, ground Aspen wood using a domestic coffee grinder, stainless steel sieve (2 mm to 500 micron mesh), pH meter (Acumet AR-15, Fisher Scientific), spectrophotometer (Beckman, DU-600), centrifuge (Eppendorf) laboratory balance (Ohaus, Explorer)

Experimental: Aspen wood chips are ground using a domestic coffee grinder and sifted in the stainless steel sieve. The wood particles are allowed to separate and the particles between 0.5 $\mathrm{mm}$ and $1 \mathrm{~mm}$ are retained for experimental use. 1 gram samples of the $0.5-1 \mathrm{~mm}$ wood particles are weighed and placed in the reactor. In addition to the wood, $80 \mathrm{~mL}$ of water is added. The reaction temperatures $\left(180,200,220^{\circ} \mathrm{C}\right)$ and times $(8,16,32$ minutes) selected are based on previous results using the smaller reactor. At each of these sets of conditions, reactions were run using wood and water alone (as previously described) and wood, water, and $\mathrm{CO}_{2}$ at $800 \mathrm{psi}$. The reactor was placed in one sand bath set at a temperature 40 degrees Celsius higher than the desired reaction temperature for three minutes. This allowed the reactor to reach steady-state before immersion into the sand bath at reaction temperature. The $\mathrm{pH}$ of the hydrolysate was measured. Finally, spectrophotometric measurements of the hydrolysate (1\% solutions) were made using the spectrophotometer in scan wavelength mode between $190 \mathrm{~nm}$ and $350 \mathrm{~nm}$. Absorption peaks along this spectrum were compared in reactions with and without $\mathrm{CO}_{2}$ to evaluate the concentration of degradation products that are potentially inhibitory to enzymatic digestion. 


\section{Results:}

Table 5.1 and figures 5.1 and 5.2 illustrate data generated to date using the larger volume reactor.

Table 5.1

\begin{tabular}{|l|r|r|r|}
\hline temp & time & $\mathrm{pH}$ wood & $\mathrm{pH} \mathrm{CO2}$ \\
\hline $200 \mathrm{C}$ & 8 & 3.81 & 3.76 \\
\hline $200 \mathrm{C}$ & 16 & 3.51 & 3.96 \\
\hline $200 \mathrm{C}$ & 32 & 3.44 & 3.58 \\
\hline $220 \mathrm{C}$ & 8 & 3.68 & 3.42 \\
\hline $220 \mathrm{C}$ & 16 & 3.34 & 3.34 \\
\hline $220 \mathrm{C}$ & 32 & 3.44 & 3.26 \\
\hline
\end{tabular}

Figure 5.1

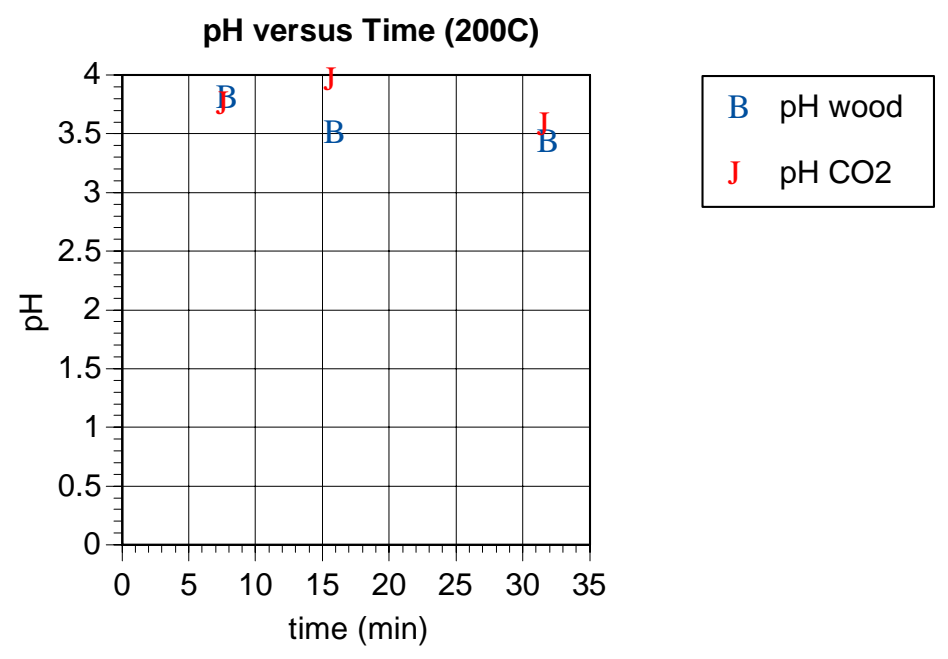

Figure 5.2

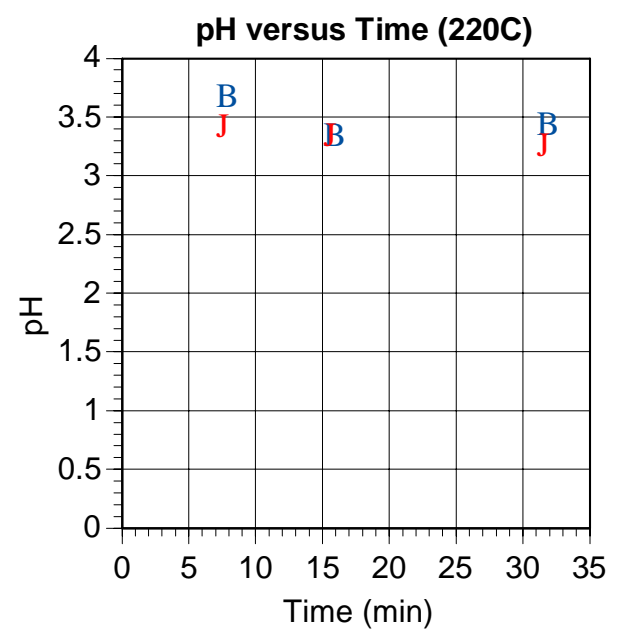

$\begin{array}{ll}\mathrm{B} & \mathrm{pH} \mathrm{CO} 2 \\ \mathrm{~J} & \mathrm{pH} \text { Water }\end{array}$ 
Discussion and Conclusion: The 150ml reactor met all performance expectations generating larger volumes of hydrolysate which was consistent with hydrolysate yielded by the smaller reactor. This larger volume of hydrolysate will enable faster analysis of toxicity and an applied understanding of the composition of the hydrolysate. 


\title{
Task 6: In vitro determination of inhibition
}

\author{
Damon Yourchisin, G. Peter van Walsum
}

\section{Summary}

Inhibition tests measured the rate of sugar consumption by Saccharomyces cerevisiae growing in batch culture of hydrolysate. It was found that inhibition of the yeast culture increased with severity of pretreatment above a mid level severity. Below this severity, little to no inhibition was observed. No difference was observed between the inhibition of hydrolysates prepared either with or without the presence of $\mathrm{CO}_{2}$. To conduct the experiments, serum vials were charged with $21 \mathrm{~g} / \mathrm{L}$ of sterile growth medium containing $20 \mathrm{ml}$ of pretreatment hydrolysate. The vials were inoculated with $0.2 \mathrm{ml}$ of freshly grown cell broth and incubated. Glucose concentrations over time were determined via glucose assay (Infinity Glucose Reagent) and the HPAE when available.

\section{Introduction}

The organisms used for the fermentation of ethanol are often inhibited by the degradation products produced during pretreatment. Enzymatic hydrolysis can also be impaired by inhibitors produced during pretreatment. Inhibitory compounds originate from: the hydrolysis of extractive components, organic and sugar acids (e.g. acetic, formic, glucuronic, galacturonic) esterified to hemicellulose; solubilized phenolic lignin derivatives; the degradation of solubilized compounds (e.g. furfural, hydroxymethyl furfural); and the release of corrosion products (e.g. metal ions)]. The production of inhibitors has been documented for dilute-acid, steam-explosion, acid-hydrolysis and liquid hot water pretreatments. To date no published studies have looked at inhibition resulting from carbonic acid pretreatment. Treatments to eliminate or reduce inhibitors, such as ion exchange and over-liming, add considerable expense to processing systems.

\section{Materials + Methods}

\section{Apparatus and materials}

Two Techne Fluidised Sand Baths, model SBL-2d, with temperature controllers.

Beckman DU 520 General Purpose UV/Vis Spectrophotometer (with single cell module)

Fisher Scientific AR15 pH meter by Accumet Research

$150 \mathrm{ml}$ stainless steel immersible reactors

Eppendorf Reference series auto pipettes

Ohaus Expolorer digital scale Item \#12140 (d=0.1mg)

Domestic brand coffee grinders

Fisher Scientific U.S.A. Standard Testing Sieve, ASTME-11 Spec \#18 (1mm opening)

Standard laboratory de-ionized quality water

Standard laboratory grade carbon dioxide

Vacuum filter with water aspirator

Schleicher and Schuell Sharkskin 100Cir. 5.5cm Microfilter paper

Sigma Yeast Malt Agar@ 41g/L

Sigma Yeast Malt Broth @ 21g/L

Fleischmann's Active Dry Yeast

Fisher Scientific Marathon 21000R centrifuge

Market Forge Sterilmatic STM-E autoclave 
Thermolyne MaximixII type 37600 mixer

SIGMA Diagnostics - Infinity ${ }^{\mathrm{TM}}$ Glucose Reagent (Procedure 17-UV) Kit

Glass serum vials $(50 \mathrm{~mL})$

Aspen wood chips (provided by USDA Forest Products Laboratory in Madison, WI)

Sigma D-Glucose Anhydrous

\section{Experimental Methods}

The experiments followed a general methodology regardless of reaction conditions. The general steps included: grinding the feedstock, pretreatment, preparation of hydrolysate, separating the solids from the liquids, microbial culturing, inhibition tests, analysis of the samples. These steps are detailed below and summarized in figure 6.1.

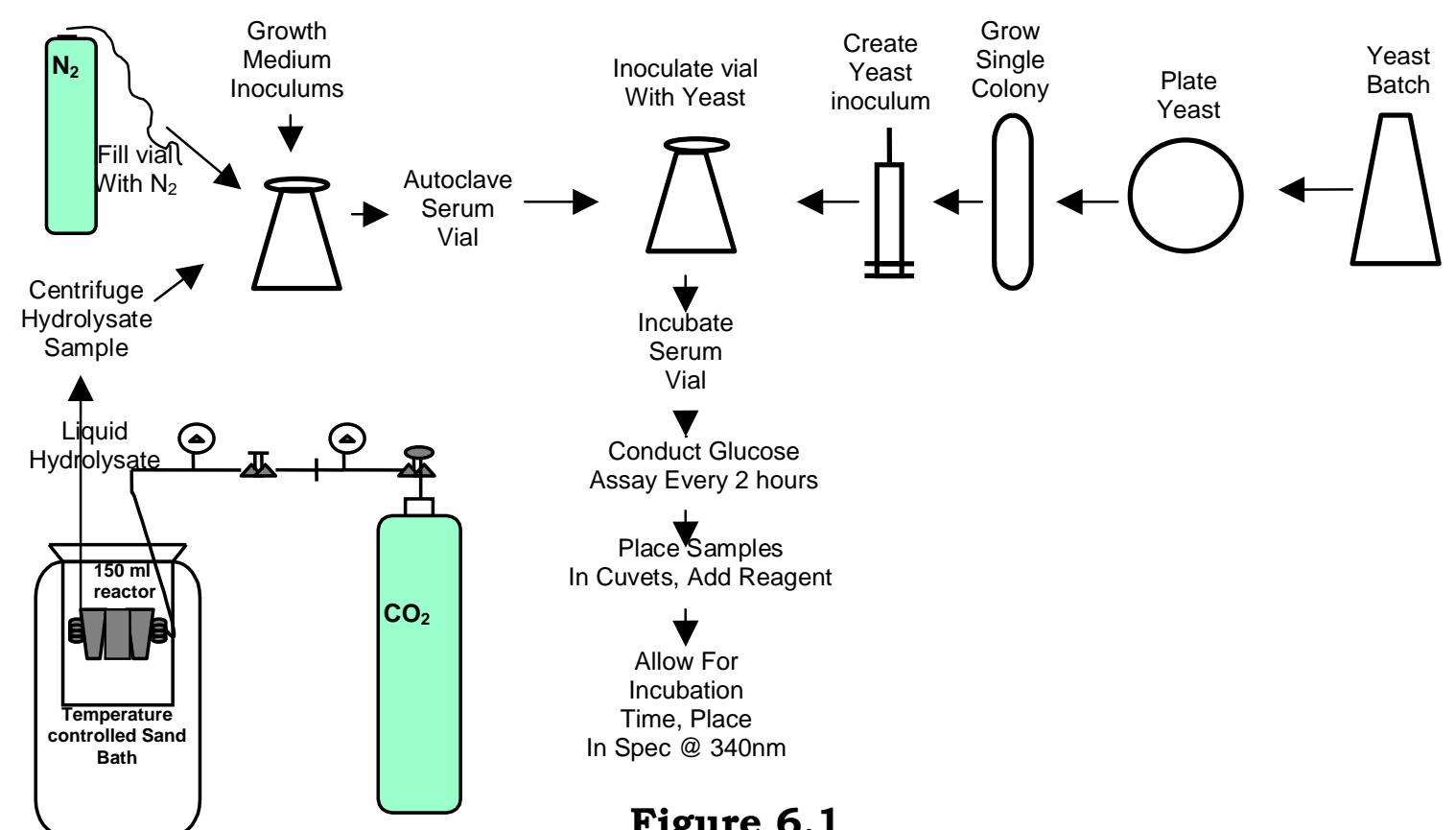

\section{Feedstock Preparation}

Feedstock was ground and sifted. Particles that passed through a $1 \mathrm{~mm}$ screen were used, particle sizes of $0.6-1 \mathrm{~mm}$. Two samples of $1.25 \mathrm{~g}$ each of uniform particulate sized feedstock were weighed out and placed in separate $150 \mathrm{ml} 316$ stainless steel reactors, along with $100 \mathrm{ml}$ of DI water each. The reactors were filled and emptied by removing a swage connection on one end. One reactor was pressurized with $800 \mathrm{psi}$ of $\mathrm{CO}_{2}$. For reactions using $\mathrm{CO}_{2}$, a 1/8” and1/4” stainless steel tubing connection and valve with pressure gage were fitted to the reactor.

\section{Pretreatment}

One sand bath was heated to the desired reaction temperature. The other sand bath was heated to $40^{\circ} \mathrm{C}$ above the desired reaction temperature. The higher temperature sand bath was used for preheating the reaction vessels to better control thermal and pressure equilibrium. A 3 minute pre-heat at reaction temperature plus $40^{\circ} \mathrm{C}$ allowed the reactors to quickly reach a state of constant pressure, thereby reducing the variability of reaction dynamics when compared to a slower heat-up. (27) Both filled reactors were placed into the reaction temperature plus $40^{\circ} \mathrm{C}$ sand bath for 3 minutes as a preheat. They were immediately removed and placed in the reaction temperature sand bath for the desired reaction duration. 
The reaction was quenched in an ice bath immediately after the reaction duration time period was complete.

Reaction times consisted of short durations (4-8 minutes), a mid-range duration (16 minutes), and long-range durations (32-64 minutes). Pretreated samples were extracted from the reactors and placed in glass collection bottles for the next step.

\section{Preparation of hydrolysates}

Solids were filtered out from the hydrolysate samples generated using vacuum filter and microfilter paper. The solids remaining on the filter paper were washed with DI water 3 times (12ml DI water total). During the final rinse the vacuum filter was allowed to work for 3 minutes and then shut off to collect solids. The solids were placed in weighing tins in preparation for enzymatic hydrolysis experiments. The liquids were then centrifuged to remove all solids. The original liquid was placed into centrifuge bottles and centrifuged at 4000rpm for 15 minutes at $15^{\circ} \mathrm{C}$. The centrifuge bottles were emptied using a pipette, to ensure no solids were present, into clean collection bottles. The liquid hydrolysate was now prepared for experimentation.

\section{Microbial culturing}

A new batch of yeast agar, yeast broth, and yeast were prepared for each experiment according the manufacturers directions. Yeast agar and broth were prepared in the following manner: $500 \mathrm{ml}$ of water were brought to a boil and 20.5grams of yeast agar or 10.5grams of yeast broth were placed into it. Once all solids were dissolved, the mixtures were autoclaved. After autoclaving, $20 \mathrm{ml}$ of the agar were aseptically transferred from the autoclaved flask to a Petri dish and $10 \mathrm{ml}$ of the broth were aseptically transferred from the autoclaved flask to a culture tube under the laminar flow hood. Three plates and three culture tubes were created each time to ensure growth and to act as a back-up during each step if contamination occurred. A new batch of bakers yeast was prepared according to manufacturers directions: 2 $1 / 4$ teaspoons of yeast was added to $1 / 4$ cup of water at 1000F with 1 teaspoon of glucose. The mixture was let to stand for 10 minutes. Once growth of yeast was confirmed, they were plated for isolation under the laminar flow hood using the fresh yeast agar plates. After plating, the Petri dishes were placed in the incubator for 48 hours at $30^{\circ} \mathrm{C}$ along with the culture tubes of broth (although nothing was in them yet). After 48 hours, the plates and broth culture tubes were removed and put under laminar flow hood in order to aseptically transfer a single yeast colony from the agar plate to the broth culture tube. An isolated colony from the plate was aseptically transferred into the $10 \mathrm{ml}$ of broth in the culture tube using a loop. The plates were properly disposed of and the culture tubes were placed into the incubator at $30^{\circ} \mathrm{C}$ for 48 hours.

\section{Preparation of test vials}

Twenty milliliters of the previously generated liquid hydrolysate were placed into serum vials. $20 \mathrm{ml}$ was the maximum amount of liquid per vial. The vials contained from $0 \%$ to $100 \%$ hydrolysate. The remaining $20 \mathrm{ml}$ of the less than $100 \%$ hydrolysate was made up using DI water. Yeast broth at $21 \mathrm{~g} / \mathrm{L}(0.42 \mathrm{~g})$ was weighed and placed into each serum vial to serve as a known growth medium. Prior to autoclaving the serum vials $\mathrm{N}_{2}$ gas was bubbled in them for 30 seconds. Each vial was capped immediately and sealed after removal from the $\mathrm{N}_{2}$ injector. Once all vials were sealed, they were placed in the autoclave for 20 minutes at $121^{\circ} \mathrm{C}$. The $\mathrm{pH}$ for each of these samples was tested and found to be within the tolerance range of yeast, as shown in table 6.1 below for the most recently performed experiment.

Table 6.1 pH of hydrolysates prior to yeast cultivation 


\begin{tabular}{|l|c|}
\hline \multicolumn{2}{|c|}{$\begin{array}{c}\text { Exp 138:pH of } \\
\text { hydrol+nutrient }\end{array}$} \\
\cline { 1 - 2 } $\begin{array}{c}\text { Liquid } \\
\text { Samp }\end{array}$ & \multirow{2}{*}{$\mathrm{pH}$} \\
\cline { 1 - 2 } $\mathrm{T} / \mathrm{t} / \mathrm{P}$ & \\
\hline $180 / 8 / 0$ & 5.69 \\
\hline $180 / 8 / 800$ & 5.87 \\
\hline $200 / 16 / 0$ & 5.04 \\
\hline $200 / 16 / 800$ & 5.05 \\
\hline $220 / 4 / 0$ & 5.25 \\
\hline $220 / 4 / 800$ & 5.39 \\
\hline $180 / 64 / 0$ & 5.01 \\
\hline $180 / 64 / 800$ & 4.97 \\
\hline $0 \%$ hydrol & 6.31 \\
\hline
\end{tabular}

\section{Inhibition test}

Once the vials were cooled they were placed in the incubator to bring them to the same temperature as the yeast broth that would be transferred to them. Once the yeast broth had been incubated for exactly 48 hours, the serum vials were each injected $0.2 \mathrm{ml}$ of the vortexed yeast inoculum from the culture tubes using a $1 \mathrm{ml}$ syringe. This was performed under the laminar flow hood and each serum vial top was flamed prior to needle insertion. This became time zero $(\mathrm{T}=0)$ for the experiment. Samples were taken for the next 12 hours every 2 hours, except for the first 4 hours, $T+4$ was the second measurement.

\section{Glucose Assay}

At each testing period, $0.2 \mathrm{ml}$ of sample was removed from the serum vials and place into a $1 \mathrm{ml}$ microcentrifuge tube. The sample was diluted $5: 1$, so $0.8 \mathrm{ml}$ of DI water was added to each centrifuge tube. This was done because the yeast broth contained $10 \mathrm{~g} / \mathrm{l}$ dextrose and the glucose assay was only good for up to $5 \mathrm{~g} / \mathrm{l}$, but $2 \mathrm{~g} / \mathrm{l}$ was best. Next, 1500 microliters of glucose reagent was placed into a $2 \mathrm{ml}$ cuvette for each sample, minimizing the time the glucose reagent is out of the refrigerator as much as possible. Using the spectrophotometer, UV measurements of the reagent only were taken and recorded. This was done by placing each cuvette in the spectrophotometer set at 340nm absorbance and measuring the AUs. Then, 15 microliters of each sample were removed and placed in their respective $2 \mathrm{ml}$ cuvettes. The cuvettes were covered and incubated for 14 minutes (ambient room temperature should be between $20^{\circ} \mathrm{C}$ and $22^{\circ} \mathrm{C}$ ). Serum vials were immediately placed in the incubator at $30^{\circ} \mathrm{C}$. After 14 minutes, each cuvette was again placed in the spectrophotometer set at 340nm absorbance and was measured and recorded. The recorded AUs of each cuvette with reagent only were subtracted from the recorded AUs of the cuvettes with sample and reacted reagent. A glucose standard of $2 \mathrm{~g} / \mathrm{l}$ was run at each sampling time in order to compare the samples to a known standard to determine glucose generated throughout the experiment. At $\mathrm{T}+24$ the dilution was only 1:1 for the samples and at $\mathrm{T}+48$ there was no dilution of sample prior to analysis with the assay. The glucose assay was found to be linear up to $3 \mathrm{~g} / \mathrm{L}$ of glucose.

This information was used when calculating theoretical glucose yield of samples prior to experimentation to ensure they would be within the range of the assay's testing limits. It was also used to determine the correct $\mathrm{g} / \mathrm{L}$ of glucose standard to run as a standard for each experiment, which was chosen to be a constant $2 \mathrm{~g} / \mathrm{L}$ for each experiment. A control was also 
used for each yeast inhibition experiment by running a sample that contained $0 \%$ hydrolysate but all the other components of the nutrient broth.

\section{Results}

Data were analyzed using the DU 500 Spectrophotometer, $\mathrm{pH}$ meter and Infinity Glucose Reagent Assay kit. Two metrics were used, total recovered glucose and reaction speed. The raw numbers were averaged amongst duplicates within experiments and graphed (Absorbance Units versus time, transposed into Glucose yield versus time).

Figure 6.2 shows that there is no effective difference in the yeast growth rates in hydrolysates produced either with or without the presence of $\mathrm{CO} 2$. These experiments were carried out at the mid point severity of the study, at $200 \mathrm{C}$ and 16 minutes reaction time.

Figure 6.2

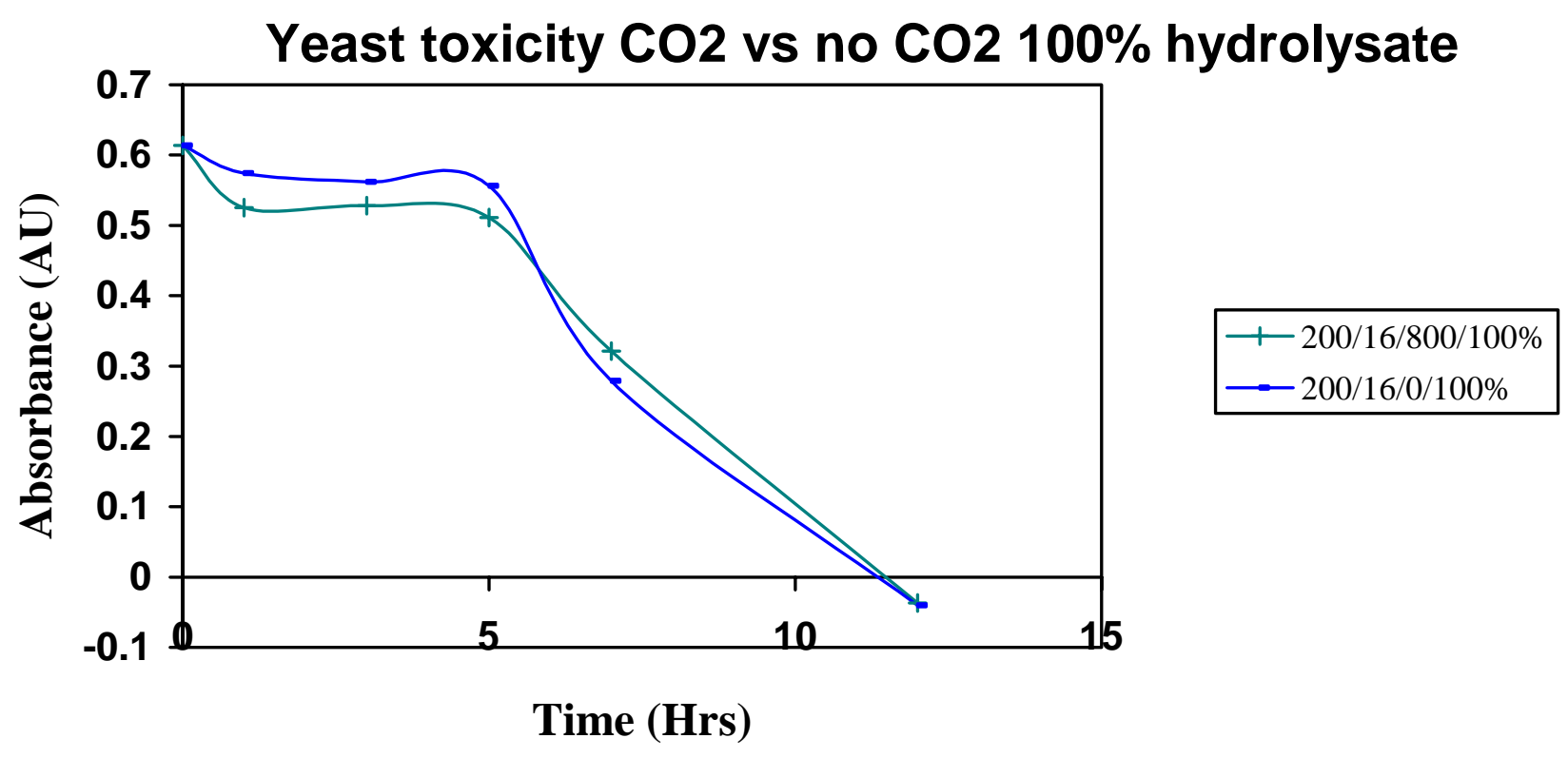


Figure 6.3 below, again shows that there is no difference between samples that contain $\mathrm{CO}_{2}$ and samples without $\mathrm{CO}_{2}$. In this case, a marked difference is evident in the inhibition of the yeast by hydrolysates produced at high ( 220 C, 32 minutes) and low (180 C, 8 minutes) severities.

Figure 6.3

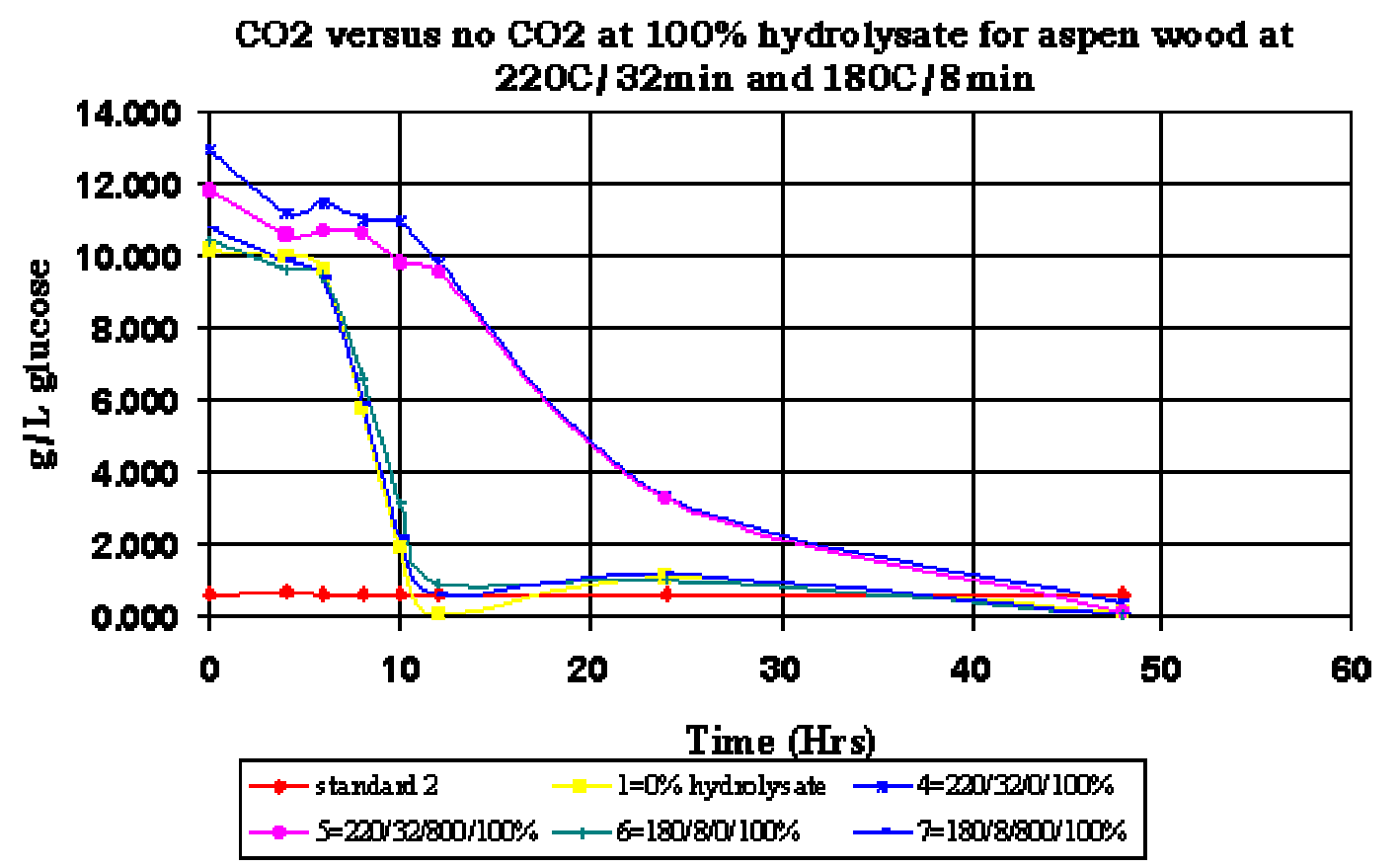

At the lowest tested severity parameter of $180^{\circ} \mathrm{C}$ for 8 minutes, there was almost no inhibition when compared to the control that was run at each experiment. However, at the highest tested severity parameter of $220^{\circ} \mathrm{C}$ for 32 minutes, there was an almost $50 \%$ reduction in the speed of glucose consumption by the yeast. This division of inhibition as it correlates to severity is well repeated among the samples. 
Figure 6.4 illustrates the effect of dilution of the inhibition by the hydrolysates. It can be seen that diluted hydrolysates are less inhibitory, thus there appears to be a relatively continuous dose-response to the inhibitors.

Figure 6.4

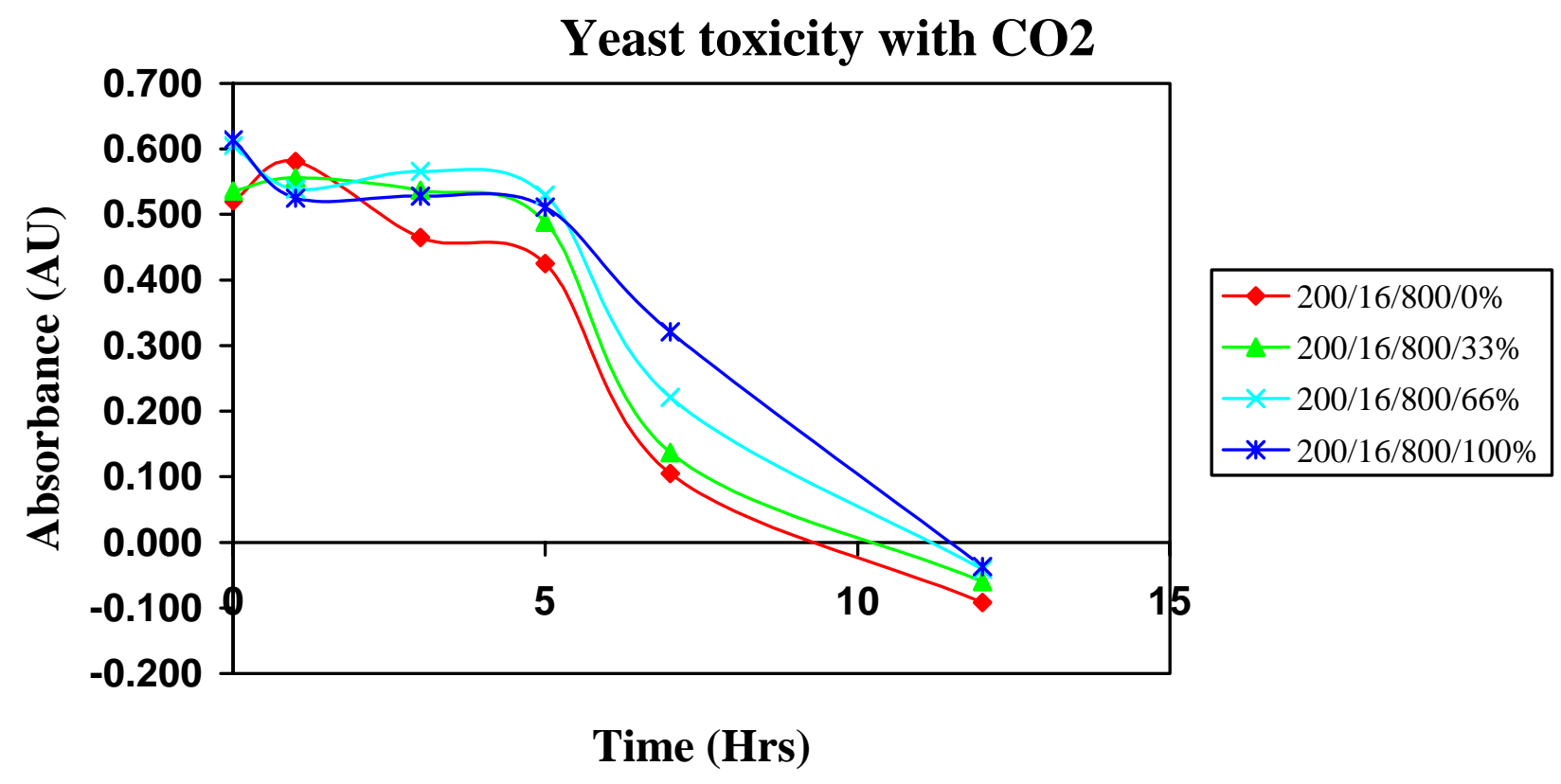

\section{Discussion}

The midpoint pretreatment condition of $200^{\circ} \mathrm{C}$ for 16 minutes could be an optimal condition for reduced microbial inhibition. For severities around the midpoint and below, there was no improvement in the reduction of microbial inhibition. A possible explanation for this result is that at the midpoint and below conditions, there are not enough inhibitory compounds released to effect the hearty yeast organisms. However, the more severe reaction conditions, above the midpoint, are continuing to breakdown the biomass and releasing more and more inhibitory compounds into the hydrolysate, thereby inhibiting the yeast by making their environment more difficult to survive in, but not impossible and not killing them.

\section{Conclusion}

Pretreatment with carbonic acid showed no significant advantage when compared to pretreatment with liquid hot water. Differences between the samples could be attributed to testing error since in some cases the samples with carbonic acid showed reduced inhibition rates and yields and in other cases they showed an increase in inhibition rates and yields. 


\title{
Task 7: Determine enzyme digestibility of pretreated solids
}

\author{
Damon M. Yourchisin, G. Peter van Walsum
}

\begin{abstract}
Summary
Enzyme digestibility tests measured enzymatic hydrolysis rates of pretreated solids by cellulase enzymes (Novozyme 188 and Iogen cellulase). It was found that more severe pretreatments enhanced enzymatic digestibility. The addition of pressurized $\mathrm{CO}_{2}$ to the pretreatment system did not significantly increase enzymatic hydrolysis rates compared to water-alone pretreatment. To conduct the experiments, $\mathrm{s}$ erum vials were charged with a $\mathrm{pH}$ 5.0 buffer, preservative, enzyme and pretreated solid sample estimated to have $2 \mathrm{~g} / \mathrm{L}$ cellulose (calculated from dry weight of the solid residue) and incubated in a $40^{\circ} \mathrm{C}$ shaker bath. Glucose concentrations over time were determined via glucose assay and the HPAE when available.
\end{abstract}

\section{Introduction}

The main purpose of pretreatment is to enhance fiber reactivity to enzymatic hydrolysis. The rate of enzymatic digestion is important because higher rates increase productivity and allows for use of less enzyme. Increased fiber reactivity is accomplished through a variety of mechanisms: solubilization of hemicellulose , removal of lignin , reduction of particle size, alteration of the cellulose characteristics such as degree of polymerization, abundance of cellulose chain ends and crystallinity. Effective pretreatments in general approach or exceed $80 \%$ of theoretical cellulose conversion upon subsequent hydrolysis of a representative hardwood feedstock (e.g. poplar) using moderate (e.g. 10 to $15 \mathrm{FPU} / \mathrm{g}$ cellulose) cellulase loadings. Such conversions are achieved in a period on the order of five days, although this is highly feedstock-dependent.

\section{Materials + Methods}

$\underline{\text { Apparatus and materials }}$

Two Techne Fluidised Sand Baths SBL-2d with temperature controllers.

Beckman DU 520 General Purpose UV/Vis Spectrophotometer (with single cell module) $150 \mathrm{ml}$ stainless steel imersible reactor

Ohaus Expolorer digital scale Item \#12140 (d=0.1mg)

Cole Parmer 2ml disposable plastic cuvettes

Domestic brand coffee grinders

Fisher Scientific U.S.A. Standard Testing Sieve, ASTME-11 Spec \#18 (1mm opening)

Standard laboratory de-ionized quality water

Standard laboratory grade carbon dioxide

Vacuum filter with water aspirator

Schleicher and Schuell Sharkskin 100Cir. 5.5cm Microfilter paper

Blue $\mathrm{M}$ drying oven model OV-18A set at $100^{\circ} \mathrm{C}$ and $40^{\circ} \mathrm{C}$

New Brunswick Scientific Reciprocal Water Bath model R76

Thermolyne MaximixII type 37600 mixer

SIGMA Diagnostics - Infinity ${ }^{\mathrm{TM}}$ Glucose Reagent (Procedure 17-UV) Kit

Glass serum vials $(50 \mathrm{~mL})$ and $(100 \mathrm{~mL})$

Novo Nordisk's cellobiase (Novozyme 188L cellulase enzyme)

Iogen cellobiohydrolase, endoglucanase, and B-glucosidase (Iogen cellulase enzyme) 
Citric Acid, 99\%

Benzoic Acid, 99\%

Sulfuric Acid, $72 \%$

Sodium Hydroxide Solution, 50/50 w/w

Aspen wood chips (provided by USDA Forest Products Laboratory in Madison, WI)

Aspen wood liquid hydrolysate

Avicel pure dried cellulose

Sigma D-Glucose Anhydrous

\section{Experimental Design}

Enzyme digestibility of pretreated solids at varying parameters around the midpoint of $\left(200^{\circ} \mathrm{C} / 16 \mathrm{~min} /\right.$ with and without $\left.\mathrm{CO}_{2}\right)$ for both aspen wood and corn stover was measured. An assessment of hydrolysis enhancement of pretreatment with carbonic acid $\left(\mathrm{CO}_{2}\right)$ was then made. The general steps included: preparation of the feedstock, pretreatment, separating the solids from the liquids, drying some of the solids and calculating dry weight; preparation of the testing vials, enzymatic hydrolysis tests, performing a glucose assay on the samples. The following procedure is summarized in figure 7.1, below.

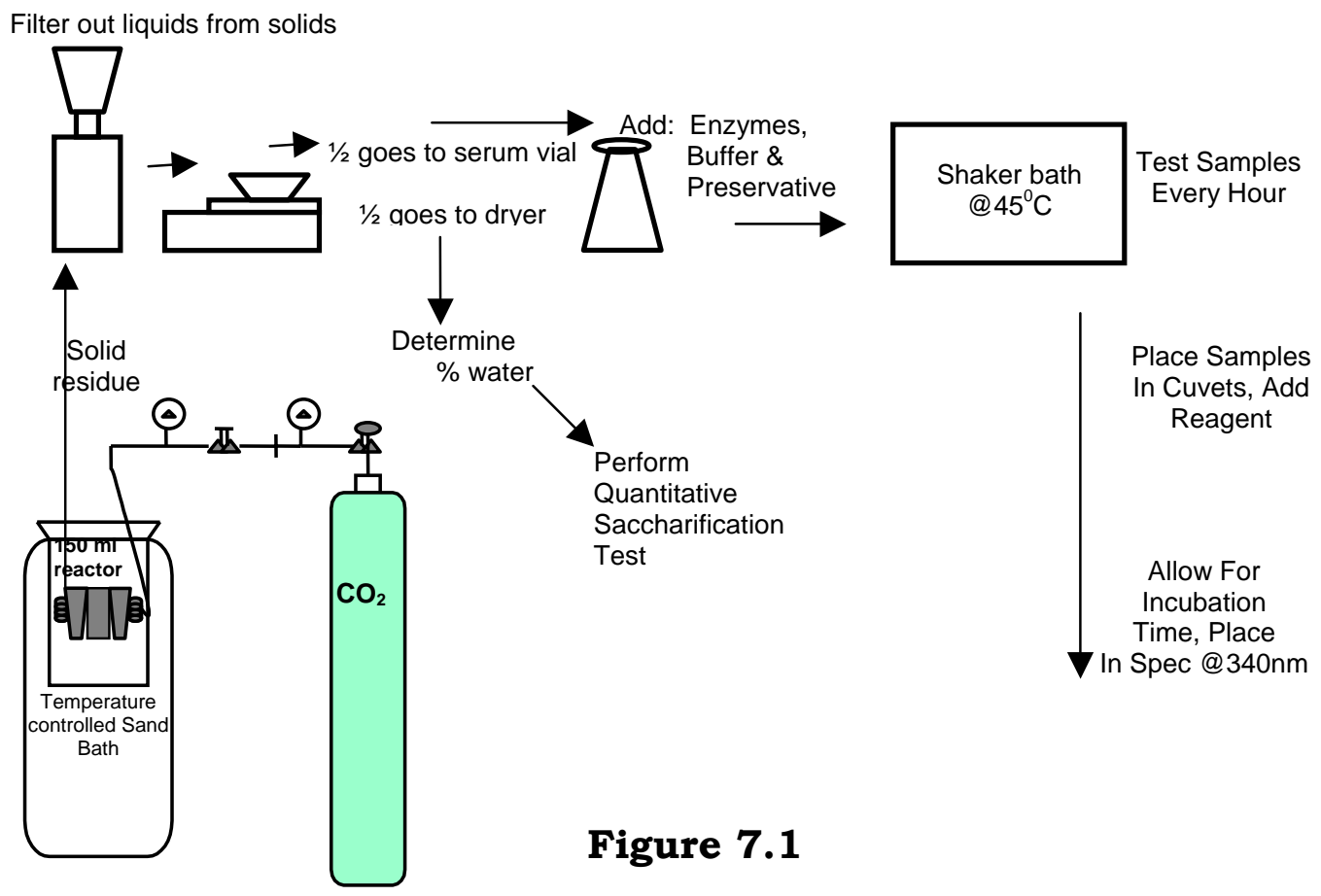

\section{Preparation of feedstock}

This step used the same methods as detailed under task 6 .

\section{Pretreatment}

This step used the same methods as detailed under task 6.

Preparation of the solid residue 
The solids were separated from the combined reactor contents using a vacuum filter and microfilter paper. The solids remaining on the filter paper were washed with DI water 3 times (12ml DI water total). During the final rinse the vacuum filter was allowed to work for 3 minutes and then shut off to collect solids. The solids were placed in weighing tins and the weighing tins were then placed in a 100\% humidity equilibrium chamber for 72 hours. Once all the samples were at a water content equilibrium, half of each sample was placed into its own pre-weighed weighing tin and weighed. The weight of the tin, and the wet sample were recorded for each sample. The original wet samples were placed back into the equilibrium chamber to ensure their water content would not change prior to experimentation. The weighed wet samples were placed into the dryer oven at $101^{\circ} \mathrm{C}$ for 72 hours. Once the samples were dry, they were weighed again to determine water content of the original samples still in the equilibrium chamber.

Preparation of the testing vials

Each sample now had a known moisture content. Quantitative saccharification (se below) found the solids to be approximately $63 \%$ cellulose. This was used to then determine the amount of sample needed for each testing vial to achieve a final glucose level of less than $2 \mathrm{~g} / \mathrm{L}$ in the post hydrolysate

Next the pH 5.0 buffer solution with preservative was prepared. This was done by mixing citric acid with DI water and adding benzoic acid to the mixture at $0.49 \%$. The mixture was then titrated with sodium hydroxide $(\mathrm{NaOH})$ until the $\mathrm{pH}$ was raised to 5.0 and remained constant.

Finally, the amount of enzyme mixture was determined. Enzyme loadings were set to 20 cellulase units per gram of solid sample and 100 units of beta-glucosidase per gram of solid sample.

Vials were prepared so that each sample was done in duplicate. Once the buffer solution and solid samples were added to the vials, they were prepared to begin the test. $(29,31,32,33,34)$

\section{Enzymatic hydrolysis tests}

The addition of the enzymes was used as time zero $(\mathrm{T}=0)$. After a sample of each testing vial was taken at time zero the hydrolysis testing began. Immediately after taking the time zero samples the testing vials were placed in the shaker bath at $40^{\circ} \mathrm{C}$. The shaker bath oscillator was set to medium (1/2 way on the dial or number 5$)$. The serum vials were then removed for testing every 2 hours for up to 12 hours. They were then tested at 24 hours, 48 hours and 120 hours.

\section{Analysis of the sample}

The enzymatic hydrolysis samples did not have to be diluted, so the sample taken from each serum vial was placed directly into its corresponding cuvette at each sampling hour. Also, the control that was also used for each enzymatic hydrolysis experiment was a sample that contained buffer, preservative and enzymes but no pretreatment sample.

\section{Quantitative saccharification}

This process was done in accordance with NREL LAP-002. Sulfuric acid $\left(\mathrm{H}_{2} \mathrm{SO}_{4}\right)$ was prepared by adding $99 \% \mathrm{H}_{2} \mathrm{SO}_{4}$ to water to achieve a $72 \% \mathrm{H}_{2} \mathrm{SO}_{4}$. The density of $\mathrm{H}_{2} \mathrm{SO}_{4}$ was close to double that of water so a balance was used to determine the amounts needed. The dried samples from the enzymatic hydrolysis tests were used. Samples of approximately 0.4 
grams were dispensed into separate test tubes. Duplicates of each sample were again created for each experiment. All masses were immediately recorded once they were close to the 0.4 grams and before the effects of moisture in the air could affect the weights. A standard of pure cellulose (Avicel) was also measured in triplicate with each experiment. It was also dried in the dryer oven for 72 hours to ensure no moisture content when weighed. The tubes with sample in them were placed in a tube rack which was further placed on ice. The $72 \%$ $\mathrm{H}_{2} \mathrm{SO}_{4}$ mixture was added to each of the tubes creating an acid to sample ratio of $0.01 \mathrm{ml} / \mathrm{mg}$. Once all tubes had sufficient $\mathrm{H}_{2} \mathrm{SO}_{4}$, they were placed into the reciprocal water bath set at $30^{\circ} \mathrm{C}$ and the shaker mechanism set to half way, or number 5 on the dial. The tubes were immersed in the shaker bath for 2 hours. Every 15 minutes they were stirred with glass stirring rods that stayed in each of the tubes to prevent sample loss or cross contamination. The stirring was key to breaking up clumps and ensuring uniform acid penetration to all the sample. While the tubes were in the bath, a set of serum vials corresponding to each test tube was prepared for the next step of the quantitative saccharification test. The $100 \mathrm{~mL}$ serum vials were placed on ice and water was added to each serum vial. The total water added to each vial was $0.27 \mathrm{~mL} / \mathrm{mg}$ of sample minus $20 \mathrm{ml}$. For example, if sample tube \#3 has $400 \mathrm{mg}$ of sample, then $400 \times 0.27-20=88 \mathrm{ml}$ of water were placed into serum vial \#3. The $20 \mathrm{ml}$ of water subtracted from $0.27 \mathrm{~mL} / \mathrm{mg}$ ratio was used to rinse the contents of the test tubes and stirring rods into the serum vials to ensure complete mass transfer. At the end of the 2 hours, the content of each tube was transferred to their respective serum vials. Each tube and stirring rod was washed with $20 \mathrm{ml}$ of water and again transferred to its respective serum vial. Each serum vial was then capped and sealed. They were taken off the ice and placed in the autoclave for 1 hour. Once the autoclaving was complete and the serum vials were cool enough to be handled, they were analyzed.

\section{Analysis of the Quantitative Saccharification Samples}

The glucose analysis method used for the quantitative saccharification samples was the enzymatioc glucose assay, as was done with the enzymatic hydrolysis samples and yeast inhibition samples.

\section{Results}

Data were analyzed using the DU 500 Spectrophotometer and Infinity Glucose Reagent Assay kit. Two metrics were used, total recovered glucose and reaction speed, after the raw numbers were graphed (Absorbance Units versus time, transposed into Glucose yield versus time).

Figure 7.2, below, shows rapid hydrolysis of all samples pretreated at a similar severity. Note that there is no difference between samples with or without carbonic acid. Also, the graph shows that after 24 hours there was little difference in the yield, even when the testing continued for 120 hours (not shown, but the time it is thought to take for enzymes to achieve about $90 \%$ hydrolysis) there was very little difference in the yield.

Figure 7.3, below, shows that reaction severity played a major role in the rate and yield of enzymatic hydrolysis and that as the reaction severity increased so to did the rate and final yield of the enzymatic hydrolysis throughout the range of severities. Again, however, the presence or absence of carbonic acid appears to have played no distinguishing role in rates or extents of enzymatic hydrolysis.

Figure 7.2 Enzymatic Hydrolysis of Pretreated Aspen Wood 


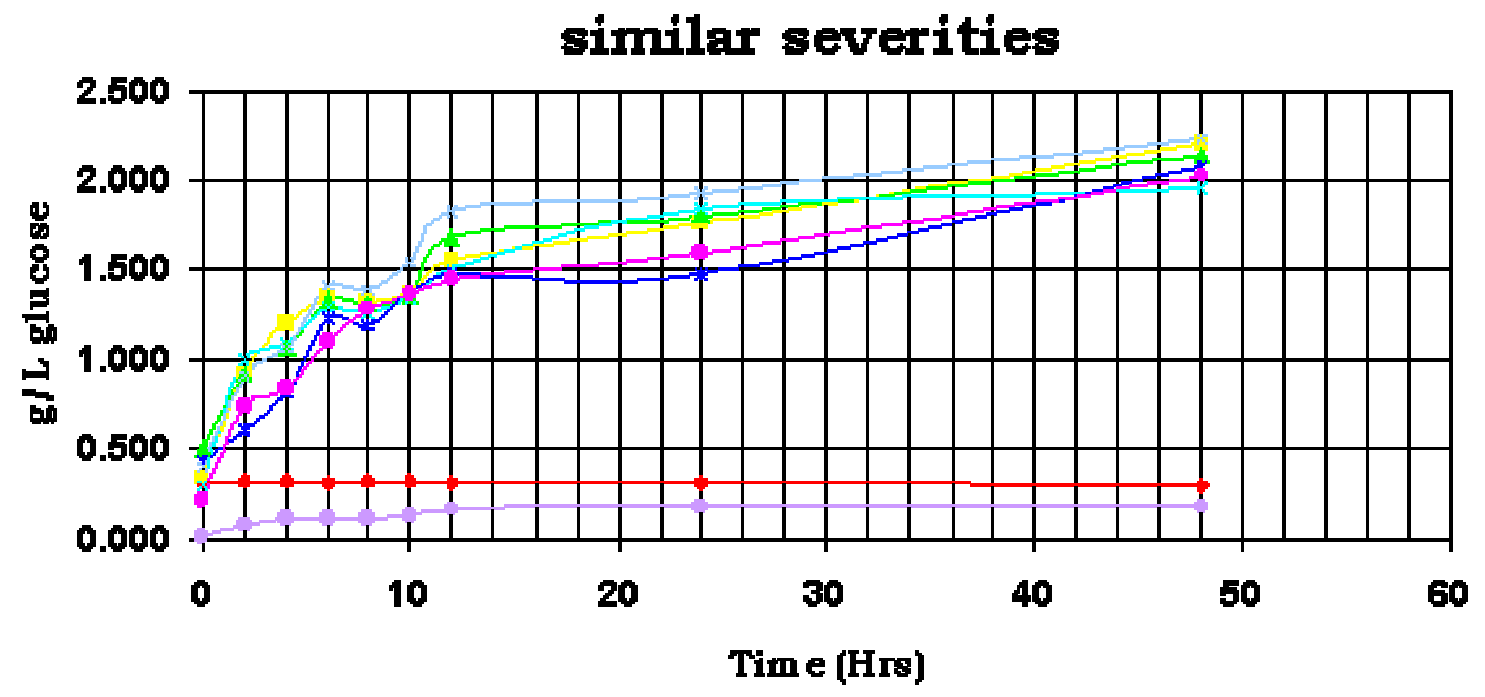

$\begin{array}{|llll|}\rightarrow-\text { standard } 2 & -1=180 / 32 / 0 & -2=180 / 32 / 800 & -3=190 / 16 / 0 \\ \rightarrow-4=190 / 161800 & -5=200 / 8 / 0 & -6=200 / 8 / 800 & -13=\text { buflenz/pres }\end{array}$

Figure 7.3: Enzymatic hydrolysis of Aspen wood pretreated at varying severity.

Param ters at $180 \mathrm{c} / 8,200 \mathrm{C} / 16$, $220 \mathrm{C} / 32$

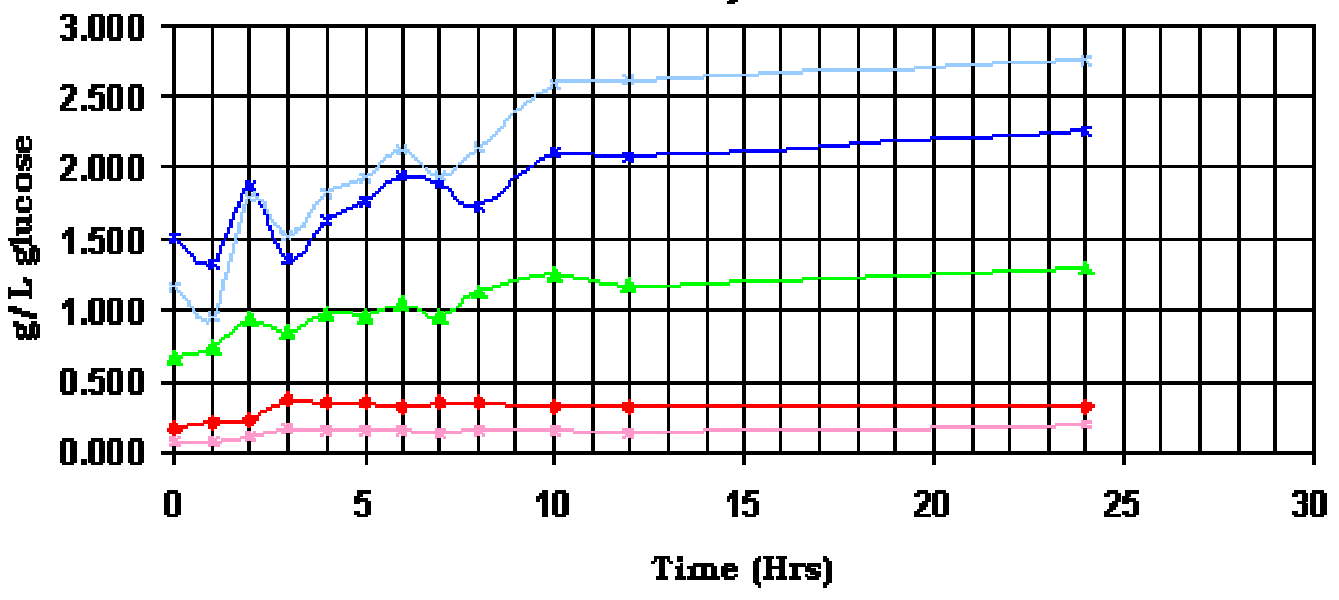

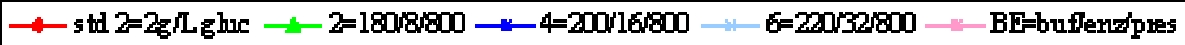

\section{Discussion}

The midpoint pretreatment condition of $200^{\circ} \mathrm{C}$ for 16 minutes does not appear to be the optimum condition for enzymatic hydrolysis. It is clear that the optimal severity of $200^{\circ} \mathrm{C}$ for 
32minutes that produces the maximum xylose sugars as found by McWilliams (2002) from the pretreatment step is not the optimal severity for enzymatic hydrolysis. The rates and yields of enzymatic hydrolysis continued to increase as the reaction severity of the pretreatment increased. It is possible that the enzymatic hydrolysis rates and yields would continue to increase past the maximum severity that was tested of $220^{\circ} \mathrm{C}$ for 32 minutes. A possible explanation for this result is that the more sever reaction conditions are continuing to breakdown the hemicellulose and solubilize the lignin, allowing the enzymes more and easier access to the cellulose

\section{Conclusion}

Pretreatment with carbonic acid showed no significant advantage when compared to pretreatment with liquid hot water. Differences between the samples could be attributed to testing error since in some cases the samples with carbonic acid showed improved enzymatic hydrolysis rates and yields and in other cases they showed a decrease in enzymatic hydrolysis rates and yields.

The reaction severity did have a significant effect on the enzymatic hydrolysis yields of the samples. On average, for every 10 fold increase in severity, the enzymatic hydrolysis yield increased by about 30\%. More testing would be needed to determine the increase in rate. Also, data points need to be tightened and a standard for slope determination amongst the samples would need to be determined.

\section{References}

McWilliams R. C., Van Walsum G. P., 2001, Comparison of Aspen Wood Hydrolysates Produced by Pretreatment with Liquid Hot Water and Carbonic Acid, 3-17

McWilliams, R.C. 2002 Master's Thesis, Comparison of Aspen Wood Hydrolysates Produced by Pretreatment with Liquid Hot Water and Carbonic Acid, p1-17 